\title{
Conformance Test Architecture and Test Suite for ANSI/NIST-ITL 1-2007
}

\author{
Fernando L. Podio \\ Dylan Yaga \\ Christofer J. McGinnis
}

National Institute of Standards and Technology U.S. Department of Commerce 
NISTIR 7791

\section{Conformance Test Architecture and Test Suite for ANSI/NIST-ITL 1-2007}

Fernando L. Podio

Dylan Yaga

Christofer J. McGinnis

June 2011

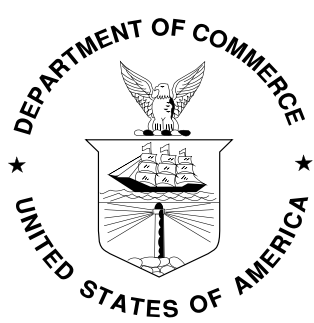

U.S. Department of Commerce Gary Locke, Secretary

National Institute of Standards and Technology Patrick D. Gallagher, Director 


\section{Reports on Computer Systems Technology}

The Information Technology Laboratory (ITL) at the National Institute of Standards and Technology (NIST) promotes the U.S. economy and public welfare by providing technical leadership for the Nation's measurement and standards infrastructure. ITL develops tests, test methods, reference data, proof of concept implementations, and technical analysis to advance the development and productive use of information technology. ITL's responsibilities include the development of technical, physical, administrative, and management standards and guidelines for the cost-effective security and privacy of sensitive unclassified information in Federal computer systems. This Interagency Report discusses ITL's research, guidance, and outreach efforts in computer security, and its collaborative activities with industry, government, and academic organizations.

\section{National Institute of Standards and Technology Interagency Report 76 pages $(2011)$}

Certain commercial entities, equipment, or materials may be identified in this document in order to describe an experimental procedure or concept adequately. Such identification is not intended to imply recommendation

or endorsement by the National Institute of Standards and Technology, nor

is it intended to imply that the entities, materials, or equipment are necessarily the best available for the purpose. 


\begin{abstract}
The Computer Security Division of NIST/ITL supports the development of biometric conformance testing methodology standards and other conformity assessment efforts through active technical participation in the development of these standards and the associated conformance test architectures and test suites. The ANSI/NIST-ITL standard "Data Format for the Interchange of Fingerprint, Facial \& Other Biometric Information" is used by law enforcement, intelligence, military, and homeland security organizations throughout the world. The current version specified in its Traditional Format, is Part 1: ANSI/NIST-ITL 1-2007. Although a revised and augmented version of the standard is under development, the 2007 version is still widely used. The Conformance Test Architecture and Test Suite described in this publication are designed to test implementations of ANSI/NIST ITL 12007. The code (Beta 0.4) is currently designed to support testing of selected record types of the standard but can be extended to support other record types as required. A high-level overview of the architecture and test suite as well as software details and the code structure are provided. A quick start user guide and a comprehensive table of the standard's requirements and the associated implemented conformance test assertions (over five-hundred and thirty) are included.
\end{abstract}

\title{
Disclaimer
}

Statements made in this paper should not be interpreted as standards, guidelines, best practices, or recommendations for specific changes to any other NIST publications.

\section{Acknowledgment}

The authors would like to acknowledge Mark Jerde from ID Technology Partners (NIST contractor for this project) as the principal software developer of the Conformance Test Architecture/Test Suite addressed in this publication.

\section{Sponsor}

The work described in this document was sponsored by The Department of Homeland Security/USVISIT Program.

\section{Feedback}

The Conformance Test Architecture (CTA)/Test Suite (CTS), sample data and documentation can be downloaded from the following web site:

http://www.nist.gov/itl/csd/biometrics/biocta_download.cfm.

Feedback on the CTA/CTS, the sample data and documentation are welcome. Please send comments to csdbiomcta@nist.gov. 


\section{Table of Contents}

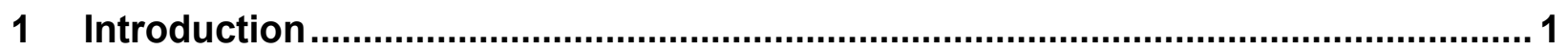

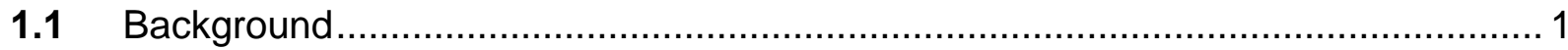

1.2 Need for Conformance Testing to Biometric Standards ....................................... 1

1.3 Support for the ANSI/NIST- ITL 1-2007 Standard ............................................. 2

1.4 Requirements in the Standard and Conformance Test Assertions ........................ 2

1.5 Hierarchy of Conformance Tests ..................................................................... 3

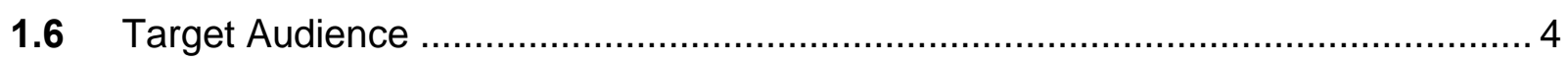

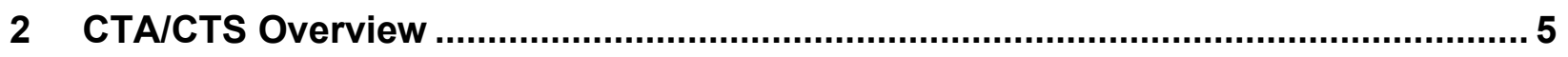

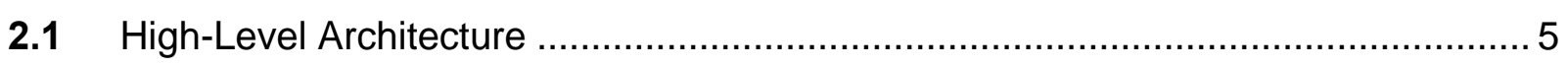

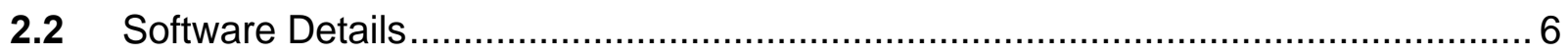

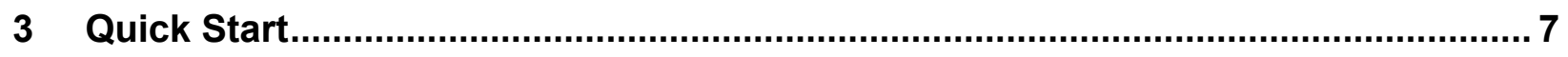

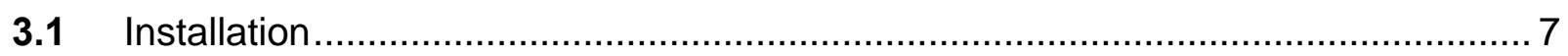

3.2 Loading and Running Implementations ....................................................... 8

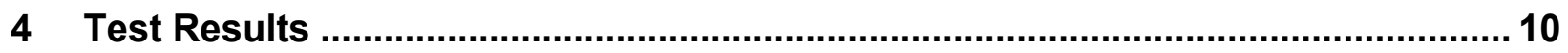

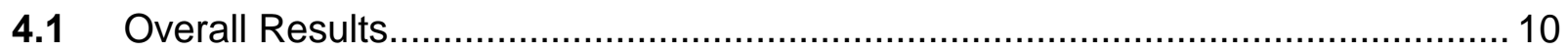

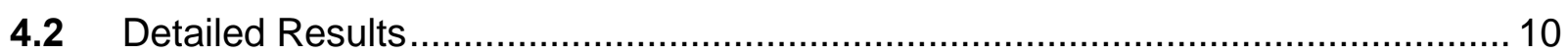

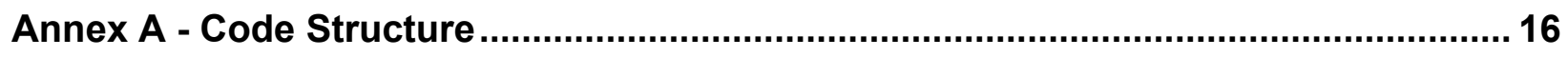

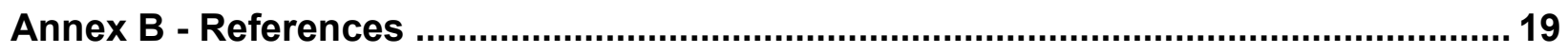

Annex C - Standard Requirements and Conformance Test Assertions ....................... 20

\section{List of Figures and Tables}

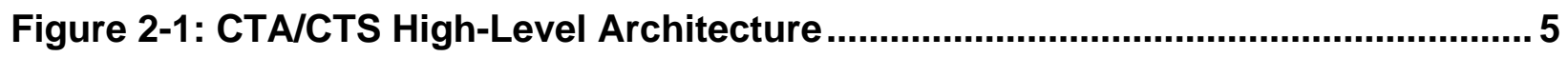

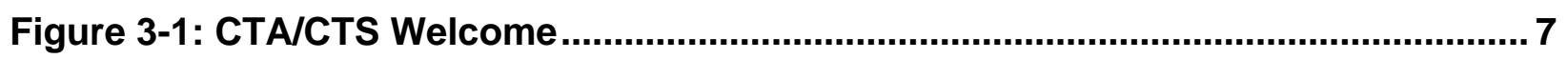



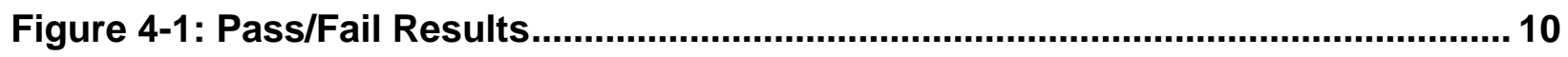

Figure 4-2: ANSI/NIST-ITL 1-2007 Detailed Results ....................................................... 11

Figure 4-3: Output Options ........................................................................................... 11

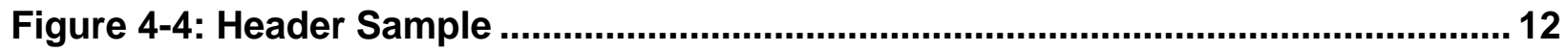

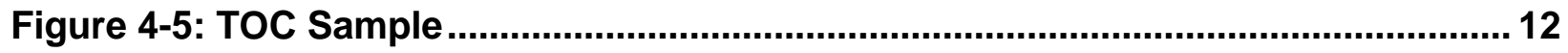

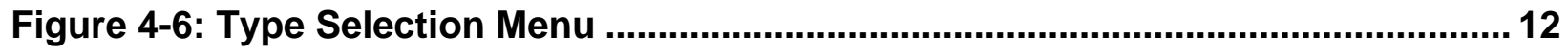




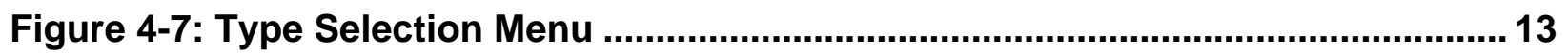

Figure 4-8: Field-Level Parse Test Sample ……..................................................... 13

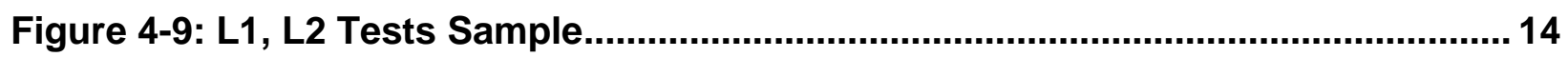

Figure 4-10: File-Level Results Sample................................................................ 14

Figure 4-11: Record-Level Results Sample.............................................................. 14

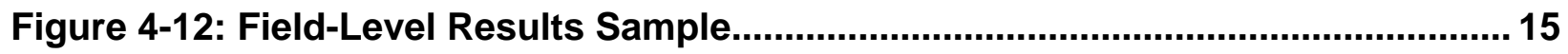

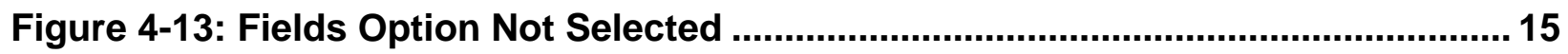

Figure A-1: Class Diagram for the An107 File Class................................................. 17

Figure A-2: Class Diagram for the Data Types ........................................................... 18

Table C-1 - Operators Use in Table C-4 Test Assertions .......................................... 20

Table C-2 - Description of the Operands Used in Table C-4 Test Assertions ...............21

Table C-3 Terms Used in Table C-4 Test Assertions ..................................................... 22

Table C-4 - Requirements and Conformance Test Assertions .................................... 23 


\section{Introduction}

\subsection{Background}

The ANSI/NIST-ITL standard "Data Format for the Interchange of Fingerprint, Facial \& Other Biometric Information" is used by law enforcement, intelligence, military, and homeland security organizations throughout the world. The first version of the standard dates to 1986. Over the years, it has been updated and expanded to cover more biometric modalities beyond the original record type of fingerprint minutiae. Current modalities are:

- Fingerprint minutiae

- Fingerprint image (flat/plain, rolled and latent)

- Scars, marks and tattoos

- Facial image

- Iris image

- Palmprint image

The current version of the ANSI/NIST-ITL standard is:

Part 1 - (ANSI/NIST-ITL 1-2007) in Traditional Format [1]

Part 2 - (ANSI/NIST-ITL 2-2008) in NIEM-conformant XML format [2]

In addition, there is an extension to the table of finger position codes in both parts of the standard to handle multiple-finger captures: ANSI/NIST-ITL 1a-2009 [3].

The standard is now in the process of being revised. A workshop was held July 27-29, 2010 at NIST to determine what new biometric modalities and associated data should be included in a new version of the standard. Working groups established at this workshop provided input for the updated standard. Two drafts were developed (the first draft incorporated the output of the working groups). The second draft incorporated changes based on comments received after the first draft was issued. A second workshop, to discuss this draft was held on March 1-3, 2011 at NIST in Gaithersburg, MD., USA. A third draft was developed after the second workshop. At the time of this writing a new draft (fourth draft) was made available for review and comment. The fourth draft can be downloaded from the ANSI/NIST-ITL Standard Homepage [4].

\subsection{Need for Conformance Testing to Biometric Standards}

The existence of biometric standards alone is not enough to demonstrate that products meet the technical requirements specified in the standards. Conformance testing captures the technical description of a specification and measures whether an implementation faithfully implements the specification. The Computer Security Division (CSD) of NIST/ITL supports the development of biometric conformance testing methodology standards and other conformity assessment efforts through active technical participation in the development of biometric standards and the development of associated conformance test architectures and test suites. NIST/ITL CSD develops these conformance test tools to support users that require conformance to selected biometric standards and to also support product developers interested in conforming to biometric standards by using the same testing tools available to users. These efforts support the possible establishment of conformity 
assessment programs to validate conformance to biometric standards. Conformance testing provides developers, users, and purchasers with increased levels of confidence in product quality and increases the probability of successful interoperability. A Conformance Test Architecture (CTA) as well as Conformance Test Suites (CTS) designed to test implementations of selected biometric data interchange formats developed by Technical Committee M1- Biometrics [5] of the InterNational Committee for Information Technology Standards (INCITS) and Subcommittee 37 (SC 37) Biometrics of the Joint Technical Committee 1 (JTC 1) of ISO and IEC [6] are available for download at a NIST/ITL CSD web page [7].

\subsection{Support for the ANSI/NIST- ITL 1-2007 Standard}

Although a revised and augmented version of the standard is under development, the 2007 version is still widely used. NIST/ITL CSD developed a CTA/CTS (Beta version 0.4) designed to test implementations of selected Record Types of ANSI/NIST-ITL 1-2007, Data Format for the Interchange of Fingerprint, Facial, \& Other Biometric Information - Part 1. These Record Types were considered the first-priority tier. In addition to testing Record Type 1, "Transaction Information Record", the selected records supported by the CTA/CTS are:

- Type 4, High-resolution grayscale fingerprint image

- Type 10, Facial and SMT image

- Type 13, Variable-resolution latent image

- Type 14, Variable-resolution fingerprint image

- $\quad$ Type 17 , Iris image

Over five-hundred and thirty test assertions were implemented. The software code can be extended to support other record types as required. The tool is designed to detail a transaction's level of conformance to the standard. The granularity of the output information in the test results is provided in section 4. The CTA/CTS can also be used as an analysis tool for determining which parts of the transaction (e.g., non-conformant fields, records) do not conform to the standard and to analyze the reason for their nonconformance. For unsupported Record Types only the length of the record is reported during testing, and no determination of conformance is made for these records. In addition to stating whether a file passes or fails, the messages provided include warnings and notes.

\subsection{Requirements in the Standard and Conformance Test Assertions}

The CTA/CTS code implements the assertions required to test all of the mandatory, conditional and optional requirements for Record Type 1 and the other supported Record Types ${ }^{1}$. One or more assertions are required to adequately test conformance for a given requirement. Section 1.5, below, briefly describes Levels 1, 2 and 3 test assertions; more detail is available in the standards identified in the footnote $2^{2}$. In addition to parsing tests which are initially performed, over five-hundred Level

\footnotetext{
${ }^{1}$ Although the standard specifies the Record Type fields as either being Mandatory or Optional, many of the Optional fields are conditional to the content of other fields/requirements.

${ }^{2}$ The concepts addressed in sections 1.4 and 1.5 are based on definitions and discussions included in the following standards: ISO/IEC 29109-1:2009, Conformance testing methodology for biometric data interchange formats defined in ISO/IEC 19794 Biometric Data Format Standard - Part 1: Generalized conformance testing methodology and the $7^{\text {th }}$ draft of Conformance testing methodology for ANSI/NIST- ITL 1- 2011 developed by the ANSI/NIST-ITL Conformance Working Group, February $22,2011$.
} 
1 and 2 assertions were implemented in the code. The assertions also apply to a particular scope, with the scope being "File", "Record" or "Field.". Level 3 assertions were not implemented.

Table C-3 of Appendix C documents the requirements and test assertions for the selected Record Types. Requirements in the standard that are not tested in the current CTA/CTS release version (Beta Version 0.4) are noted in the table. They include: (a) requirements related to International Character Sets; (b) image-based requirements where the image metadata required to implement the assertion is not defined in the image standard; and (c) four assertions related to the alternate finger segment position(s) (ASEG) specified in field 14.025. The required algorithms to implement these assertions are under research.

\subsection{Hierarchy of Conformance Tests}

Three levels of conformance testing are defined below:

\section{Level 1 - Checking internal content}

In Level 1 testing, an ANSI/NIST-ITL 1-2007 transaction(s) is checked for field-by-field, subfieldby-subfield and information item-by information item conformance with the specification of the standard, both in terms of ranges, character types, and cardinality. Since Level 1 testing can be performed by a simple field-by-field, subfield-by-subfield and information item-by information item reading of the standard and comparison to known values, and their encoding, performing this level of conformance testing only requires a transaction or a set of ANSI/NIST transactions (as opposed to a computer algorithm or a set of hardware and software). Therefore, any hardware or software components of the implementation being tested do not have to come into the possession of the testing lab. Only ANSI/NIST-ITL 1-2007 transactions created with those components need to be available.

\section{Level 2 - Internal consistency checking}

In Level 2 testing, an ANSI/NIST-ITL 1-2007 transaction(s) is checked to determine if it is internally consistent. This is achieved by relating values from one or more fields, subfields, or information items within a transaction to other values within the same transaction. Level 2 tests involve interactions between multiple values from different parts of the ANSI/NIST-ITL 1-2007 standard and sometimes from implicit assumptions that are not explicitly stated in the base standard. Thus, Level 2 tests require more complex validation than Level 1. Similar to Level 1 testing, Level 2 conformance testing only requires an ANSI/NIST-ITL 1-2007 transaction(s). To ensure that all (or almost all) possible internal consistency checks are tested, tests are performed whenever possible with a large number of transactions representing as many as possible different structural variants.

\section{Level 3 - Content checking}

A Level 3 test is intended to test whether an ANSI/NIST-ITL 1-2007 transaction under test is a faithful representation of the original biometric data and that it satisfies those requirements of the standard that are not simply a matter of syntax and format. This level of conformance testing for some requirements might be significantly difficult or even impossible to test. 


\subsection{Target Audience}

Although this publication is written for users of the Architecture/Conformance Test Suite, technical details of the architecture, operations, and the code structure are provided for more technicallyinclined readers. 


\section{CTA/CTS Overview}

\subsection{High-Level Architecture}

The high-level architecture implemented for the CTA/CTS can be described using four key components. As depicted in Figure 2-1, each of these components addresses different testing levels. These components are identified as Pre-Parse testing and Field, Record and File-level testing.

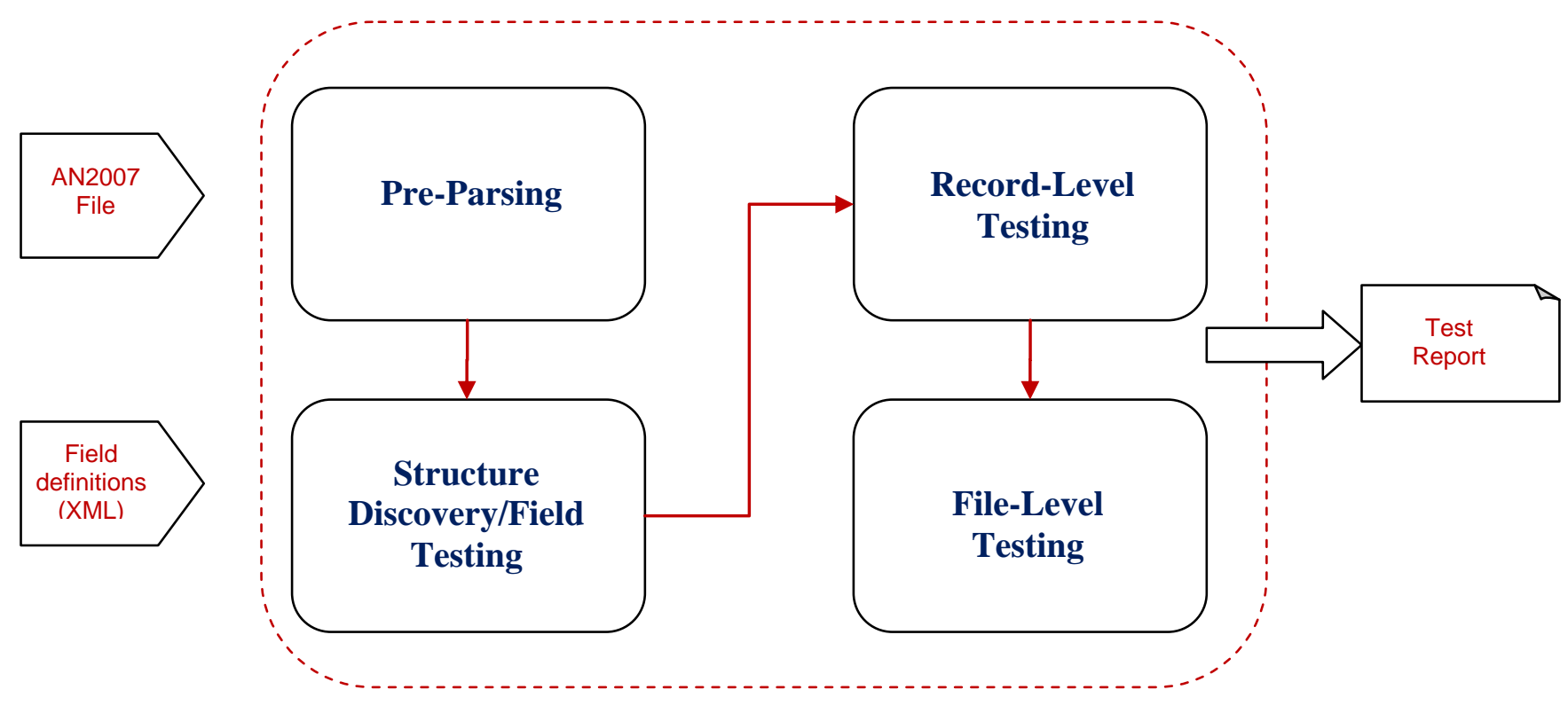

Figure 2-1: CTA/CTS High-Level Architecture

\section{Pre-Parsing Operations}

This operation verifies that the file exists (e.g., it's not NULL) and that the proper arguments are sent to the software. In addition, the file is opened and tests are performed to verify that the first two characters in the file are "1." since every ANSI/NIST-ITL 1-2007 transaction must start with Record Type 1 .

\section{Structure Discovery/Field Testing}

During structure discovery, the Table of Contents (TOC) in Record Type 1 is read and the file is parsed based on the TOC. As the software parses the file, it builds an array containing all fields that are found in each of the records in the transaction. Level 1 testing is then performed for these fields, using the Field definitions (XML file) shown in Figure 2-1. The field definitions file includes metadata on each field such as:

- Type/fields number

- Length min and max

- Min and max occurrences

- Required Level 1 tests 
Level 1 test messages (errors, warning, notes, etc.) are maintained with the corresponding fields within the structure data in memory.

Once this operation is performed and the structure is built, record-level testing is ready to be performed as detailed below. The structure discovery module is designed to allow the parsing method to change based on the type of data present (e.g., binary, ASCII, ASCII/binary). This flexible approach allows also for future revisions of the test tool to allow additional data types to be parsed. The structure discovery/fields testing module includes information on all Level 1 assertions, messages, and message types that will be included in a test report.

\section{Record-Level Testing}

In this module, initial Level 2 tests are performed such as:

- Does the record length equal the number of bytes read?

- Are the correct Record types included in each record?

- For each record, is field 1 first, field 2 second and field 999 last?

Final record Level 2 tests are performed such as checking whether all mandatory and conditional fields are present and whether the field occurrences are correct.

\section{File-Level Testing}

Level 2 tests that include fields of more than one record are performed within this module, such as the requirements associated with the Image Designation Character (IDC) values for each record.

\subsection{Software Details}

The code is developed in C\# under the Microsoft@ .NET 4.0 Framework. The code structure uses interfaces that allow the CTS to treat every Record Type in the same manner while testing. This allows the high-level structure in the code that pertains to all Record Types to be expanded to other Record Types (not supported in this CTA/CTS version) without having redundant code for this level of testing. Derived classes from these interfaces can implement specific tests required for each supported Record Type. Annex A provides a description and class diagram for the code structure, and is oriented towards readers interested in technical details of the code structure. 


\section{Quick Start}

\subsection{Installation}

Run the installation program NIST ITL CSD CTA Beta 0.4.exe, and follow the on screen prompts. The CTA/CTS runs in a Windows environment. It is developed under .NET $\mathrm{v} 4.0$.

After installation, the loaded application should resemble the image below:

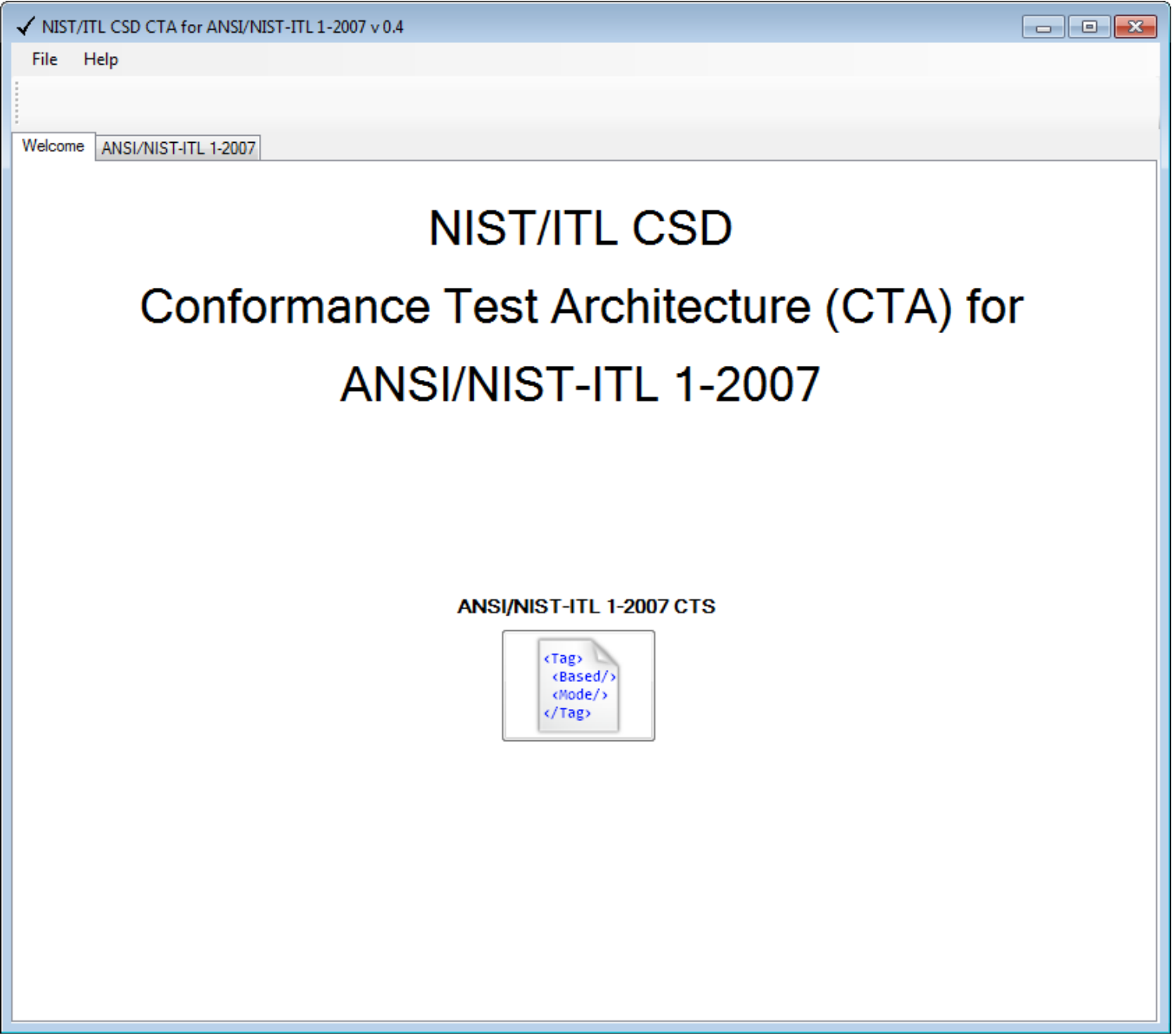

Figure 3-1: CTA/CTS Welcome 


\subsection{Loading and Running Implementations}

The "ANSI/NIST-ITL 1-2007" tab must be selected before files can be loaded. An image of the test window is shown in Figure 3-2.

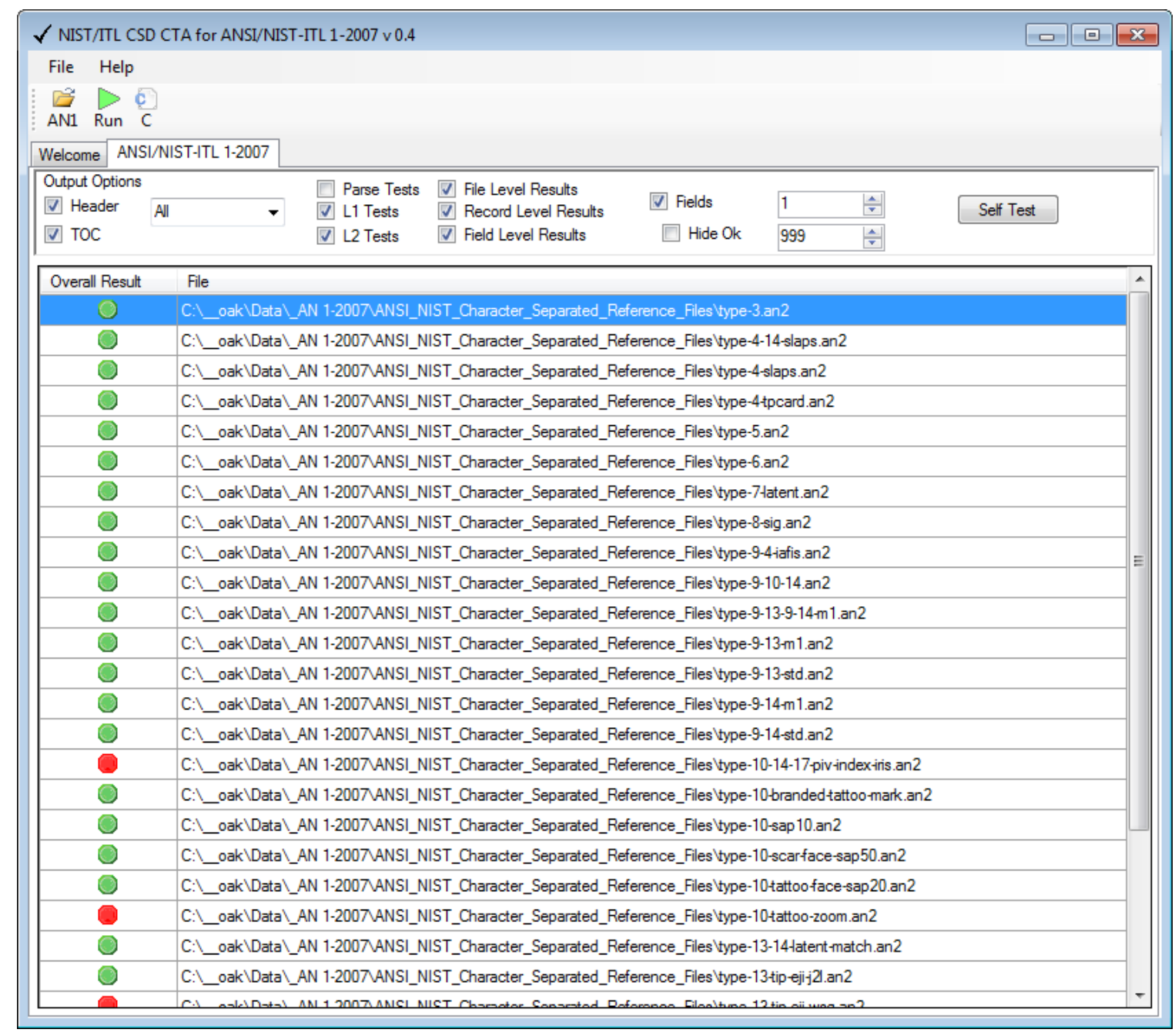

\section{Figure 3-2: ANSI/NIST-ITL 1-2007 Test Window}

\section{Loading Files}

There are two options for loading files:

1. Click the "AN1" button

口

AN1 to specify the file path. If this option is used, the selected files will be loaded but the tests will not run until the "Run" button is selected.

2. Drag and drop one or many files into the window. If this method is chosen, the tests will automatically run and display as passed or failed. 
If necessary, click the "C" (Clear) button $\frac{\mathrm{C}}{\mathrm{C}}$ to remove the loaded files.

\section{Running Tests}

Click the "Run" button Run to run the tests. The files loaded in the window will display as passed or failed, indicated by a green or red circle respectively.

\section{Sample Data Location}

The sample data is available from the following locations for these versions of Microsoft $₫$ Windows ${ }^{\circledR}$ :

- XP: C:IDocuments and Settings\All Users\Documents $\backslash$ ANSI NIST-ITL 1-2007 Sample Files

- Vista: C:IUsers\Public\Documents\ANSI NIST-ITL 1-2007 Sample Files

- 7: C:IUsers\Public\Documents\ANSI NIST-ITL 1-2007 Sample Files 


\section{Test Results}

To generate the test results, specify the file path using the "AN1" button and click the "Run" button to run the tests. Alternatively, drag and drop the files into the GUI and the tests will run automatically.

\subsection{Overall Results}

After running the tests using one of the methods mentioned previously, the overall pass/fail results of the tests will be displayed in the "ANSI/NIST-ITL 1-2007" window. An example of the pass/fail results is shown below, with red (darker) circles representing failures and green circles representing successes.

\begin{tabular}{|c|c|}
\hline Overall Result & File \\
\hline 0 & C:IAN2007/Dataltype-3.an2 \\
\hline 0 & C:IAN2007\Dataltype-4-14-slaps.an2 \\
\hline 0 & C:IAN2007\Data|type-4-slaps.an2 \\
\hline 0 & C:IAN2007/Dataltype-5.an2 \\
\hline 0 & C:IAN2007/Dataltype-6.an2 \\
\hline 0 & C:IAN2007\Dataltype-7-latent.an2 \\
\hline 0 & C:IAN2007\Dataltype-8-sig.an2 \\
\hline O & C:IAN2007/Data|type-9-4-iafis.an2 \\
\hline 0 & C:IAN2007/Dataltype-9-10-14.an2 \\
\hline (1) & C:IAN2007|Data|type-10-14-17-fail.an2 \\
\hline 0 & C:IAN2007/Dataltype-10-branded-tattoo-mark.an2 \\
\hline 0 & C:IAN2007\Data|type-13-tip-eji-j2l.an2 \\
\hline (1) & C:IAN2007|Dataltype-13-tip-eji-wsq-fail.an2 \\
\hline 0 & C:IAN2007|Dataltype-14-amp-nqm-utf8.an2 \\
\hline 0 & C:IAN2007/Dataltype-14-tpcard-nqm.an2 \\
\hline 0 & C:IAN2007/Dataltype-15-palms.an2 \\
\hline (2) & C:IAN2007/Dataltype-17-iris-fail.an2 \\
\hline
\end{tabular}

Figure 4-1: Pass/Fail Results

The complete file path to the implementation is also listed for reference. In addition to these overall results, a detailed summary is also displayed in a separate window. The detailed summary is discussed in the next section.

\subsection{Detailed Results}

The detailed results are displayed in a new window titled "ANSI/NIST-ITL 1-2007", with the tab titled "Full". The contents of this window are defined by the "Output Options" that are selected before running the tests. These options may also be selected after running the tests to update the detailed results dynamically. The image below depicts the detailed summary window with sample results. 


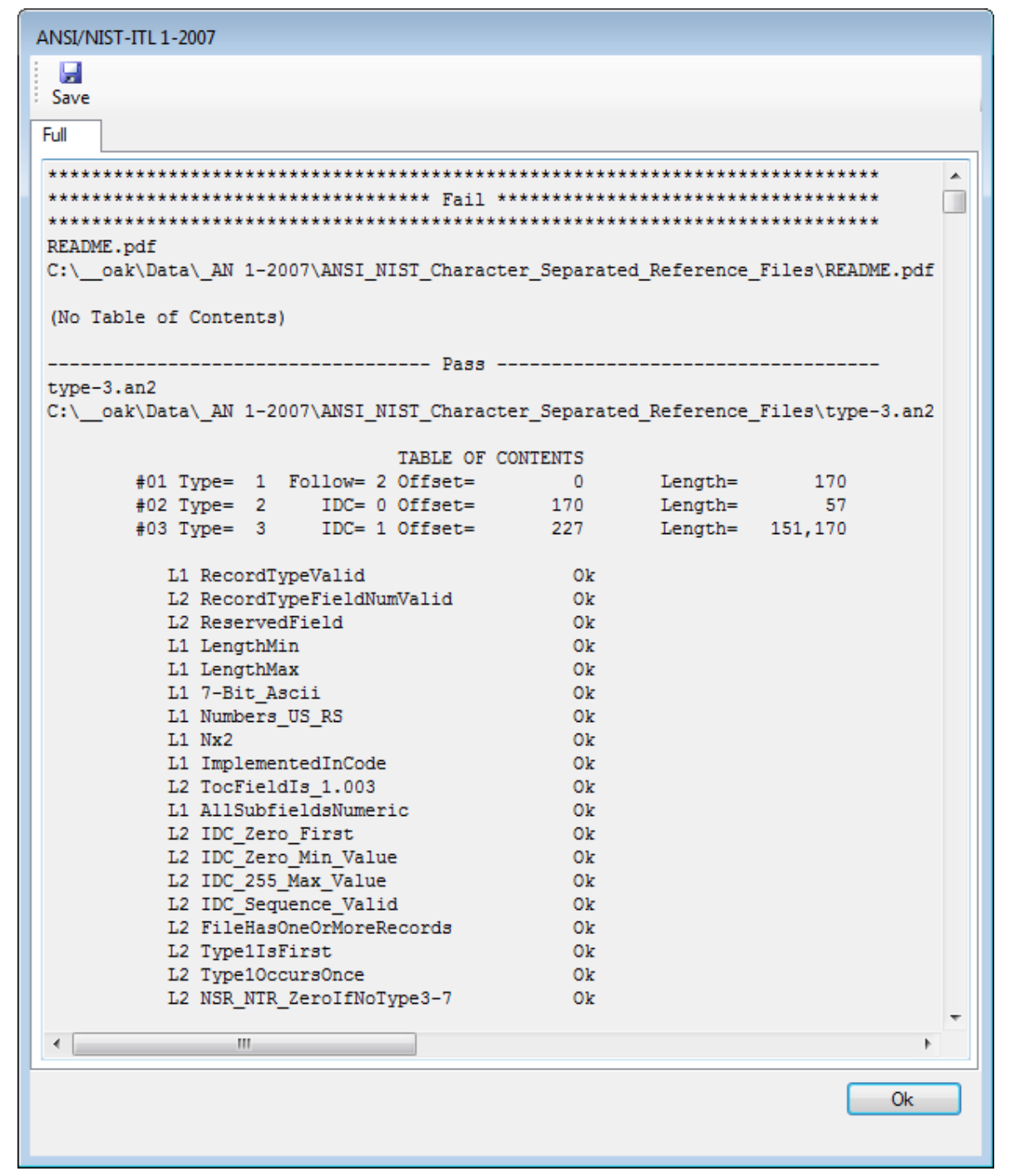

Figure 4-2: ANSI/NIST-ITL 1-2007 Detailed Results

The "Save" button, shown in Figure 4-2 below the "ANSI/NIST-ITL 1-2007" tile bar, may be clicked to save the test results to a text file. After clicking the "Save" button, a Windows Explorer browser window will open to allow a file location and name to be selected.

\section{Output Options}

On the "ANSI/NIST-ITL 1-2007" tab depicted in Figure 3-2, several options are available for formatting the test results detailed output as shown in Figure 4-3 below.

\begin{tabular}{|c|c|c|c|c|c|c|c|}
\hline Output Options & & & $\square$ Parse Tests & $\square$ File Level Results & & 1 & \\
\hline$\checkmark$ Header & All & 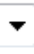 & $\checkmark$ L1 Tests & $\checkmark$ Record Level Results & tields & 1 & $\bar{v}$ \\
\hline$\nabla$ TOC & & & $\checkmark$ L2 Tests & $\checkmark$ Field Level Results & $\square$ Hide Ok & 999 & $\hat{\vec{v}}$ \\
\hline
\end{tabular}

Figure 4-3: Output Options 


\section{- Header}

The "Header" option displays general information about the implementation including the pass/fail status and the file path. A sample header is shown below for a passing implementation.

type-10-branded-tattoo-mark.an2

C: \AN2007\Data \type-10-branded-tattoo-mark.an2

Figure 4-4: Header Sample

\section{- TOC}

The "TOC" option displays a table of contents describing all of the content for a given implementation, including Type, IDC, Offset, and Length. The number preceding each line in the TOC (such as \#01 shown in Figure 4-5) is an identifier assigned to each record found inside of the file as it is sequentially parsed. This identifier clarifies the ordering of record types found within each file.

\begin{tabular}{|c|c|c|c|c|c|c|c|}
\hline & & & & TABLE OF & CONTENTS & & \\
\hline \#01 & Type $=$ & 1 & Follow $=3$ & offset= & 0 & Length= & 185 \\
\hline$\# 02$ & Type $=$ & 2 & $I D C=0$ & Offset= & 185 & Length= & 57 \\
\hline$\# 03$ & Type $=$ & 10 & $I D C=1$ & Offset= & 242 & Length= & 12,373 \\
\hline$\# 04$ & Type= & 10 & $I D C=2$ & Offset $=$ & 12,615 & Length= & 8,016 \\
\hline
\end{tabular}

\section{Figure 4-5: TOC Sample}

\section{- Type Selection}

The type selection window allows the user to select which Record Types should be displayed, including Record Types 1, 4, 10, 13, 14, and 17 (or all record types included in the file). Only the option selected will be displayed in the results. For the unsupported Record Types (any type other than those shown in Figure 4-6), only the name and length of the record will be displayed in the results.

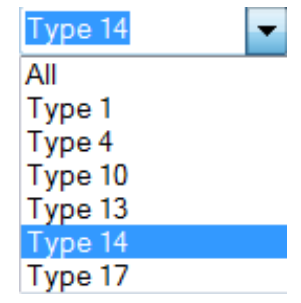

Figure 4-6: Type Selection Menu

For example, the implementation below contains multiple types as shown in the TOC, but only the selected type (14) is shown in the results. 


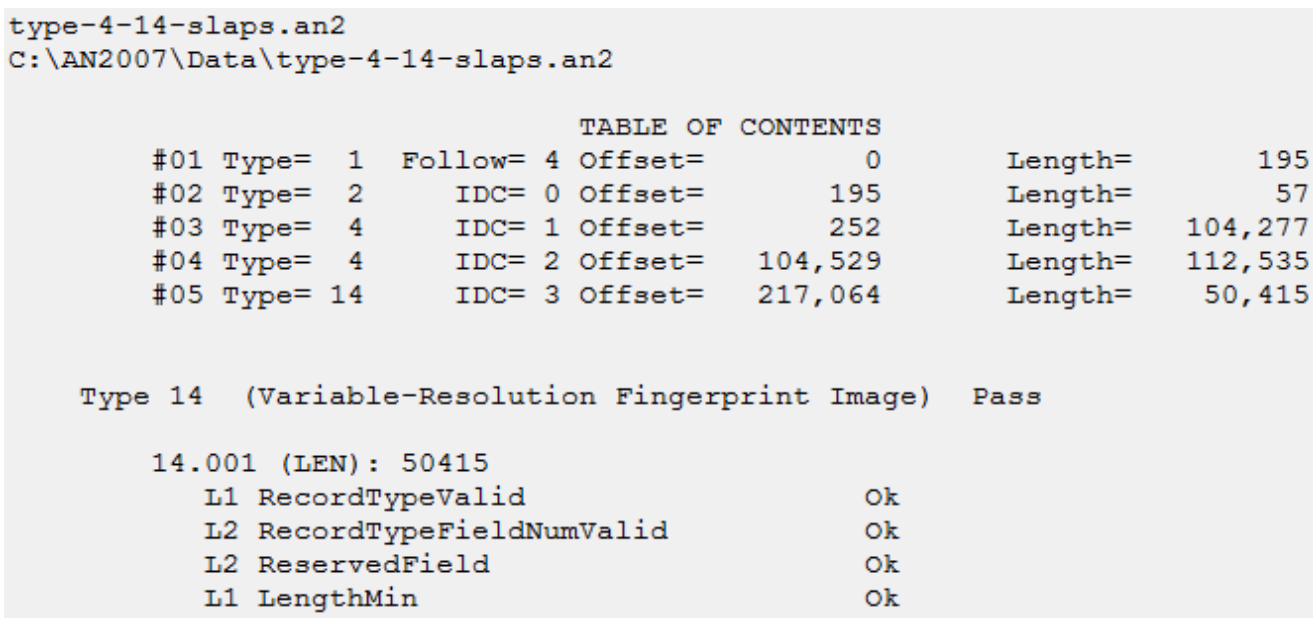

Figure 4-7: Type Selection Menu

\section{- Parse Tests}

The "Parse Tests" option displays parse test results (i.e., tests that are executed during parsing of the file, before the testing of L1 and L2 assertions). The "File Level Results" checkbox must be selected to view the parse tests. See Figure 4-8 for an example of parse tests in the file level results.

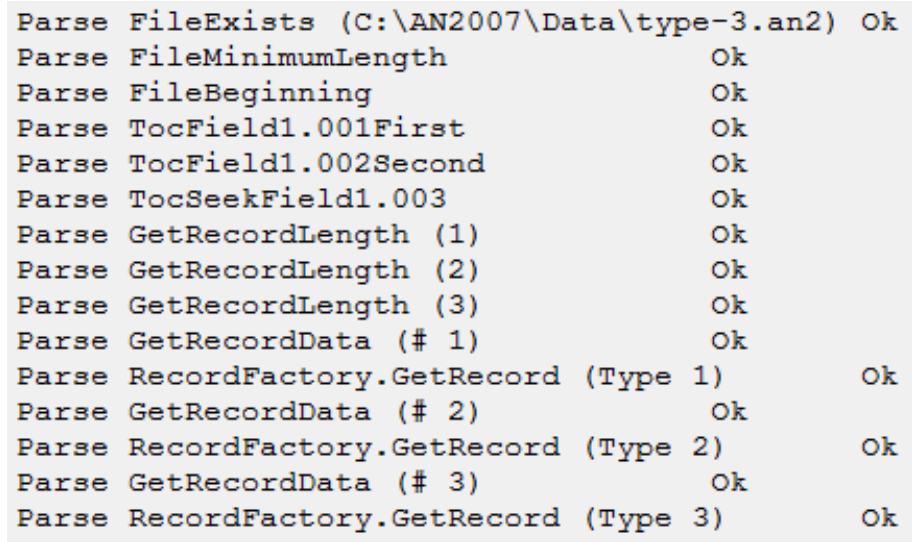

Figure 4-8: Field-Level Parse Test Sample

\section{- L1 Tests/ L2 Tests}

The "L1 Tests" and "L2 Tests" options display L1 and/or L2 test results at the File, Record, and Field levels. The "Fields" checkbox and at least one of the File, Record, or Field Level checkboxes must be selected to view these results. 


\section{- Field Level Results}

The "Field Level Results" option displays all test results for each field. Figure 4-12 depicts a sample of the Field Level Results.
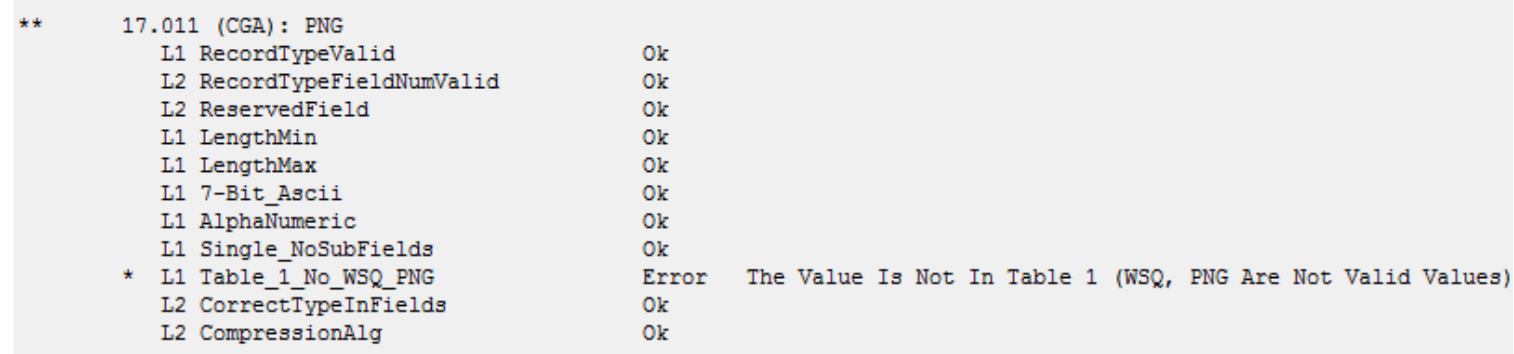

Figure 4-12: Field-Level Results Sample

\section{- Fields}

The "Fields" option displays the fields within each test. If this option is not selected, "Field Level Results" will not be displayed. The image below shows the output when the "Fields" option is not selected, and thus no fields are displayed.



Figure 4-13: Fields Option Not Selected

\section{- Hide Ok}

The "Hide Ok" option removes all tests results that return the status "Ok" from the detailed summary with the exception of Records/Fields in error.

\section{- Field Range}

Specifies which fields of the existing Record Types are displayed by the selected lower and upper bounds in these windows. 


\section{Annex A - Code Structure}

This Annex discusses the elements of the code structure depicted in Figures A-1 and A-2 below.

The An107File Class is the entry point into the CTA/CTS module. It contains a list of all the Record Types encountered in a given transaction (file). After determining that the file exists, the parsing operation begins. As each Record in the file is parsed, it is added to the list of Records within the An107File Class.

IRecordType is an interface that defines required methods and attributes. There is a base Class that derives from this interface called "RecordBase" that performs all common Level 1 and Level 2 assertions for each Record. From RecordBase specific implementations for each Record Type to perform Record Type-specific parsing/testing can be derived. Parsing at this level could, as required, override the general parsing performed at the RecordBase level.

IField is the interface for fields which describes the content of fields in the file under test. IRecordType contains a list of IFields. FieldBase is a specific implementation of IField.

After each test is performed the code updates the list of results at every testing level (File, Record, Field, Subfield, and Subfield data). Possible results, listed in order of increasing severity are: (a) "Ok"; (b) "Note"; (c) "Warning"; (d) "Error"; and "Critical Error". There is also an overall result, once all tests have been completed. A Transaction is determined to be "passing" or "failing" in the OverallResult.

\footnotetext{
${ }^{3}$ The module tries to run all assertions on all applicable data. That is, processing continues after results of Ok, Note, Warning and Error. But the result Critical Error means processing cannot continue because the next valid data element (field or record) could not be parsed successfully.
} 


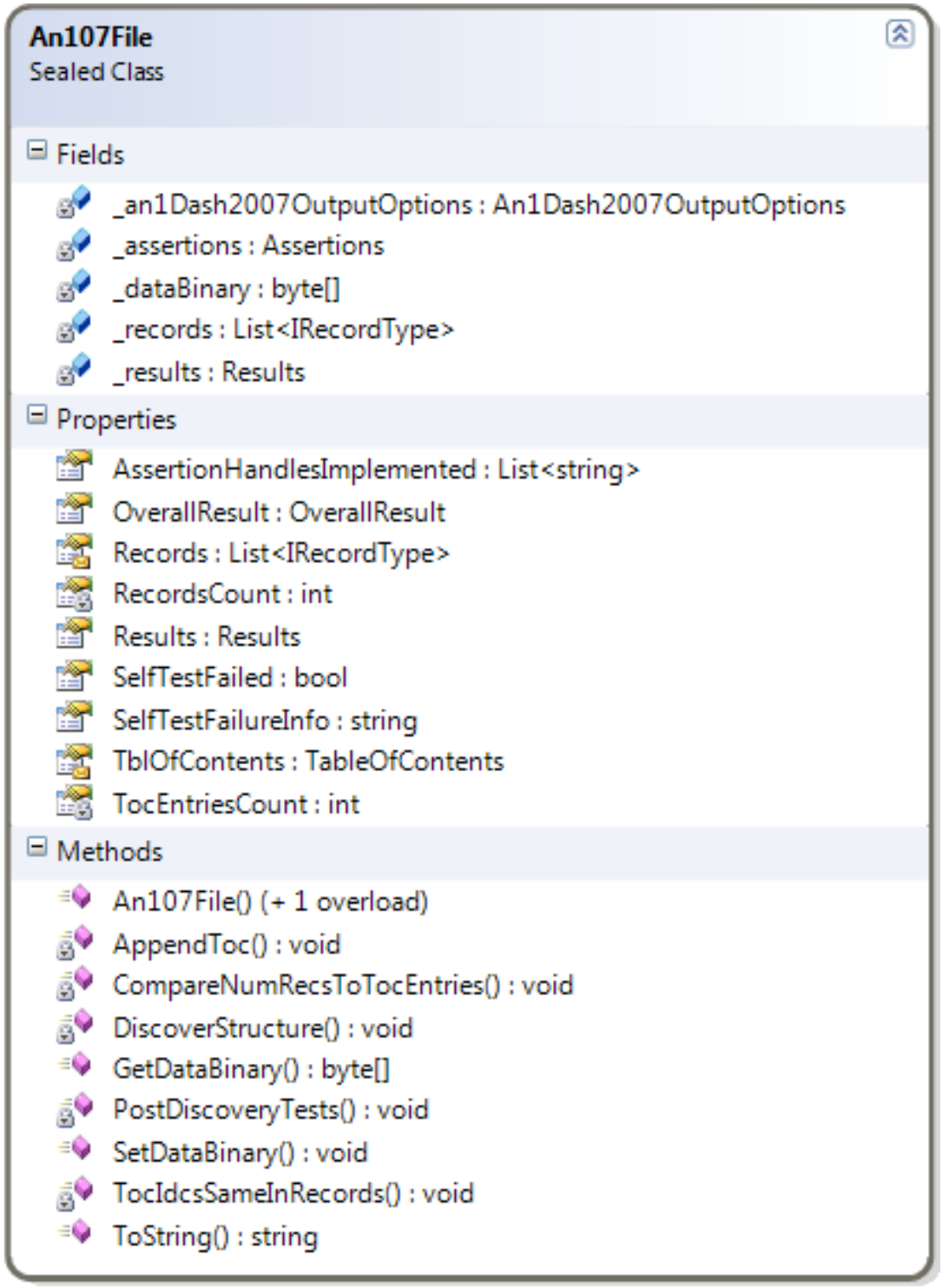

Figure A-1: Class Diagram for the An107 File Class 


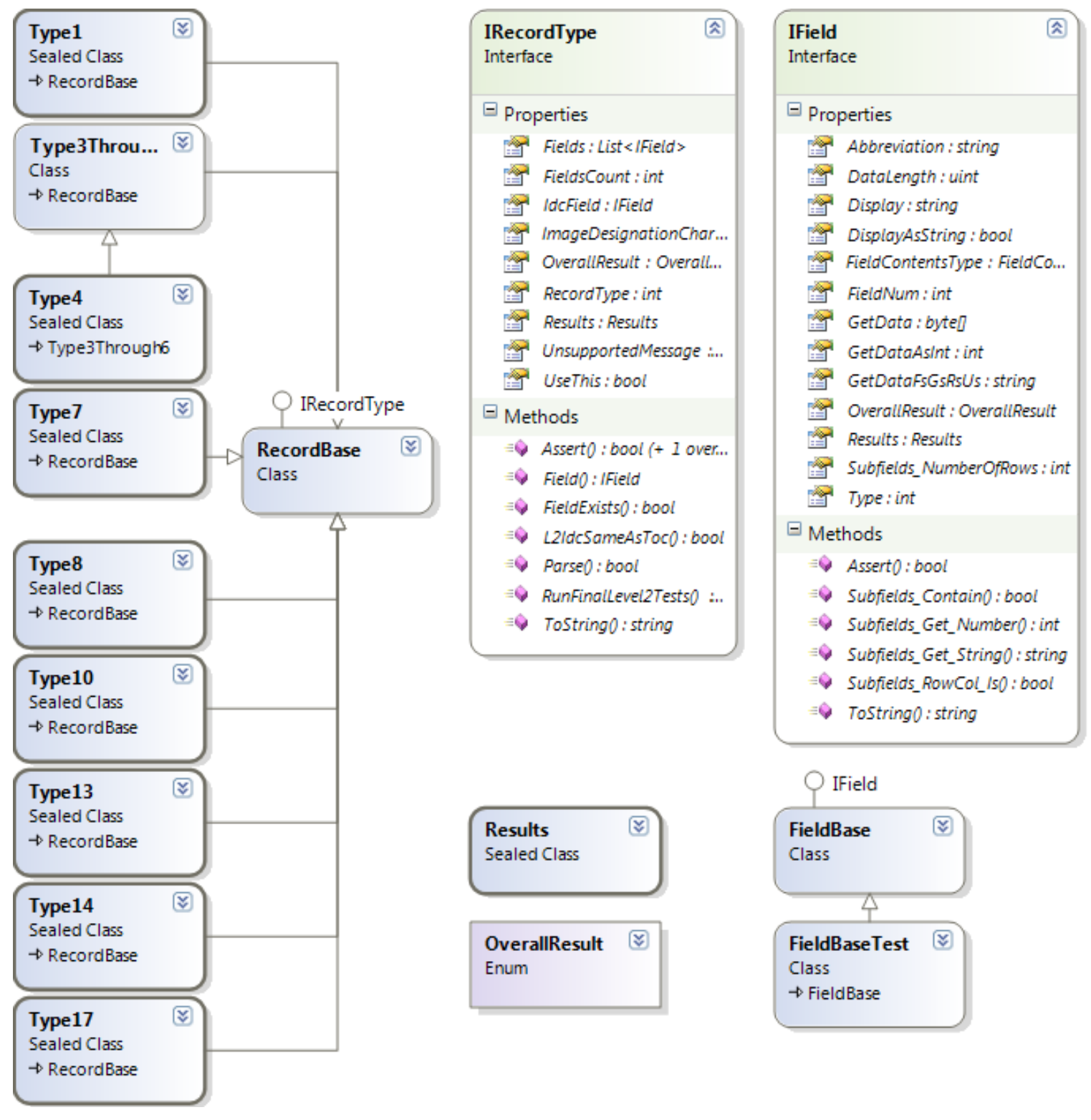

Figure A-2: Class Diagram for the Data Types 
Annex B - References

[1] NIST Special Publication 500-271, ANSI/NIST-ITL 1-2007 (Revision of ANSI/NISTITL 1-2000), American National Standard for Information Systems - Data Format for the Interchange of Fingerprint Facial, \& Other Biometric Information - Part 1

[2] NIST Special Publication 500-275, ANSI/NIST-ITL 2-2008 (XML Version of ANSI/NIST-ITL 1-2007), American National Standard for Information Systems — Data Format for the Interchange of Fingerprint Facial, \& Other Biometric Information - Part 2: XML Version

[3] Update to "Data Format for the Interchange of Fingerprint, Facial \& Other Biometric Information" for multiple finger capture designations ANSI/NIST ITL 1a-2009

[4] ANSI/NIST Standard Homepage - http://www.nist.gov/itl/iad/ig/ansi_standard.cfm

[5] INCITS M1 - Biometrics Public Homepage http://standards.incits.org/a/public/group/m1

[6] ISO/IEC JTC1/SC 37 - Biometrics Public Homepage http://www.iso.org/iso/jtc1_sc37_home

[7] NIST/ITL CSD Biometric Conformance Test Architectures and Test Suites Downloads Web Page: http://www.nist.gov/itl/csd/biometrics/biocta_download.cfm 


\section{Annex C - Standard Requirements and Conformance Test Assertions}

Test assertions documented in Table C-4 below are expressed according to the operators and operands included in Tables C-1 and C-2 respectively, except for those instances where the assertion cannot be clearly or easily represented in a mathematical format. Table C- 3 includes terms used throughout the assertions table. In the tool implementation all image-related assertions associated with compressed image types are tested against the image metadata only and not against the image itself. Even though Logical Record types 3 to 6 are not ASCII data, and are not represented in implementations as a tagged-field (e.g. 4.001), they are represented as such in this table to make it easier to understand. For assertions formulated from several sections of the AN Standard, the assertion is listed once on the first requirement encountered.

\section{Table C-1 - Operators Use in Table C-4 Test Assertions}

\begin{tabular}{|c|l|}
\hline Operator & Description \\
\hline EQ & Equal. Tests for equality between two values. \\
\hline NEQ & Not Equal. Tests for non-equality between two values. \\
\hline GTE & Greater Than or Equal To. Tests if the first value is greater than or equal to the second value. \\
\hline LTE & Less Than or Equal To. Tests if the first value is less than or equal to the second value. \\
\hline GT & Greater Than. Tests if the first value is greater than the second value. \\
\hline LT & Less Than. Tests if the first value is less than the second value. \\
\hline MO & Member Of. Tests if the value is a contained within the set. \\
\hline AND & Logical And. Tests if both values are true. \\
\hline OR & Logical OR. Tests if any values are true. \\
\hline NOT & Negate. Negates any operator or calculation that follows. \\
\hline ST & Such That. For example, $(X, Y)$ ST X GTE 0 AND Y LTE 10 \\
\hline IF & Logical IF. Tests if the condition is true. \\
\hline IFF & IF and Only IF. Tests that the condition guarantees another. \\
\hline MOD & The modulo operator. X MOD Y = the remainder of a X divided by Y \\
\hline$\#$ & All. Represents all legal values. \\
\hline : & Data Element Selector. X:N selects the Nth data element in X. \\
\hline in & The selector operator. X in Y selects only those X found in Y. \\
\hline to & Range Selector. X to Y provides set of values Z ST Z GTE X AND Z LTE Y \\
\hline , & Range concatenation. X,Y provides the set of values X AND Y. \\
\hline
\end{tabular}


Table C-2 - Description of the Operands Used in Table C-4 Test Assertions

\begin{tabular}{|c|c|}
\hline Operand & Description \\
\hline Present(Value) & Determines if Value is present. \\
\hline Present(X in Y) & Determines if X is present in Y. \\
\hline Sum(Values) & The summation of Values. \\
\hline Count(Value) & The number of occurrences of Value. \\
\hline Count(X in Y) & The number of X found in Y. \\
\hline Bytes(Value) & Value of X. \\
\hline MinOccurrences(Value) & All of the byte data contained within Value. \\
\hline MaxOccurrences(Value) & The minimum number of occurrences allowed. \\
\hline MinSize(Value) & The maximum number of occurrences allowed. \\
\hline MaxSize(Value) & The minimum size allowed per occurrence. \\
\hline Min(Value)= & The maximum size allowed per occurrence. \\
\hline Length(Value) & The minimum of the set represented by Value. \\
\hline First(X in Y) & The length of the Value. \\
\hline Second(X in Y) & The first X found in Y. \\
\hline Last(X in Y) & The second X found in Y. \\
\hline Type(Value) & The last X found in Y. \\
\hline FieldNumber(Value) & The type of Record represented by Value. \\
\hline ForEach(X in Y) $\{$ Expression(s) $\}$ & The value of the field number for the field represented by Value. \\
\hline Valid(X) & Evaluate Expression for every X in Y. \\
\hline
\end{tabular}

Note: When a data range is used in a calculation, the calculation is applied independently for each data element in the range. As an example, the expression:

Count(Subfields in 1.001,1.002) EQ 0

is equivalent to:

Count(Subfields in 1.001) EQ 0 AND Count(Subfields in 1.002) EQ 0 
Table C-3 Terms Used in Table C-4 Test Assertions

\begin{tabular}{|c|c|}
\hline Term & Description \\
\hline NULL & Control character with no value. \\
\hline NA & Not Applicable \\
\hline
\end{tabular}

Table C-4 includes the requirements (or a summary of the requirements) specified in ANSI/NIST-ITL 1-2007 standard and associated required conformance test assertions. Although references are included for all Record Types when appropriate (for example when a requirement detailed for "tagged-filed records"), the table focuses on requirements and assertions for the Record Types of interest: Record Type 1 and Record Types 4, 10, 13, 14, and 17. Explanations of the heading for each column in the table are included below.

Table C-4 can be used for conformance test tool developers, implementers of the standard, and end-users as well as testing laboratories File, Record and Field-level requirements and assertions are included.

The table includes the following headings ${ }^{4}$ :

- Requirement ID: Defines a unique identifier for the requirement and associated assertion or set of assertions. It provides reference to the type of requirement (e.g., file, record, and field). It could refer to more than one Record Type.

- Reference in the standard: Identifies the clause where the requirement is included in the standard. In some cases the reference includes additional information such as a Table number.

- Requirement Summary: Provides a summary of the requirement detailed as textual information or an interpretation of the requirement in the standard. It carries the essentials of the requirement but may not provide all the text necessary to understand it.

- Level: Indicates whether Level 1 or Level 2 conformance testing is required to address the requirement in the previous column. Assertions for Level 3 conformance test are not included. A single cell is included for each assertion.

- Status: Reflects the status specified in the standard (Mandatory $(\mathrm{M})$ or Optional $(\mathrm{O})$ ) for each field. It might also indicate whether the requirement in the standard (e.g., field content, presence of field) is considered Mandatory or Optional. Conditional requirements (e.g., conditional to other information items) are tested according to the requirements but are not indicated as such in this column.

- Assertion ID: Defines an identifier of a specific test assertion within the set of test assertions associated with a requirement.

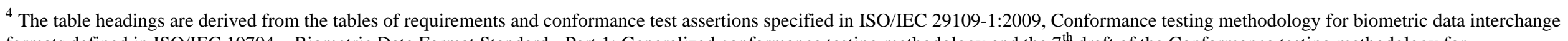

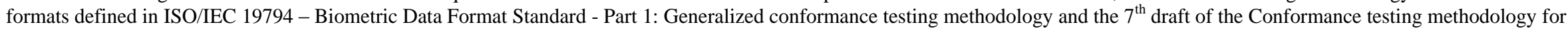
ANSI/NIST- ITL 1-2011 developed by the ANSI/NIST-ITL Conformance Working Group, February 22, 2011. 
- Test assertion: Provides, whenever possible, a mathematical equation or a procedure. Plain text is included whenever a mathematical formula or simple procedure cannot be detailed.

- Test Note: Contains a number of a note included below the table. The test note may include a test procedure when the complexity of the test assertion does not allow this procedure to be included in the test assertion column. It could also include an explanatory note related to the assertion's implementation.

- Implementation support: Denotes a supplier's implementation support of a particular requirement ("Y"/"N"). A note can follow the table when providing more details of implementation support (or the lack of it) is required.

- Supported Range: Indicates a range of values supported, especially when it is different than the full range of values specified in the standard. When an information item is specified as a single value, or does not address a range of values, a N/A should be used.

- Test Result: This column is used to denote the test results. For file and record-level results the results are either "Pass" or "Fail". The field-level results should be indicated as "Ok" "Error", "Warning" and "Note". Explanatory notes can be added below the table.

Table C-4 - Requirements and Conformance Test Assertions

\begin{tabular}{|c|c|c|c|c|c|c|c|c|c|c|}
\hline $\begin{array}{l}\text { Requirement } \\
\text { ID }\end{array}$ & $\begin{array}{c}\text { Reference in } \\
\text { Base } \\
\text { Standard }\end{array}$ & $\begin{array}{l}\text { Requirement } \\
\text { Summary }\end{array}$ & Level & Status & $\begin{array}{l}\text { Assertion } \\
\text { ID }\end{array}$ & $\begin{array}{c}\text { Test } \\
\text { Assertion }\end{array}$ & $\begin{array}{l}\text { Test } \\
\text { Note }\end{array}$ & $\begin{array}{l}\text { Implemen- } \\
\text { tation } \\
\text { Support }\end{array}$ & $\begin{array}{c}\text { Suppor- } \\
\text { ted } \\
\text { Range }\end{array}$ & $\begin{array}{c}\text { Test } \\
\text { Result }\end{array}$ \\
\hline \multicolumn{11}{|c|}{ File-Level Requirements and Assertions } \\
\hline \multirow{10}{*}{ 7_Bit_ASCII } & \multirow{10}{*}{$\begin{array}{c}7, \\
\text { Table } 4 \\
7.1\end{array}$} & \multirow{10}{*}{$\begin{array}{l}\text { The standard defines logical records, } \\
\text { some of which include ASCII tagged } \\
\text { textual fields. The data in the Type-1 } \\
\text { record shall always be recorded in } \\
\text { variable length fields using 7-bit ASCII. } \\
\text { Text data in the Type-2, Type-9 and } \\
\text { tagged-field records will normally use 7- } \\
\text { bit ASCII encoding. Mechanisms for } \\
\text { using character sets other than 7-bit } \\
\text { ASCII are given in section 8.2.3, } \\
\text { International Character Sets. }\end{array}$} & 1 & M & $\begin{array}{c}\text { 7-Bit_Ascii::10.001 } \\
\text { to } 10.013\end{array}$ & $\{10.001$ to 10.013$\}$ LT 128 & & & & \\
\hline & & & 1 & M & $\begin{array}{c}\text { 7-Bit_Ascii::10.016 } \\
10.017\end{array}$ & $\{10.016,10.017\}$ LT 128 & & & & \\
\hline & & & 1 & M & $\begin{array}{c}\text { 7-Bit_Ascii::10.020 } \\
\text { to } 10.030\end{array}$ & $\{10.020$ to 10.030$\}$ LT 128 & & & & \\
\hline & & & 1 & M & $\begin{array}{c}\text { 7-Bit_Ascii::10.040 } \\
\text { to } 10.043\end{array}$ & $\{10.040$ to 10.043$\}$ LT 128 & & & & \\
\hline & & & 1 & M & $\begin{array}{c}\text { 7-Bit_Ascii::13.001 } \\
\text { to } 13.017\end{array}$ & $\{13.001$ to 13.017$\}$ LT 128 & & & & \\
\hline & & & 1 & M & $\begin{array}{c}\text { 7-Bit_Ascii::13.020 } \\
13.024\end{array}$ & $\{13.020,13.024\}$ LT 128 & & & & \\
\hline & & & 1 & M & $\begin{array}{c}\text { 7-Bit_Ascii::14.001 } \\
\text { to } 14.018\end{array}$ & $\{14.001$ to 14.018$\}$ LT 128 & & & & \\
\hline & & & 1 & M & $\begin{array}{c}\text { 7-Bit_Ascii::14.020 } \\
\text { to } 14.025\end{array}$ & $\{14.020$ to 14.025$\}$ LT 128 & & & & \\
\hline & & & 1 & M & 7-Bit_Ascii::14.030 & $\{14.030\}$ LT 128 & & & & \\
\hline & & & 1 & M & 7-Bit_Ascii::17.001 & $\{17.001$ to 17.026$\}$ LT 128 & & & & \\
\hline
\end{tabular}




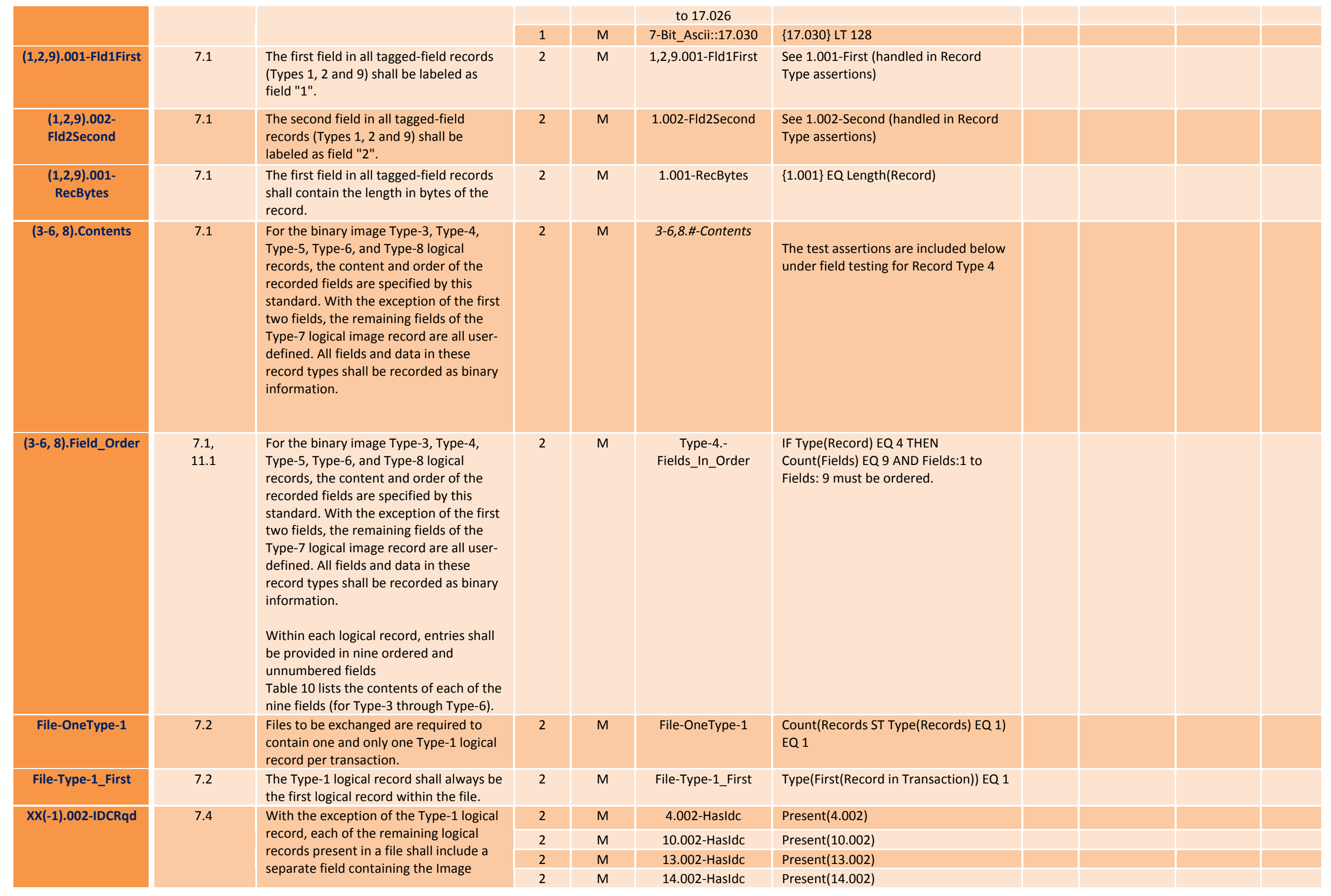




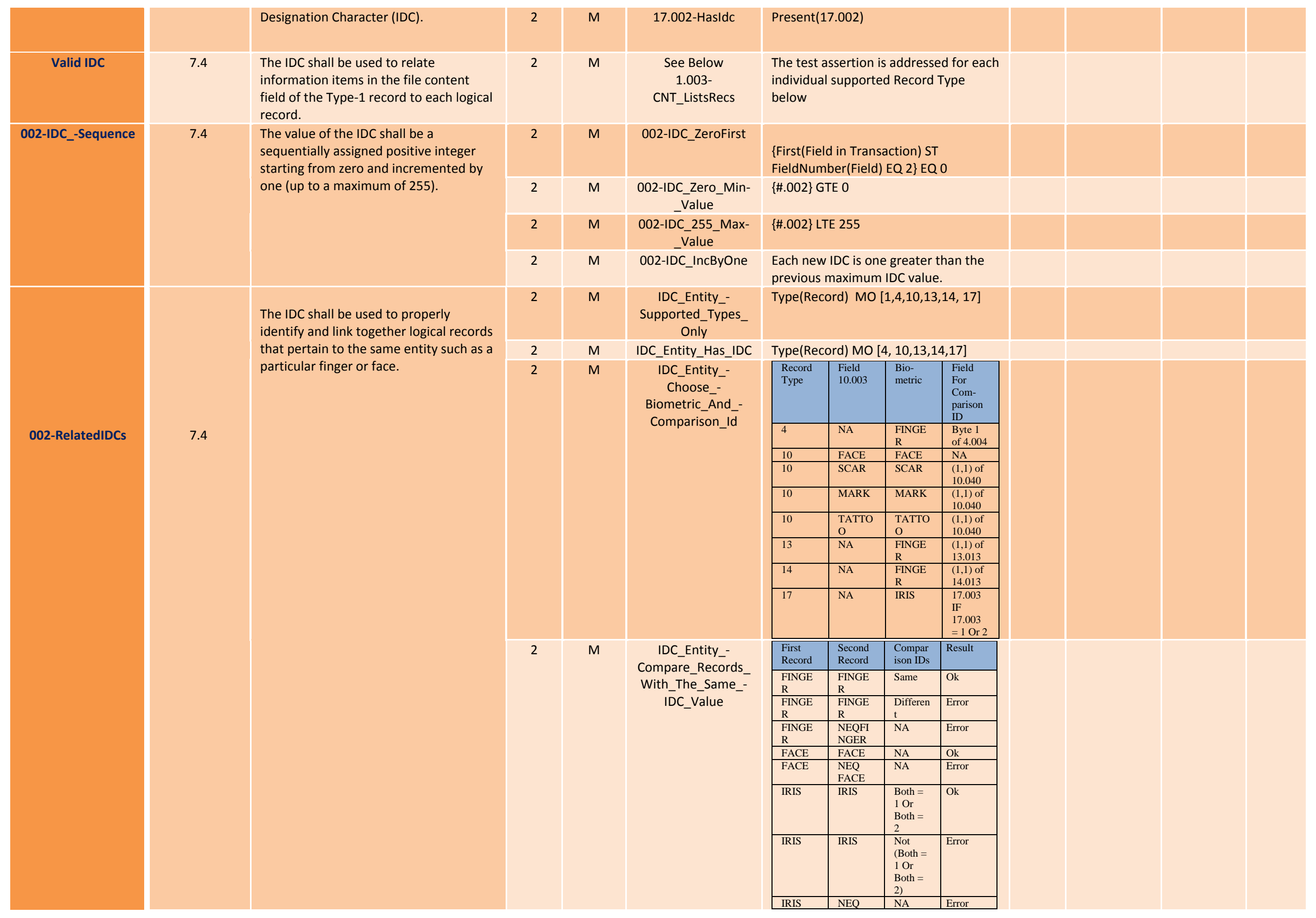



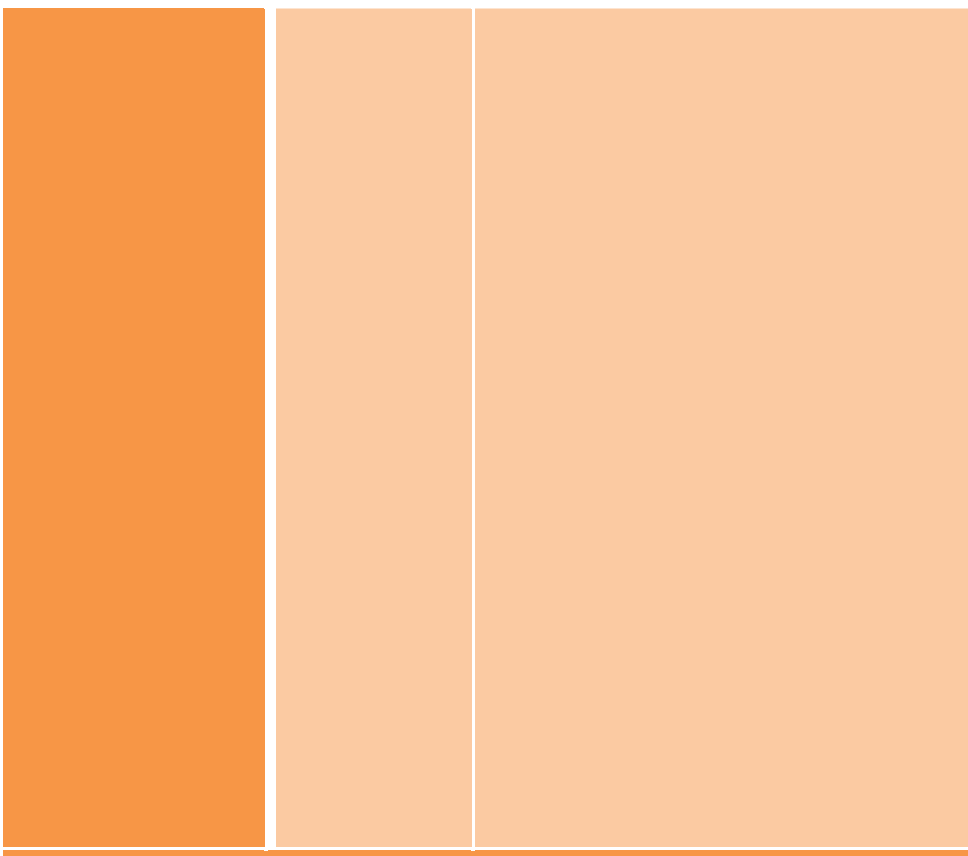

\begin{tabular}{|c|c|c|c|}
\hline & IRIS & & \\
\hline SCAR & SCAR & Same & $\mathrm{Ok}$ \\
\hline SCAR & SCAR & Differen & Warning \\
\hline SCAR & $\begin{array}{l}\text { MARK } \\
\text { Or } \\
\text { TATTO } \\
\text { O }\end{array}$ & $\begin{array}{l}\text { Same or } \\
\text { Differen } \\
t\end{array}$ & \begin{tabular}{|l|} 
Warning \\
\end{tabular} \\
\hline SCAR & $\begin{array}{l}\text { IINGE } \\
\text { R, } \\
\text { FACE } \\
\text { Or IRIS }\end{array}$ & NA & Error \\
\hline MARK & MARK & Same & Ok \\
\hline MARK & MARK & $\begin{array}{l}\text { Differen } \\
t\end{array}$ & Warning \\
\hline MARK & $\begin{array}{l}\text { SCAR } \\
\text { Or } \\
\text { TATTO } \\
\text { O }\end{array}$ & $\begin{array}{l}\text { Same or } \\
\text { Differen } \\
t\end{array}$ & Warning \\
\hline MARK & $\begin{array}{l}\text { FINGE } \\
\text { R, } \\
\text { FACE } \\
\text { Or IRIS }\end{array}$ & NA & Error \\
\hline $\begin{array}{l}\text { TATTO } \\
\text { TaT } \\
\end{array}$ & $\begin{array}{l}\text { TATTO } \\
\mathrm{O}\end{array}$ & Same & Ok \\
\hline $\begin{array}{l}\text { TATTO } \\
\mathrm{O}\end{array}$ & $\begin{array}{l}\text { TATTO } \\
\text { O }\end{array}$ & $\begin{array}{l}\text { Differen } \\
t\end{array}$ & Warning \\
\hline $\begin{array}{l}\text { TATTO } \\
\text { O }\end{array}$ & $\begin{array}{l}\text { SCAR } \\
\text { Or } \\
\text { MARK }\end{array}$ & $\begin{array}{l}\text { Same or } \\
\text { Differen } \\
t\end{array}$ & \begin{tabular}{|l|} 
Warning \\
\end{tabular} \\
\hline $\begin{array}{l}\text { TATTO } \\
\text { O }\end{array}$ & $\begin{array}{l}\text { INNGE } \\
\text { R, } \\
\text { FACE } \\
\text { Or IRIS }\end{array}$ & $\mathrm{NA}$ & \begin{tabular}{|l} 
Error \\
\end{tabular} \\
\hline
\end{tabular}

\section{Record-Level Requirements and Assertions}

\begin{tabular}{|c|}
\hline $\begin{array}{c}\text { File::OneOrMoreRe } \\
\text { cs }\end{array}$ \\
\hline $\begin{array}{c}\text { AppropriateFields- } \\
\text { PerRecord }\end{array}$ \\
\hline No_Subfields \\
\hline
\end{tabular}

A transaction file shall consist of one or more logical records.

For each logical record contained in the

file, several information fields

appropriate to that record type shall be present.

8.2, Sections 8.2 and 8.2.1 describe the 8.2.1 mechanism that enables a field to Various sub- contain multiple information items sections of known as subfields. The following fields 9.1, are defined to have a single information 15.1 , field and contain no subfields:

18.1 , 19.1 22.2

$1.001,1.002,1.004$ to $1.012,1.014$

10.001 to $10.013,10.016,10.017$, $10.020,10.021,10.027,10.030$

13.001 to $13.012,13.016,13.017$ 13.020

14.001 to $14.012,14.016,14.017$ $14.020,14.030$

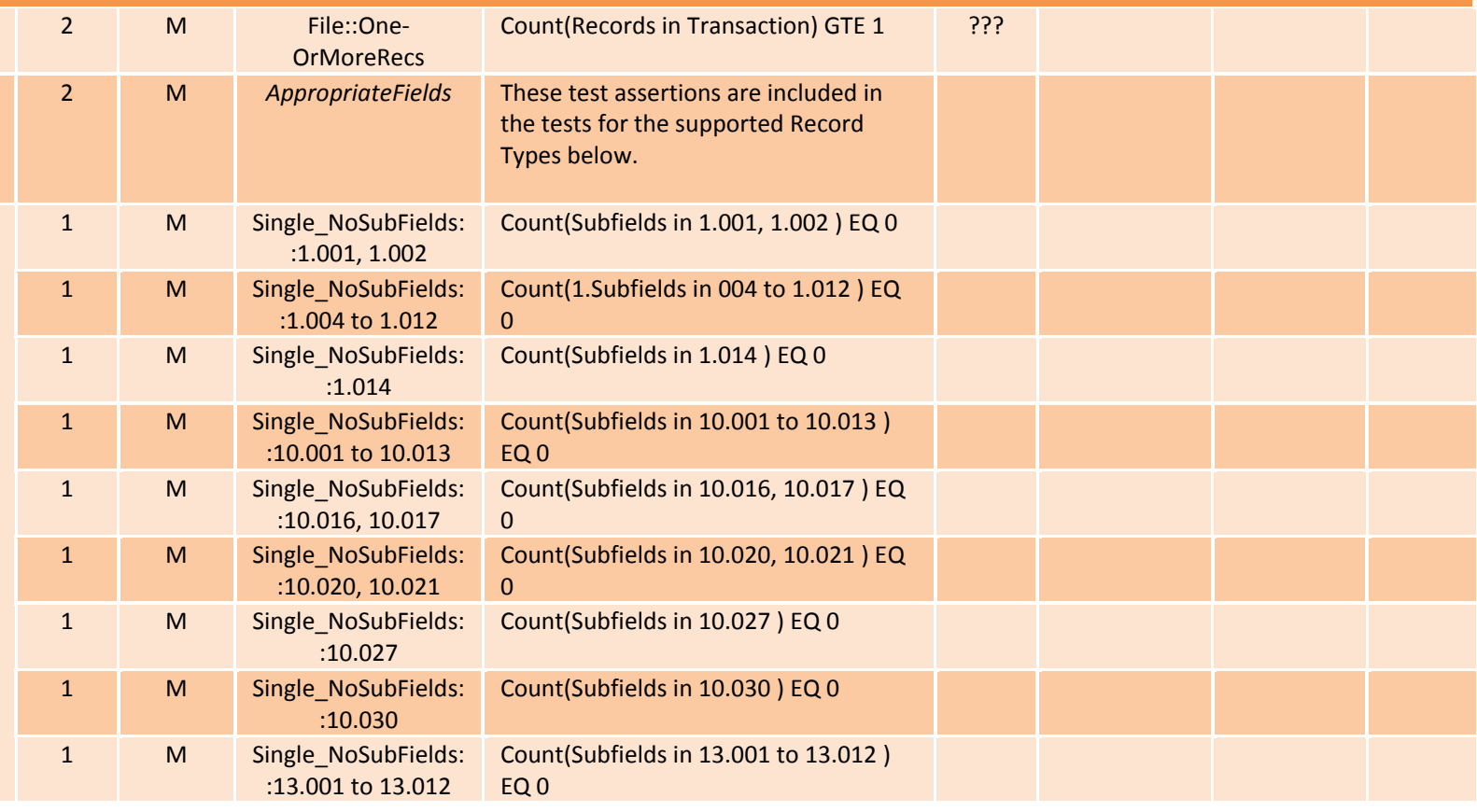


17.001 to $17.015,17.017,17.018$, 17.020 to $17.023,17.025,17.026$, 17.030

For tagged-field logical records each field shall begin with the logical record type number chosen from Table 4.

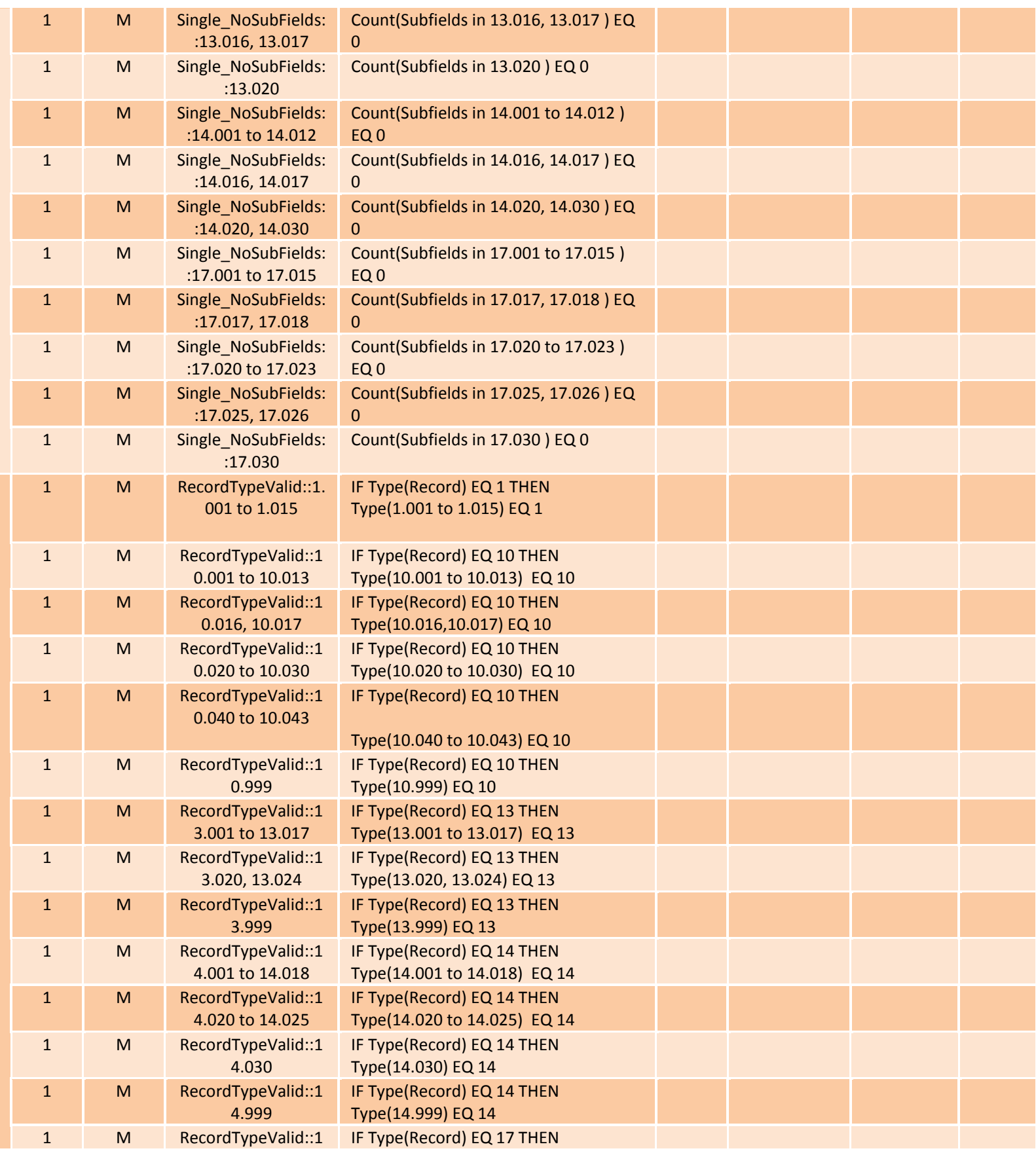




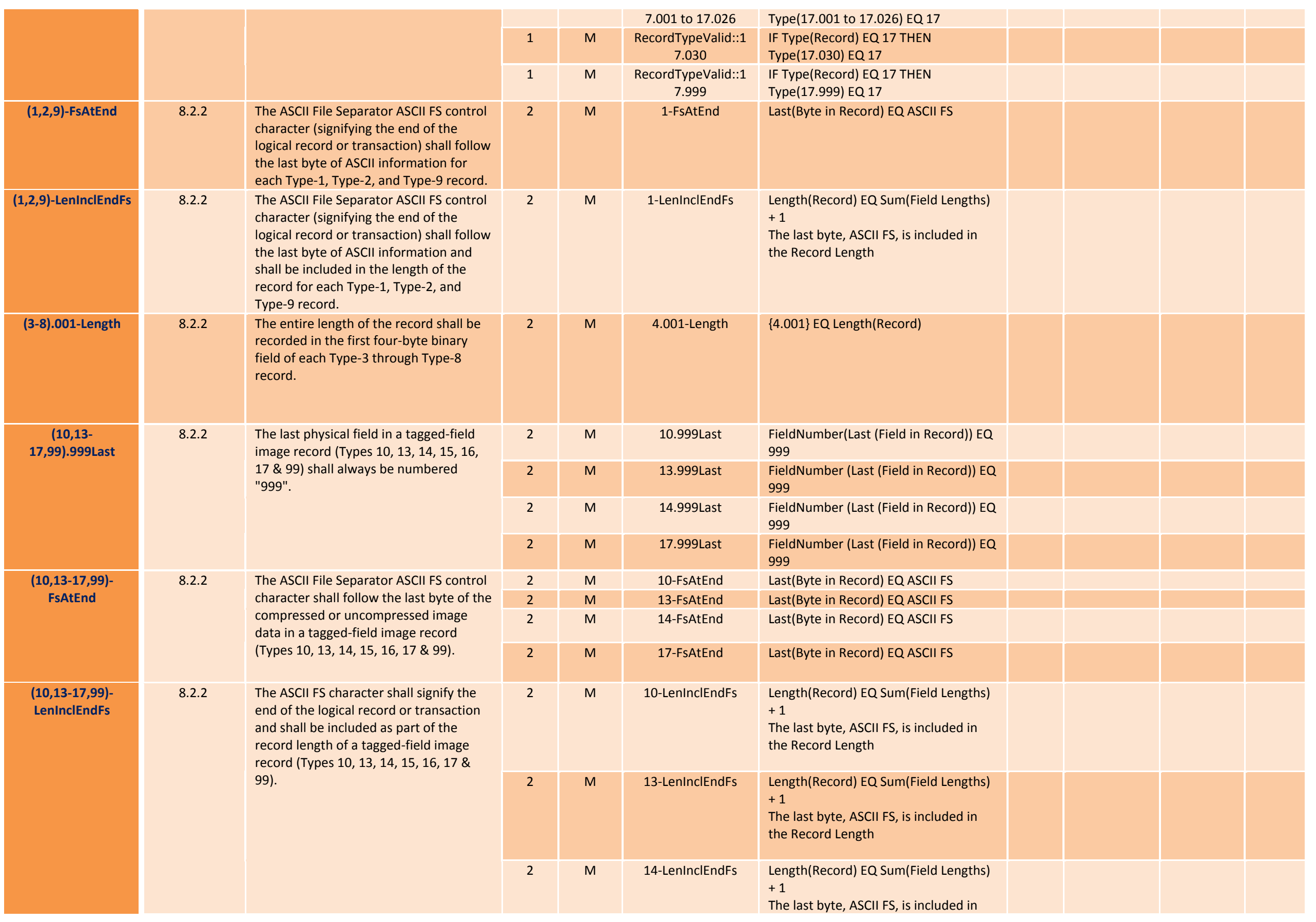




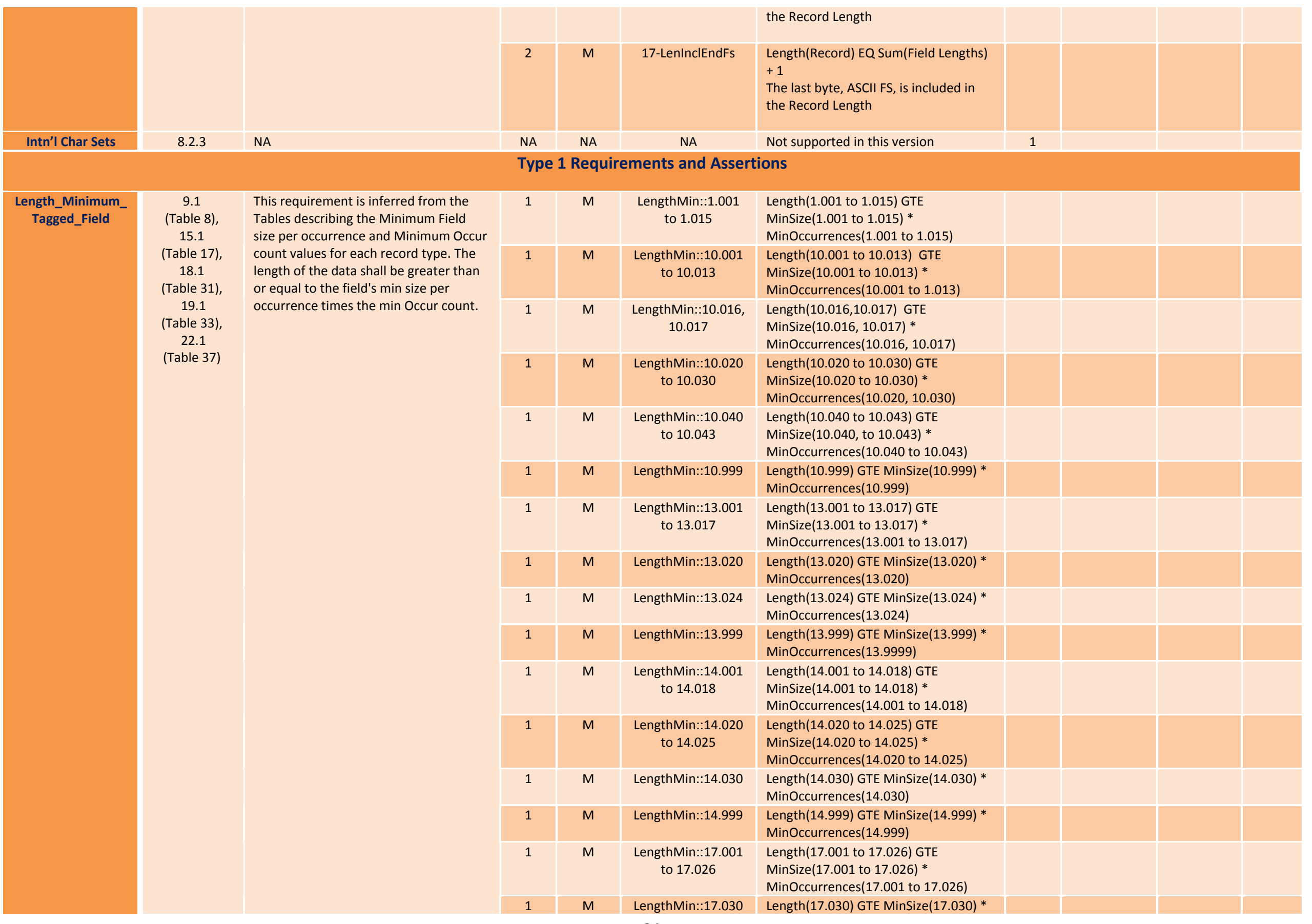



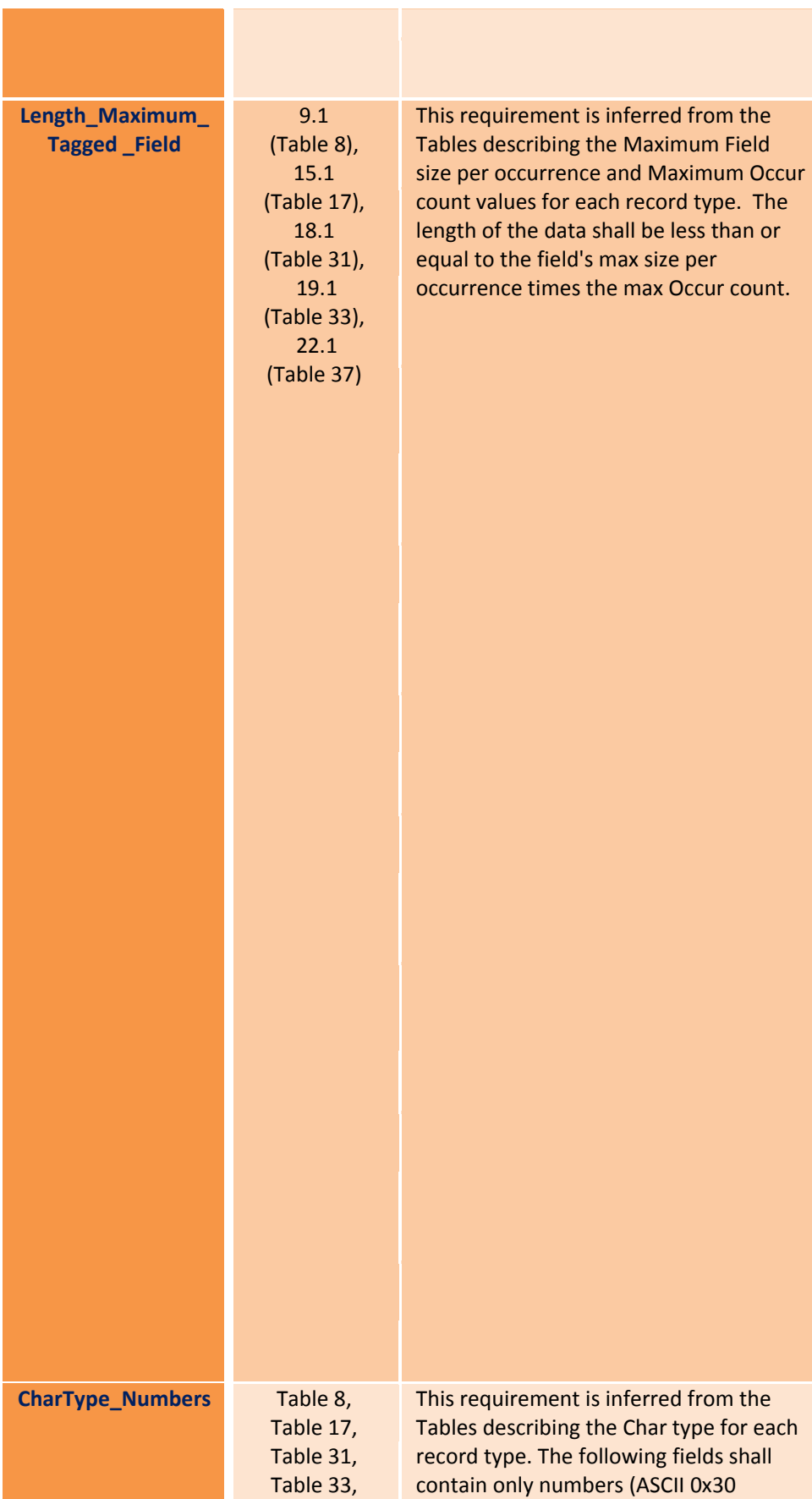

\begin{tabular}{|c|c|c|}
\hline 1 & M & LengthMin::17.999 \\
\hline 1 & M & $\begin{array}{l}\text { LengthMax::1.001 } \\
\text { to } 1.015\end{array}$ \\
\hline 1 & M & $\begin{array}{c}\text { LengthMax::10.001 } \\
\text { to } 10.013\end{array}$ \\
\hline 1 & M & $\begin{array}{c}\text { LengthMax::10.016, } \\
10.017\end{array}$ \\
\hline 1 & M & $\begin{array}{c}\text { LengthMax::10.020 } \\
\text { to } 10.030\end{array}$ \\
\hline 1 & M & $\begin{array}{c}\text { LengthMax::10.040 } \\
\text { TO } 10.043\end{array}$ \\
\hline 1 & M & LengthMax::10.999 \\
\hline 1 & M & $\begin{array}{c}\text { LengthMax::13.001 } \\
\text { TO } 13.017\end{array}$ \\
\hline 1 & M & LengthMax::13.020 \\
\hline 1 & M & LengthMax::13.024 \\
\hline 1 & M & LengthMax::13.999 \\
\hline 1 & M & $\begin{array}{c}\text { LengthMax::14.001 } \\
\text { TO } 14.018\end{array}$ \\
\hline 1 & M & $\begin{array}{c}\text { LengthMax::14.020 } \\
\text { TO } 14.025\end{array}$ \\
\hline 1 & M & LengthMax::14.030 \\
\hline 1 & M & LengthMax::14.999 \\
\hline 1 & M & $\begin{array}{c}\text { LengthMax::17.001 } \\
\text { TO } 17.026\end{array}$ \\
\hline 1 & M & LengthMax::17.030 \\
\hline 1 & M & LengthMax::17.999 \\
\hline 1 & M & $\begin{array}{c}\text { CharType_Numbers } \\
:: 1.001,1.002\end{array}$ \\
\hline 1 & M & $\begin{array}{c}\text { CharType_Numbers } \\
:: 1.005,1.006\end{array}$ \\
\hline
\end{tabular}

MinOccurrences(17.030)

Length(17.999) GTE MinSize(17.999) *

MinOccurrences(17.999)

Length(1.001 to 1.015) LTE

MaxSize(1.001 to 1.015$)$ *

MaxOccurrences(1.001 to 1.015 )

Length(10.001 to 10.013) LTE

MaxSize(10.001 to 10.013) *

MaxOccurrences(10.001 to 1.013)

MaxSize $(10.016,10.017)$ *

MaxOccurrences $(10.016,10.017)$

Length(10.020 to 10.030) LTE

MaxSize(10.020 to 10.030) *

MaxOccurrences $(10.020,10.030)$

ength(10.040 to 10.043) LTE

MaxSize(10.040, to 10.043) *

MaxOccurrences(10.040 to 10.043)

ength(10.999) LTE MaxSize(10.999) *

MaxOccurrences(10.999)

Length(13.001 to 13.017) LTE

MaxSize(13.001 to 13.017) *

MaxOccurrences(13.001 to 13.017)

Length(13.020) LTE MaxSize(13.020) *

MaxOccurrences(13.020)

ength(13.024) LTE MaxSize(13.024) *

MaxOccurrences(13.024)

Length(13.999) LTE MaxSize(13.999) *

MaxOccurrences(13.9999)

ength(14.001 to 14.018) LTE

MaxSize(14.001 to 14.018 ) *

MaxOccurrences(14.001 to 14.018)

Length(14.020 to 14.025) LTE

axSize(14.020 to 14.025$)$ *

MaxOccurrences(14.020 to 14.025 )

Length(14.030) LTE MaxSize(14.030) *

MaxOccurrences(14.030)

ength(14.999) LTE MaxSize(14.999) *

MaxOccurrences(14.999)

Length(17.001 to 17.026) LTE

MaxSize(17.001 to 17.026) *

MaxOccurrences(17.001 to 17.026 )

ength(17.030) LTE MaxSize(17.030) *

MaxOccurrences(17.030)

MaxOccurrences(17.999)

record type. The following fields shal

Table 33,

contain only numbers (ASCII 0x30 


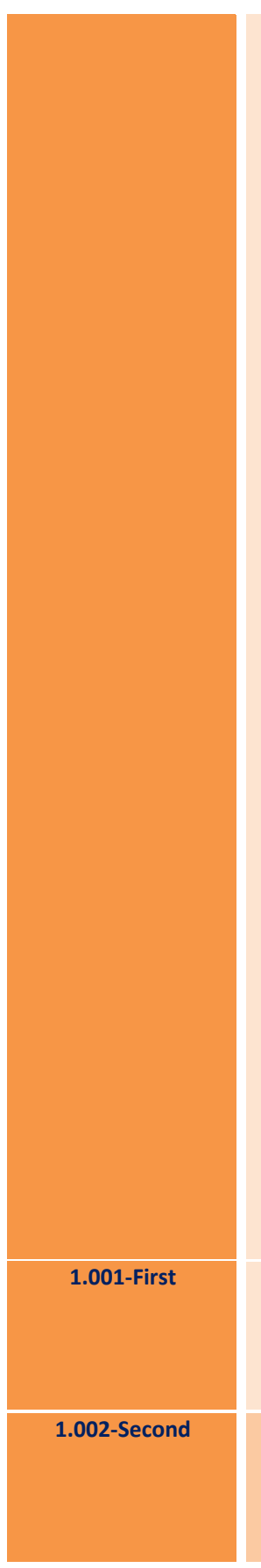

through 0x39):

$1.001,1.002,1.005,1.006$

$10.001,10.002,10.005$ to

$10.009,10.013,10.016,10.017$

13.001 to $13.003,13.005$ to 13.010 ,

$13.012,13.013,13.016,13.017$

14.001 to $14.003,14.005$ to 14.010 ,

$14.012,14.016,14.017$

17.001 to $17.003,17.005$ to 17.010 ,

$17.012,17.020,17.022,17.023,17.025$

17.026

Within a Type-1 logical record, entries shall be provided in numbered fields. It is required that the first two fields of the record are ordered.1.001 must be the first field.

9.1 Within a Type-1 logical record, entries Within a Type- 1 logical record, entries
shall be provided in numbered fields. It is required that the first two fields of the record are ordered. 1.002 must be the second field.

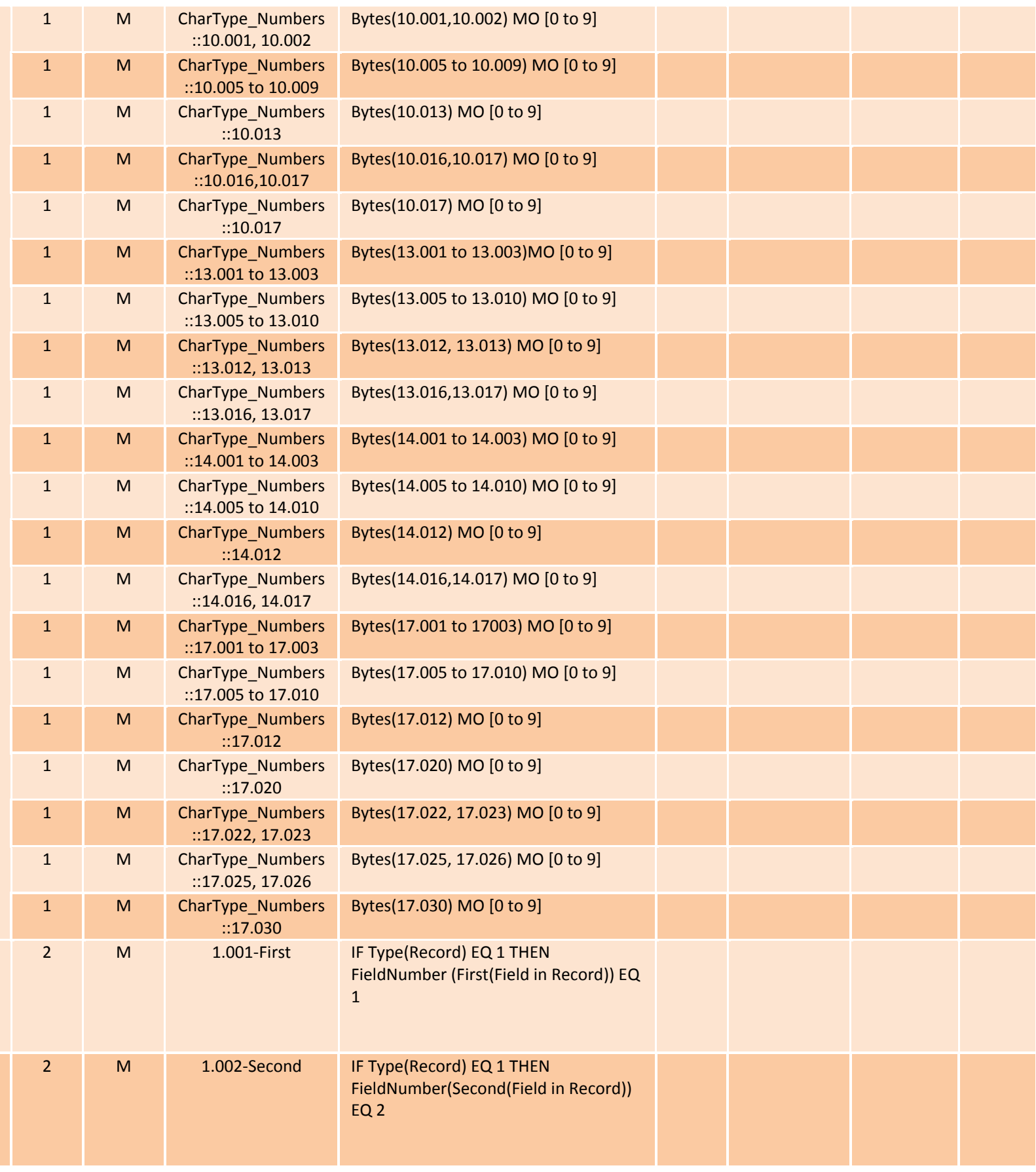




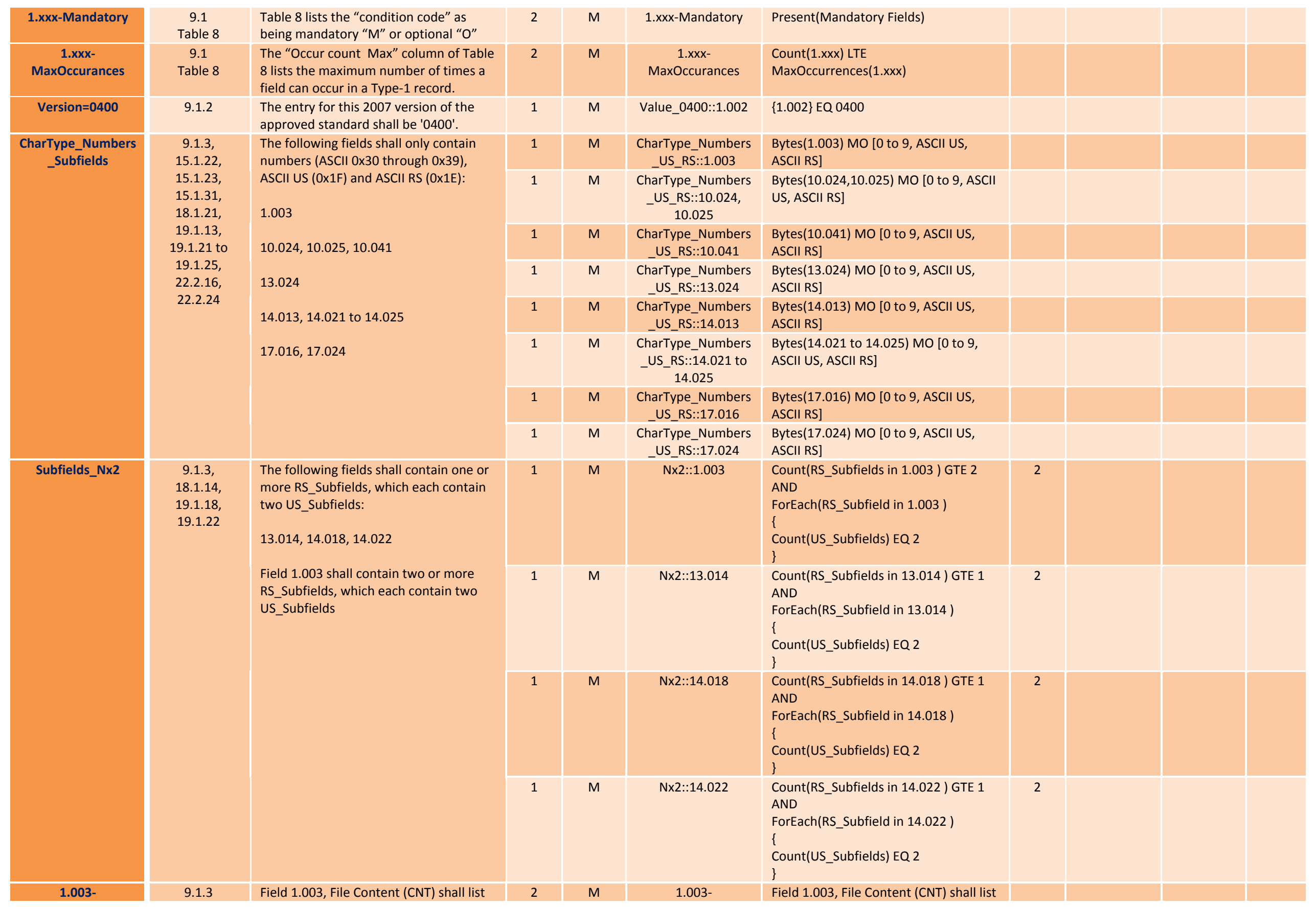




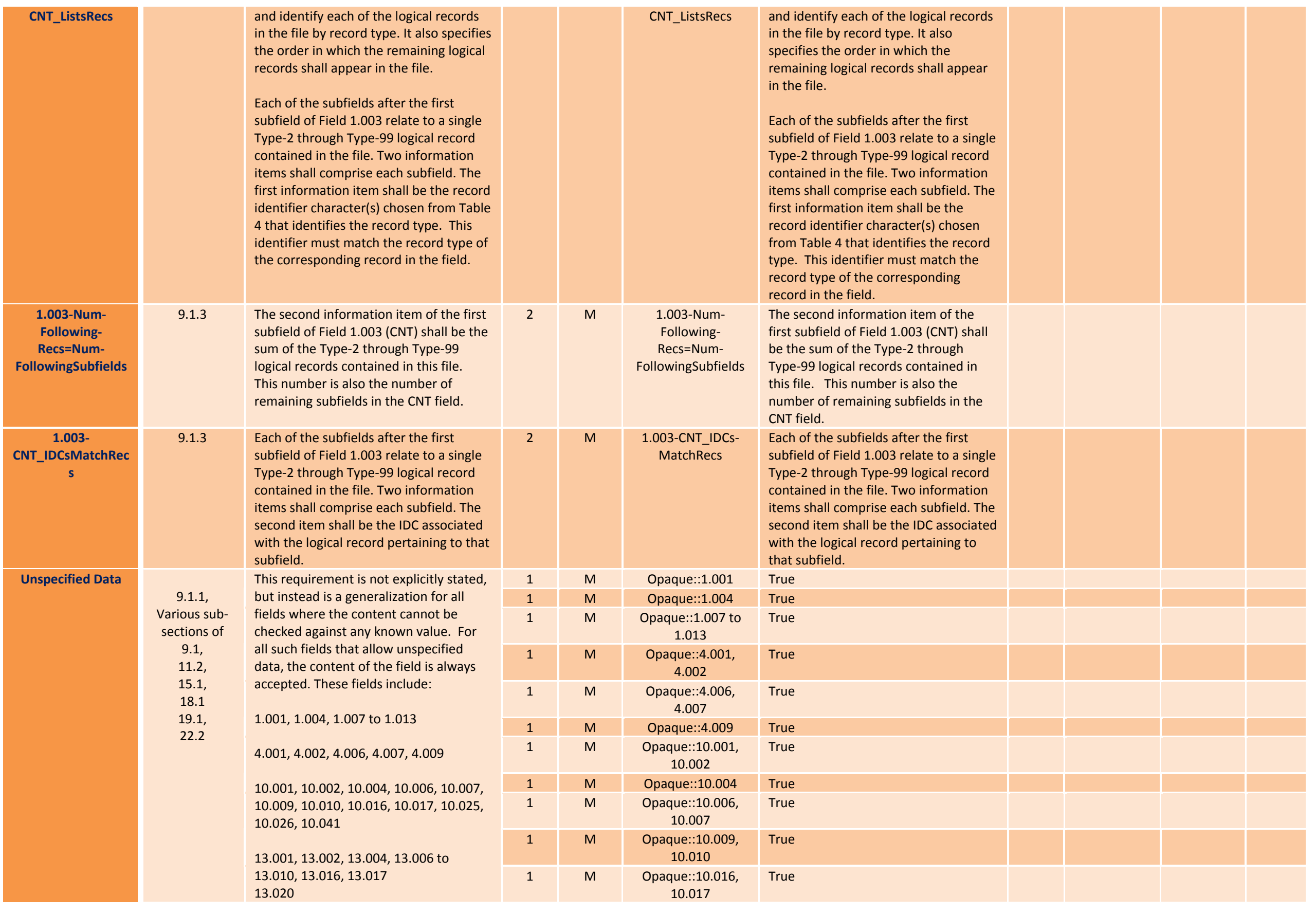




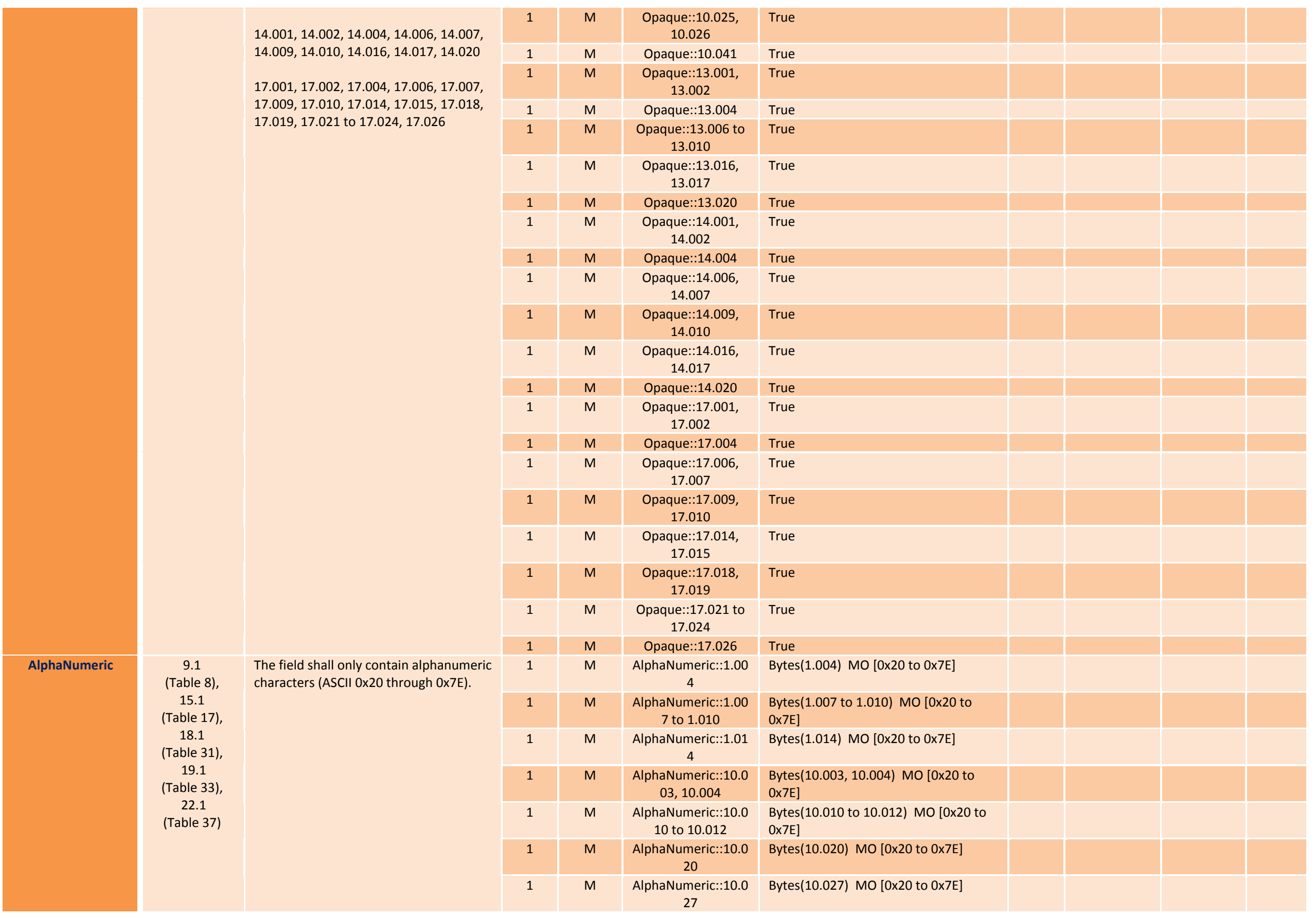




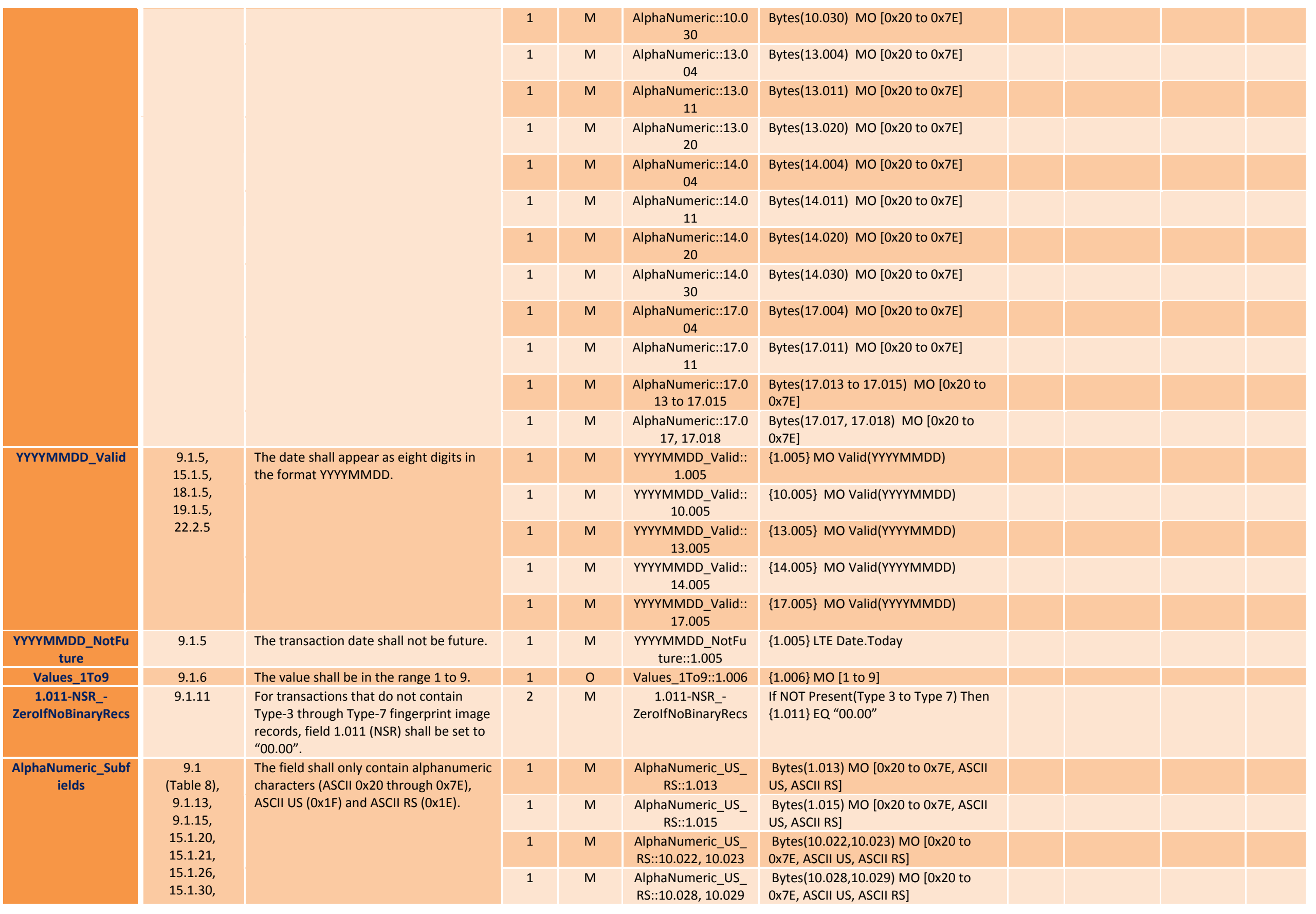




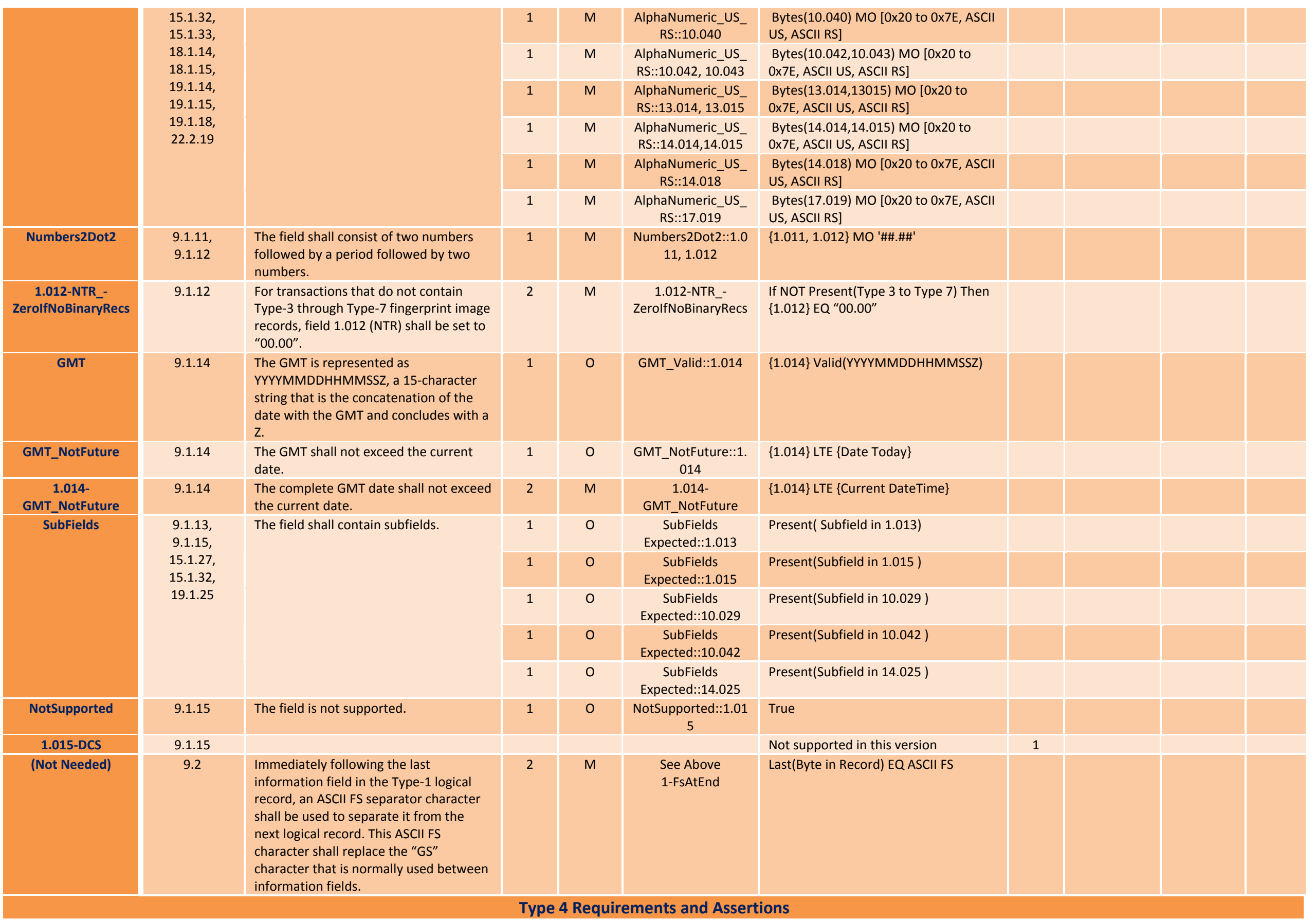









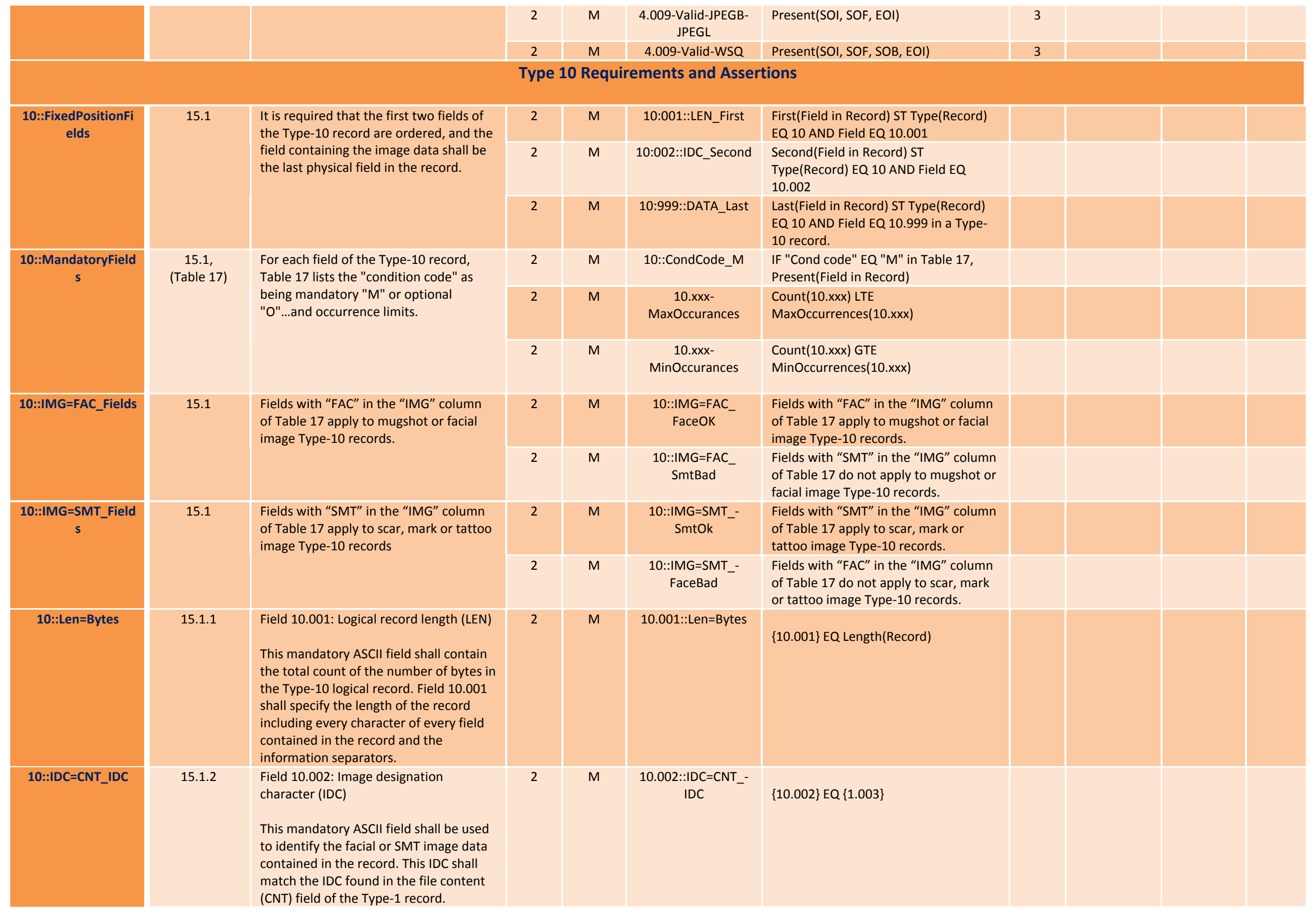




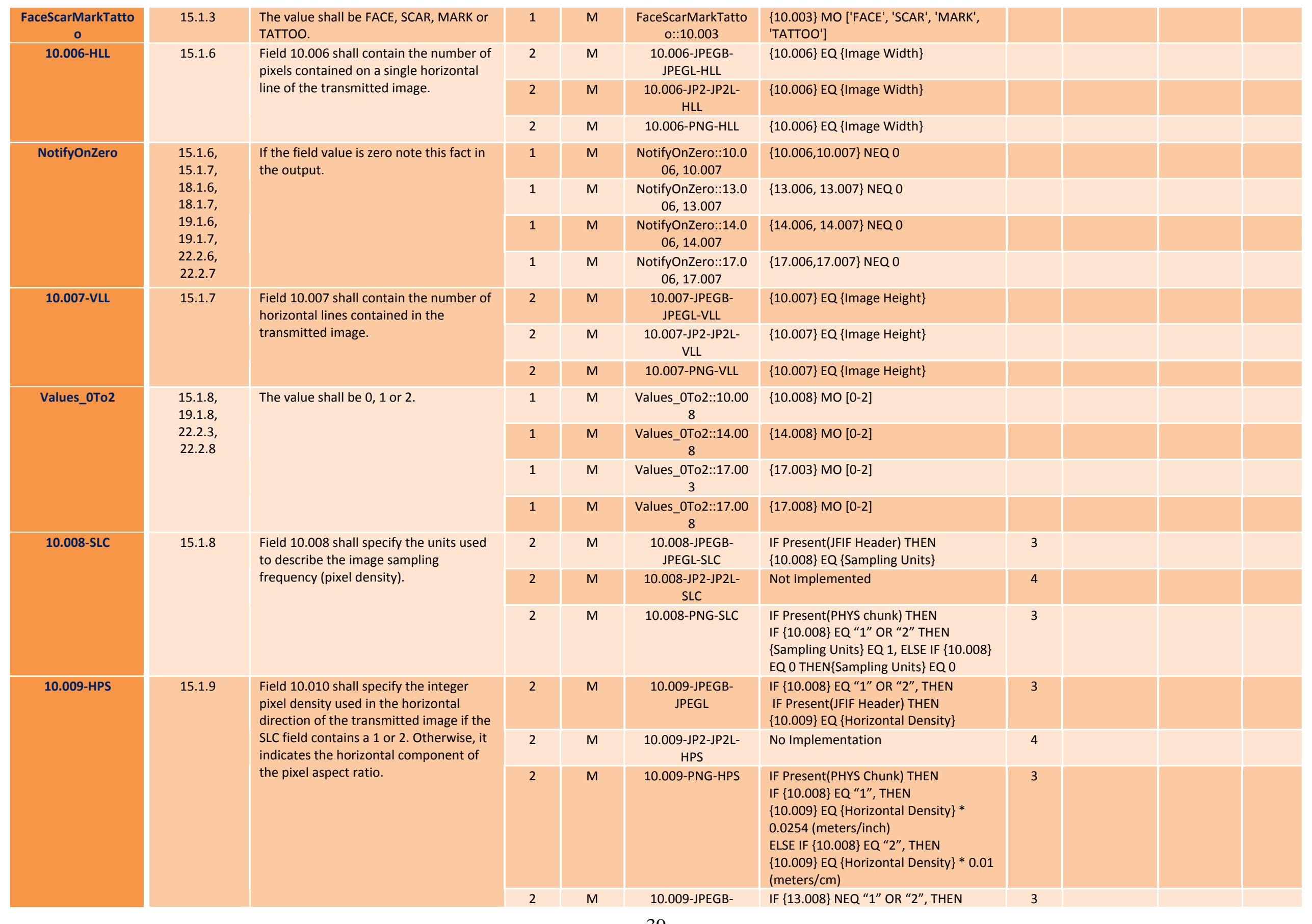




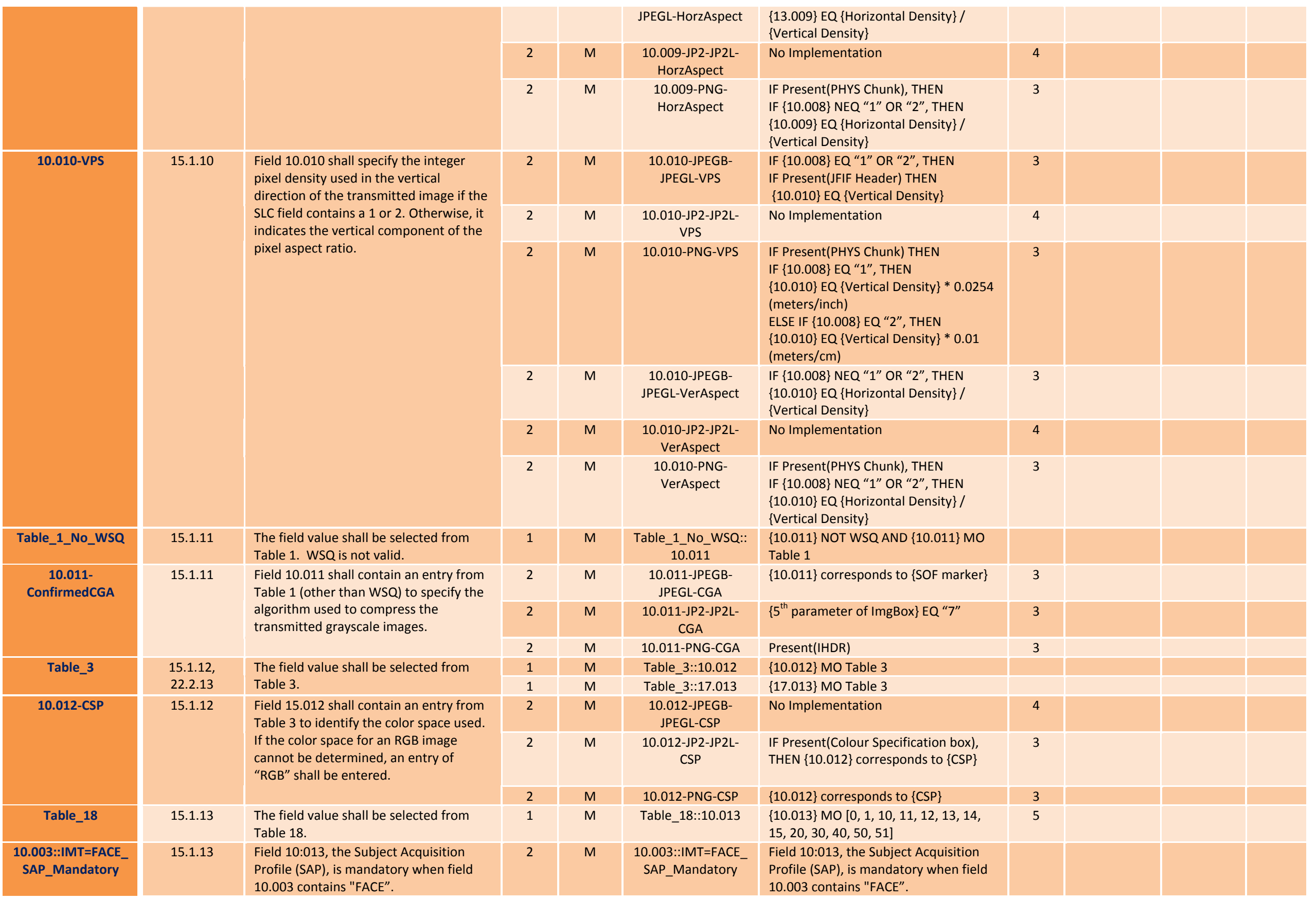




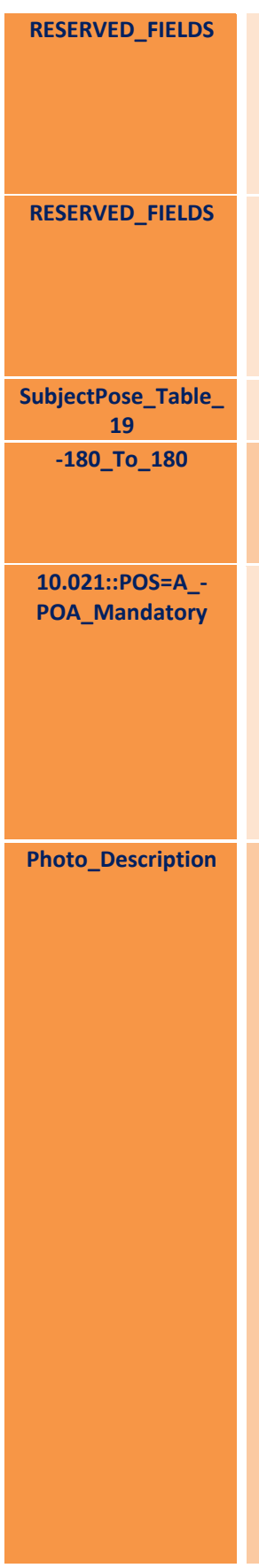

15.1.14

Fields 10.014 and 10.015 are reserved for definition and inclusion in future

revisions of this standard. None of these

fields are to be used at this revision

level. If any of these fields are present,

they are to be ignored.

15.1.17 Fields 10.018 and 10.019 are reserved

for definition and inclusion in future

revisions of this standard. None of these

fields are to be used at this revision

level. If any of these fields are present,

they are to be ignored.

15.1.18 The value shall be selected from Table 19.

\begin{tabular}{l|l} 
15.1.19 & The offset angle shall be measured from
\end{tabular} the full-face pose position and have a range of values from -180 degrees to +180 degrees.

15.1.19 Field 10.021, Pose offset angle (POA), shall only be used for the exchange of facial image data if Field 10.020 (POS) contains an "A" to indicate an angled pose of the subject. This field should be omitted for a full face or a profile. If the entry in the POS field is an "F", "L", or

"R", the contents of this field are ignored.

\begin{tabular}{l|l} 
15.1.20 & This optional ASCII field, retained for
\end{tabular} legacy systems, is used for the exchang of facial image data. When present, it shall consist of one or more subfields and shall describe special attributes of the captured facial image. Attributes associated with the facial image may be selected from Table 20 and entered in this field as one or more subfields separated by the ASCII RS separator character between the items. Physical characteristics, such as FRECKLES may be entered as a subfield consisting of two information items. The first is PHYSICAL followed by the ASCII US separator,

followed by the characteristic as listed in the Ninth (or current) Edition of the NCIC Code Manual, December, 2000. The OTHER category is used to enter unlisted or miscellaneous attributes of the facial image. This information shall be entered as a two-information item subfield. The first is OTHER followed by the ASCII US separator, followed by the
10.014::Reserved

\begin{tabular}{|c|c|c|}
\hline- & - & $10.015::$ Reserved \\
\hline- & - & $10.018::$ Reserved \\
\hline- & - & $10.019::$ Reserved \\
\hline & & \\
\hline 1 & 0 & $\begin{array}{c}\text { SubjectPose::10.02 } \\
0\end{array}$ \\
\hline
\end{tabular}

\begin{tabular}{|c|c|c|}
\hline 1 & 0 & $\begin{array}{c}- \\
180 \_T o \_180:: 10.02 \\
1\end{array}$ \\
\hline 2 & 0 & $\begin{array}{l}\text { 10.021::POS=A_- } \\
\text { POA_Mandatory }\end{array}$ \\
\hline
\end{tabular}
180

\section{IF $\{10.020\} E Q$ "A" THEN}

Present(10.021)

Present(10.015)

Present(10.018)

Present(10.019)

$\{10.020\} \mathrm{MO}$ ['F', 'R', 'L', 'A', 'D']

10.021\} LTE 180 AND $\{10.021\}$ GTE -

5

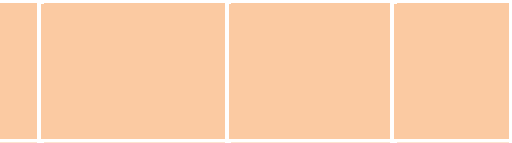

POA Mandatory

\begin{tabular}{|c|c|c|}
\hline 1 & 0 & Photo_Descriptors:: \\
\hline 10.022 \\
\hline
\end{tabular}

$\{10.022\}$ MO Table 20 


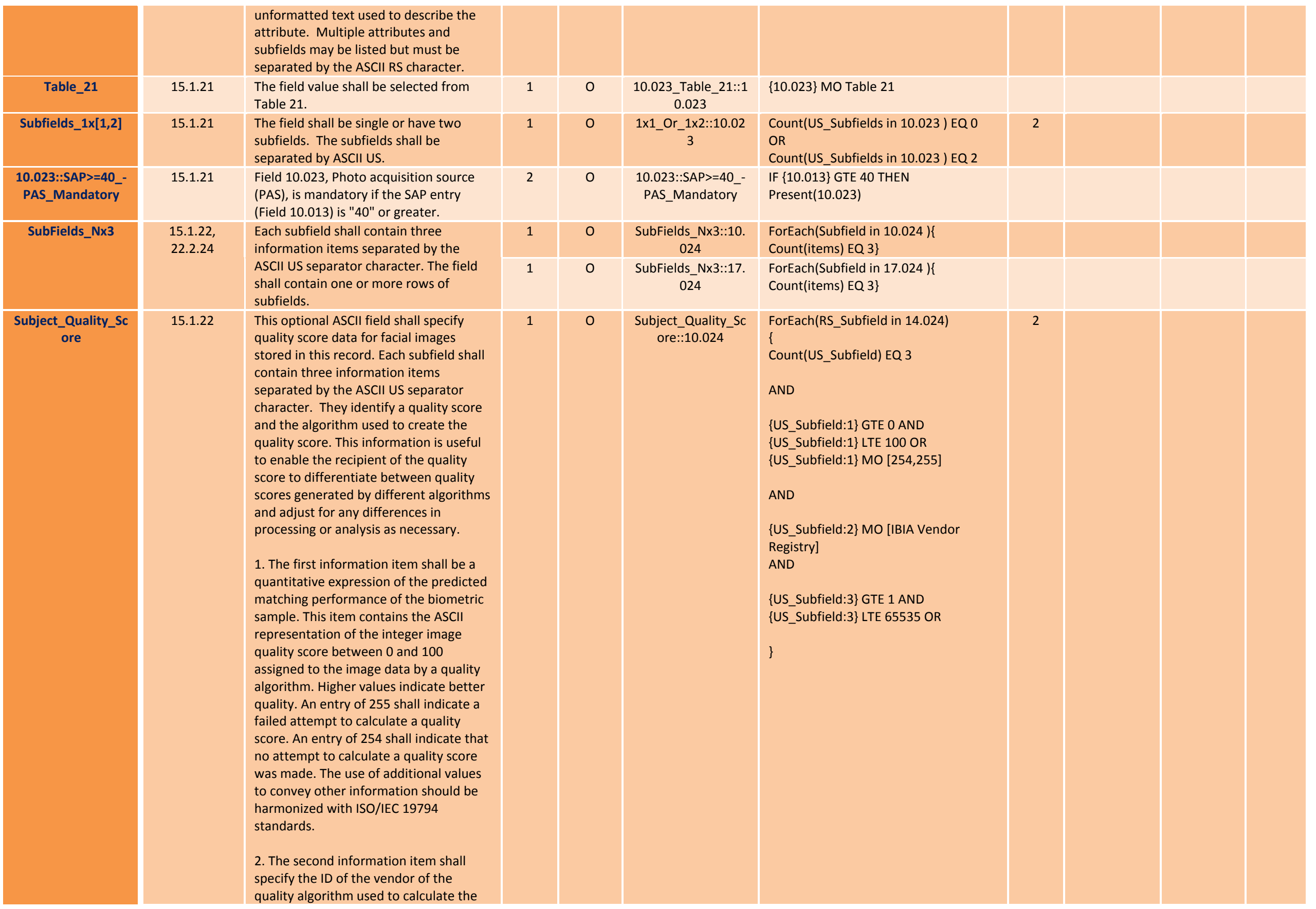




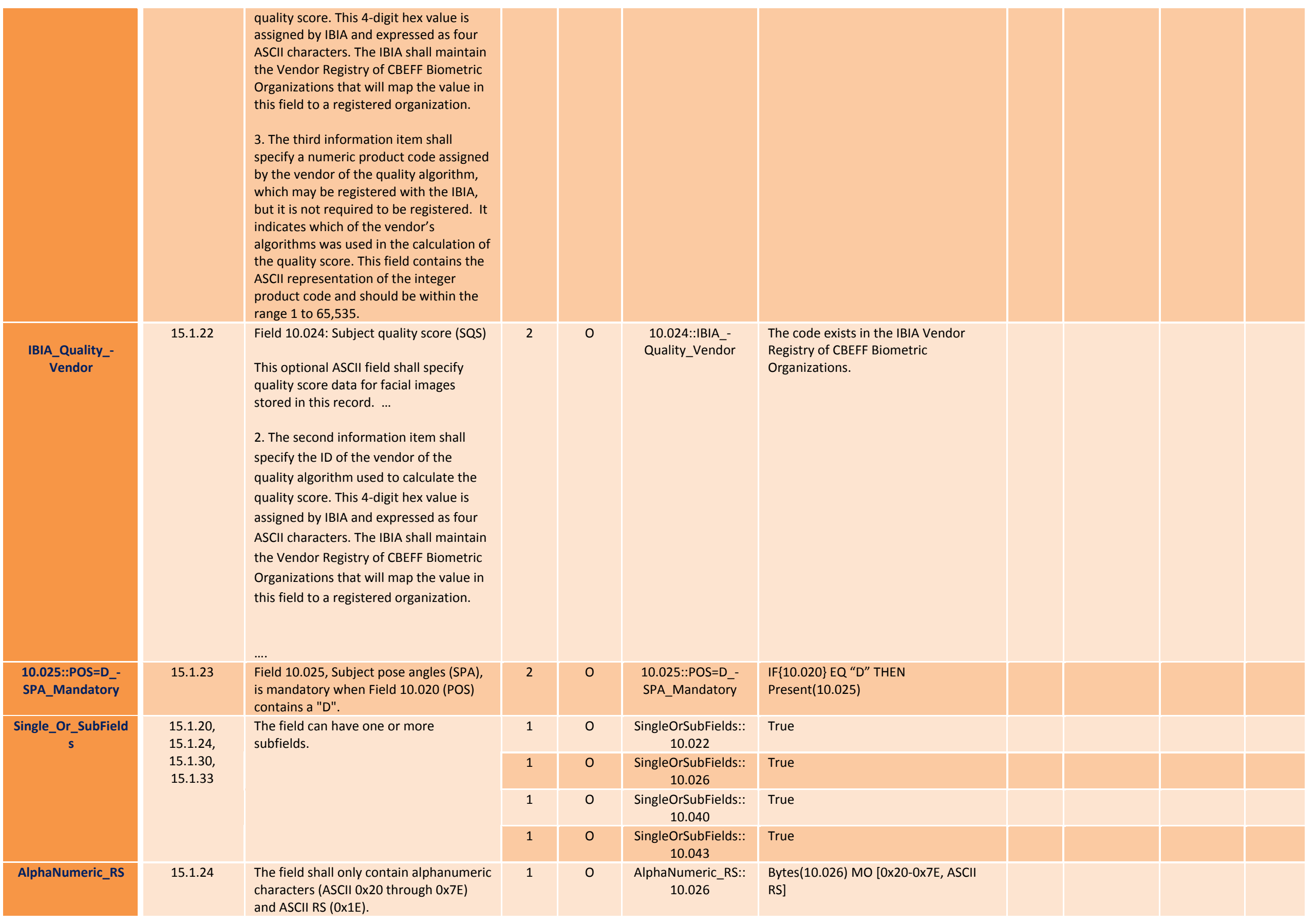




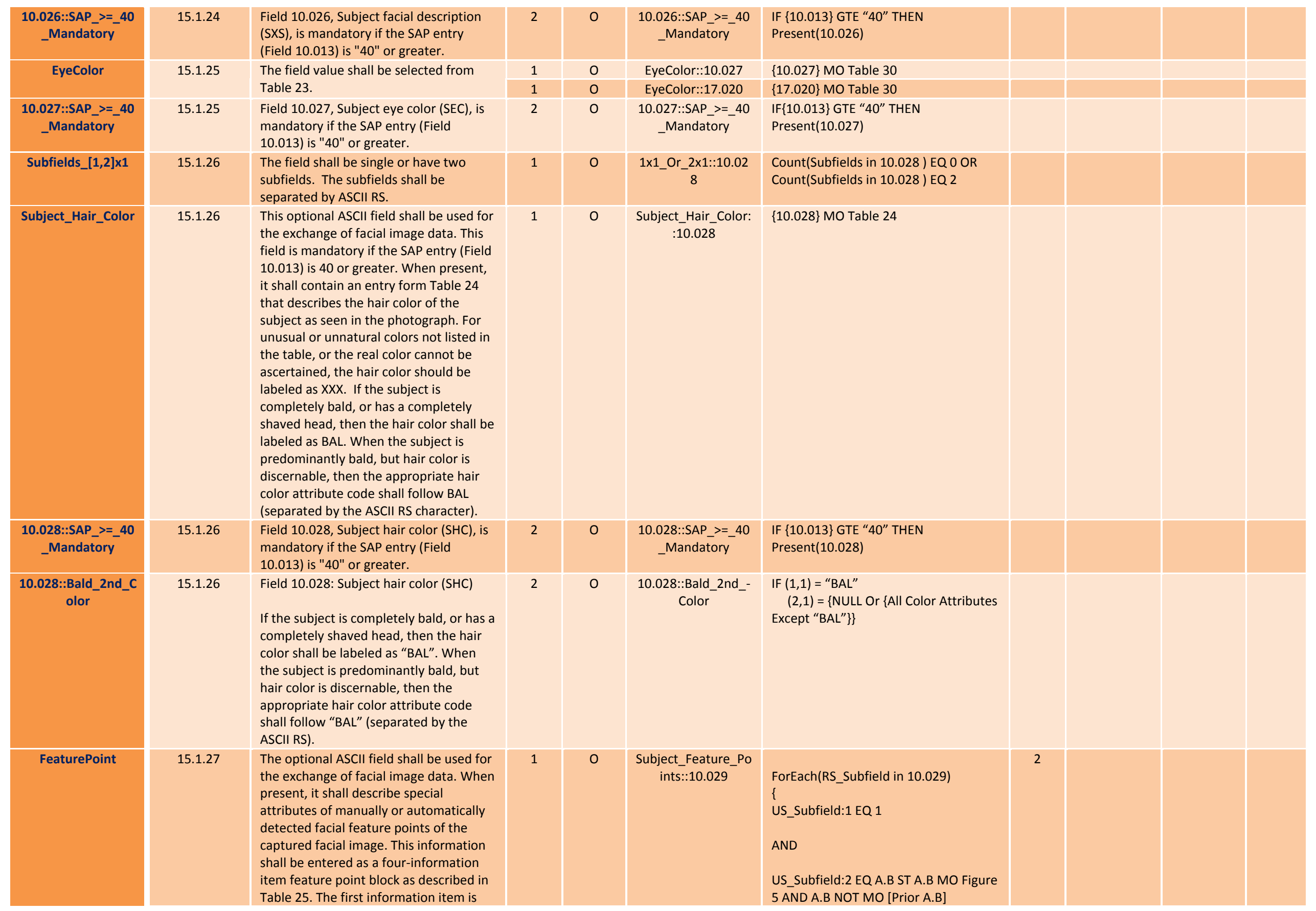




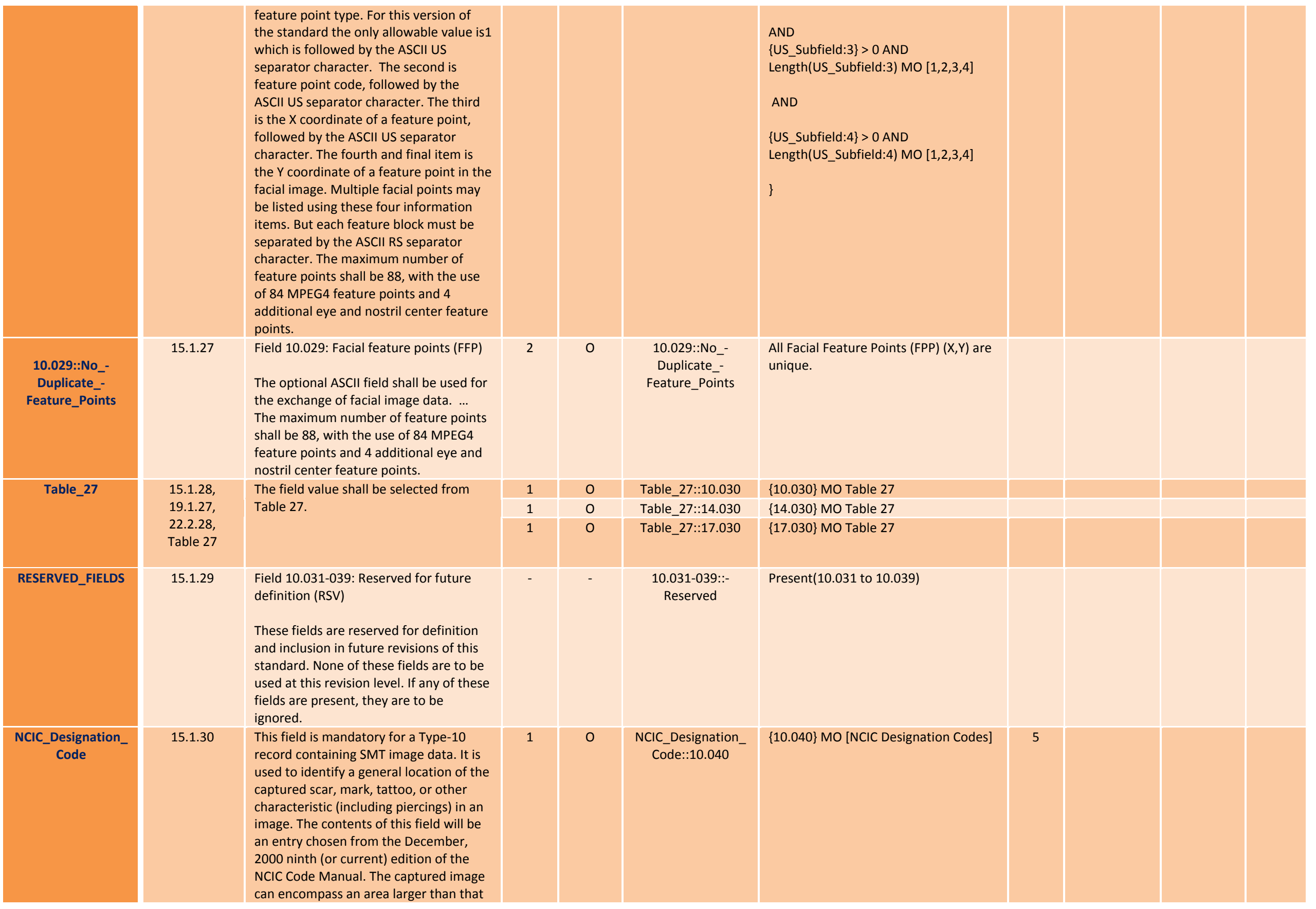




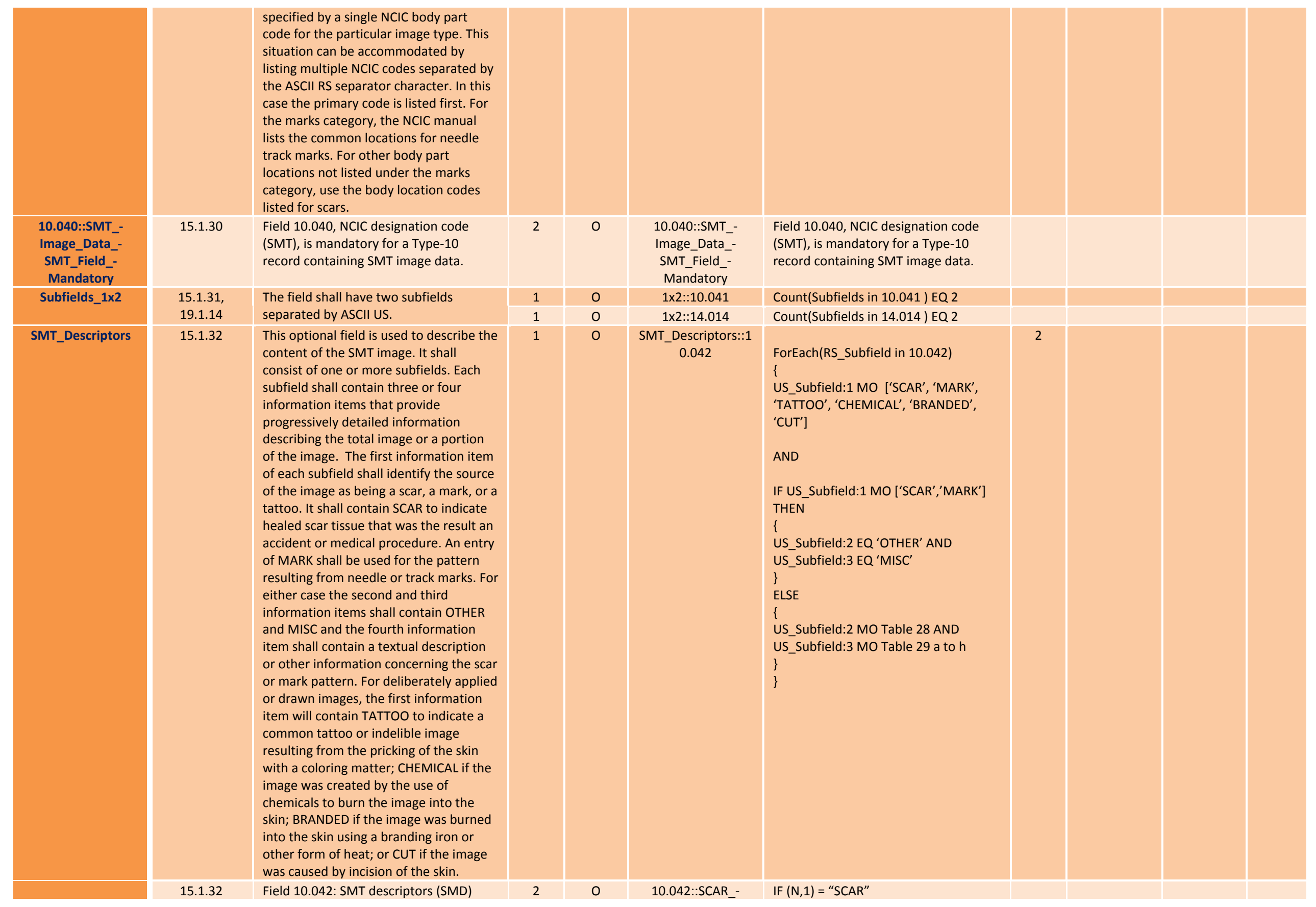




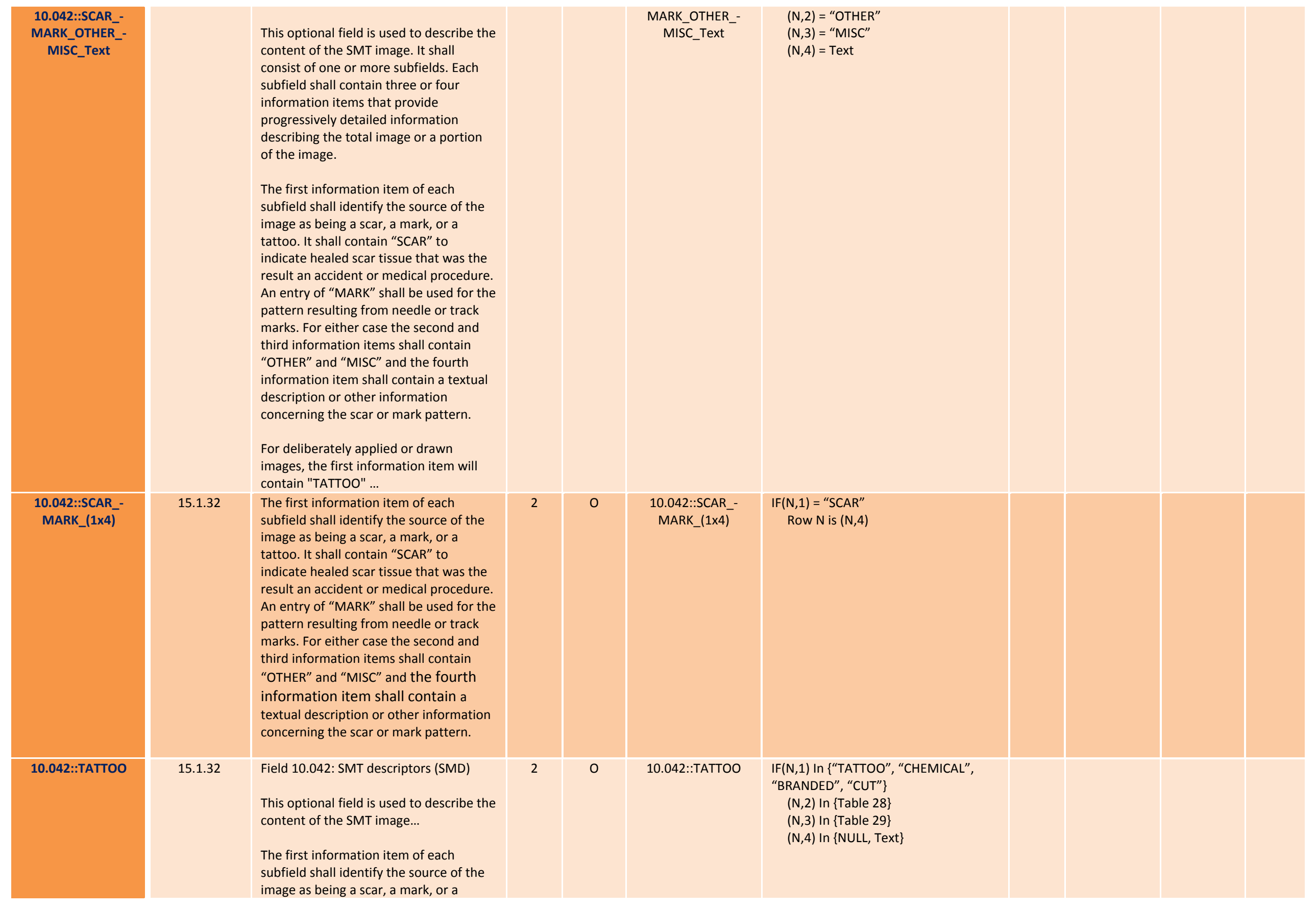




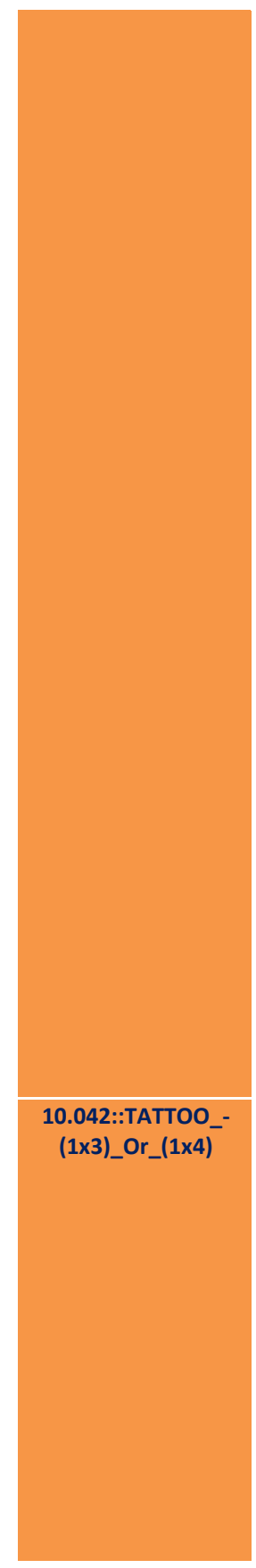

tattoo. It shall contain "SCAR" ...

For deliberately applied or drawn

images, the first information item will

contain "TATTOO" to indicate a common

tattoo or indelible image resulting from

the pricking of the skin with a coloring

matter; "CHEMICAL" if the image was

created by the use of chemicals to burn

the image into the skin; "BRANDED" if

the image was burned into the skin

using a branding iron or other form of

heat; or "CUT" if the image was caused

by incision of the skin.

The second information item shall be

the general class code of tattoo chosen

from Table 28. For each general class of

tattoo, there are several defined

subclasses. The third information item of

the subfield shall be the appropriate

subclass code selected from Table 29 a-h

which lists the various subclasses of

tattoos for each of the general classes.

The final and optional information item

in this subfield shall be an ASCll text

string that provides additional qualifiers

to describe the image or portion of the

image. For example, to fully describe a

tattoo, there may be a class description

of "ANIMAL", with a subclass description

of "DOG", and qualified by "golden

retriever with an overbite". The ASCII US

separator character will be used

between information items.

\begin{tabular}{l|l} 
15.1.32 Field 10.042: SMT descriptors (SMD) \\
\hline
\end{tabular}

This optional field is used to describe the content of the SMT image...

The first information item of each subfield shall identify the source of the image as being a scar, a mark, or a tattoo. It shall contain "SCAR" ...

For deliberately applied or drawn images, the first information item will contain "TATTOO" to indicate a ...

The final and optional information



IF (N,1) In \{"TATTOO" "CHEMICAL",

"BRANDED", "CUT"

$(\mathrm{N}, 2)$ Is Not NULL

$(\mathrm{N}, 3)$ Is Not NULL

$(N, 4)$ Is NULL Or Not NULL

(Optional) 


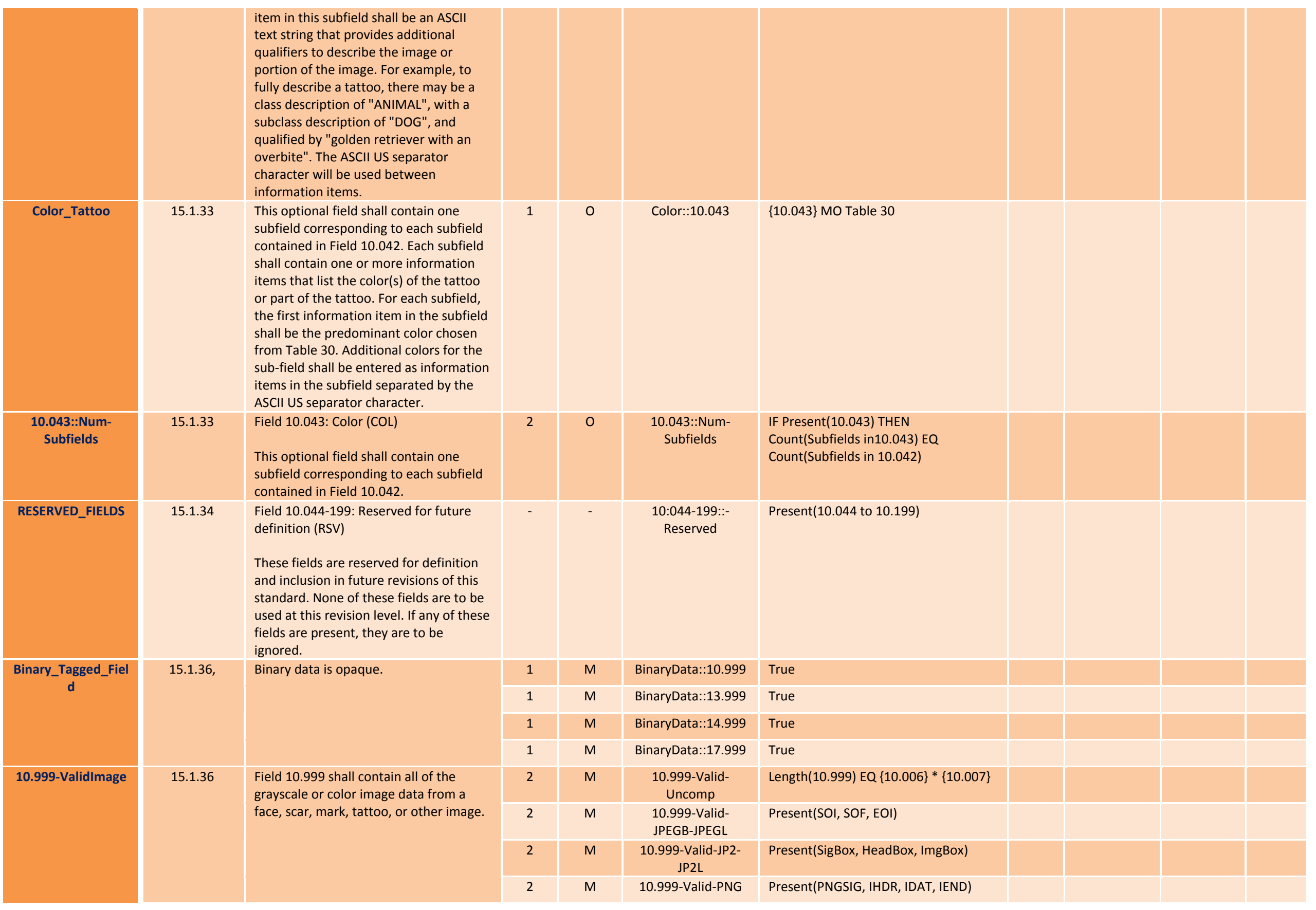




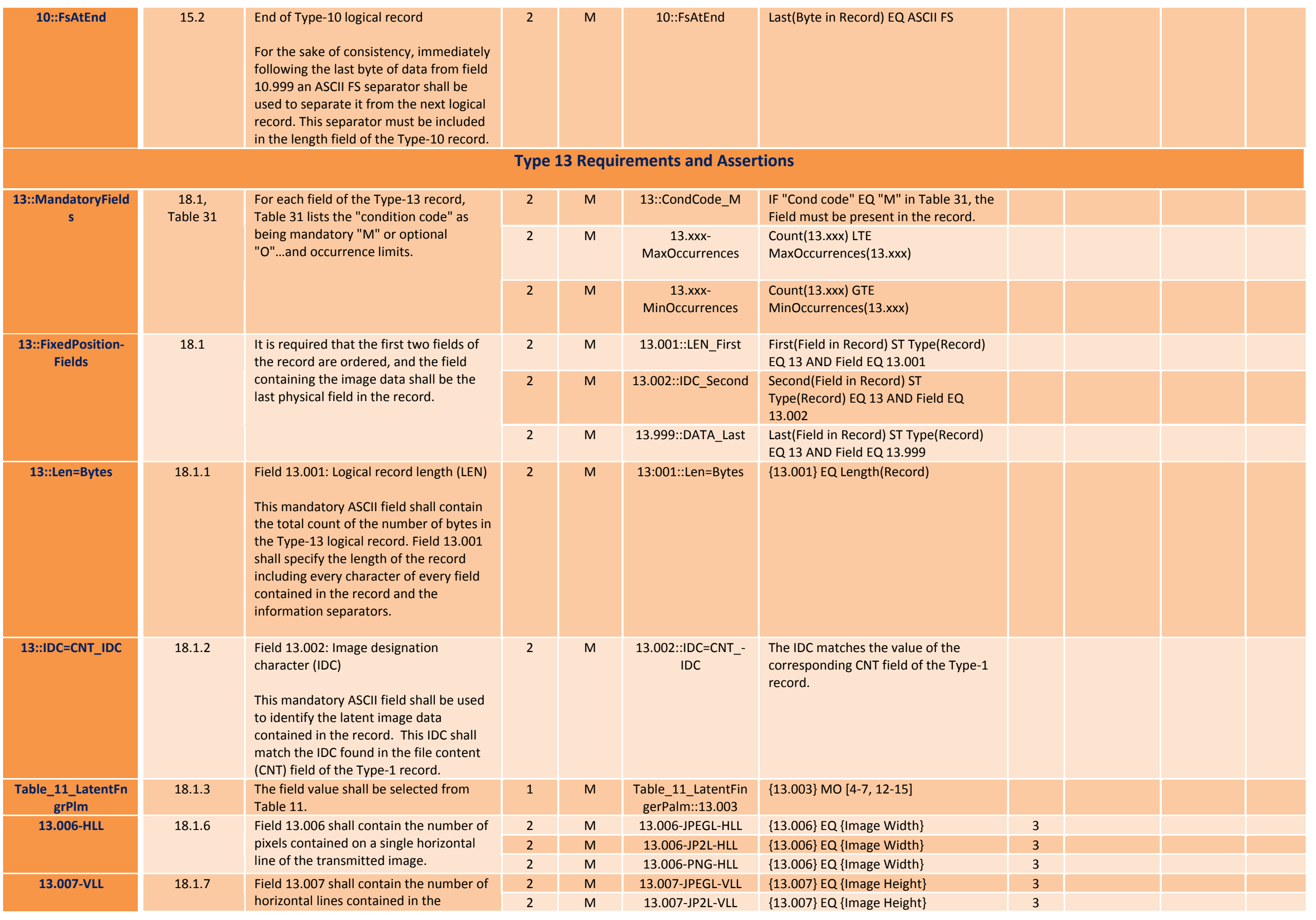




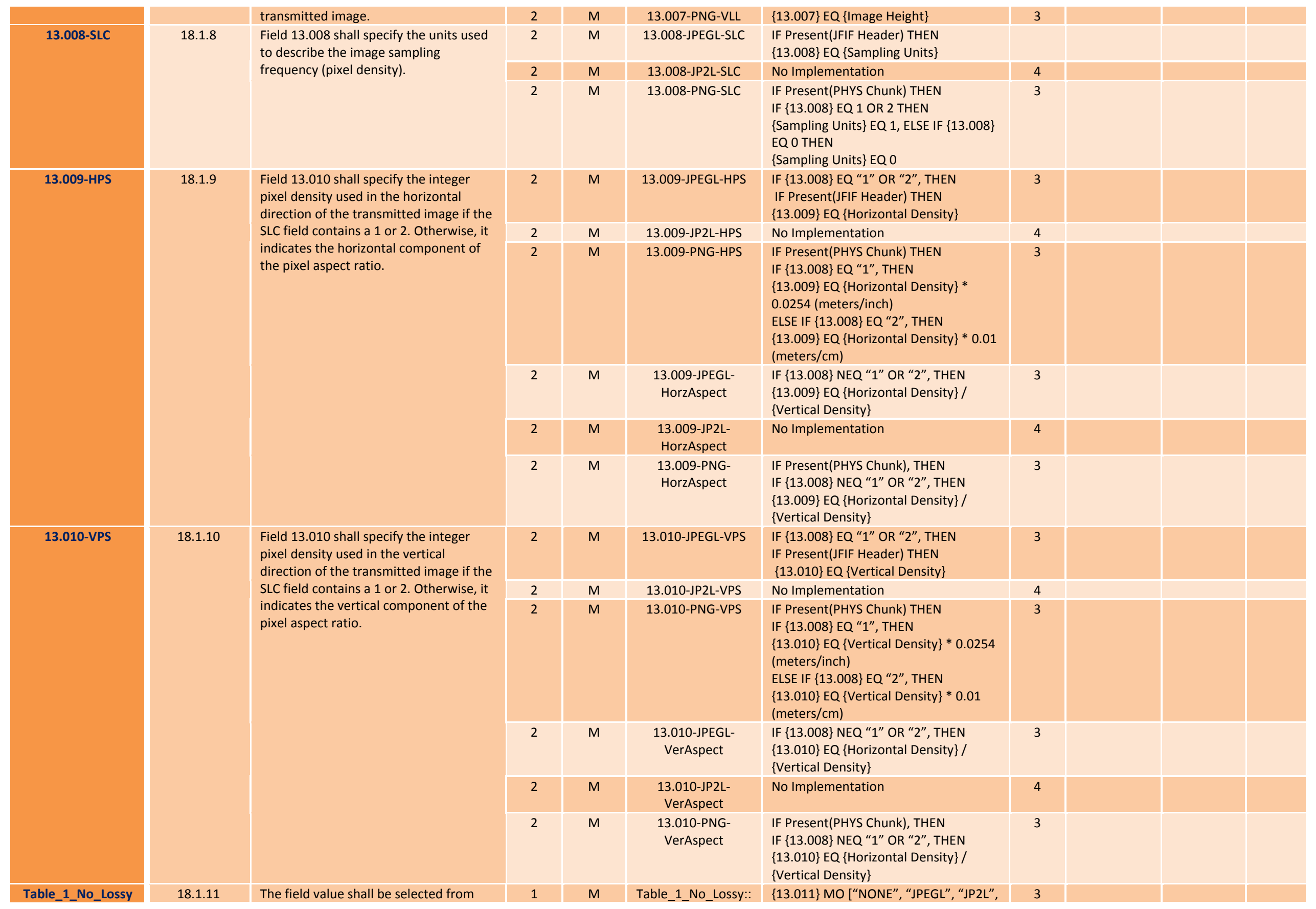




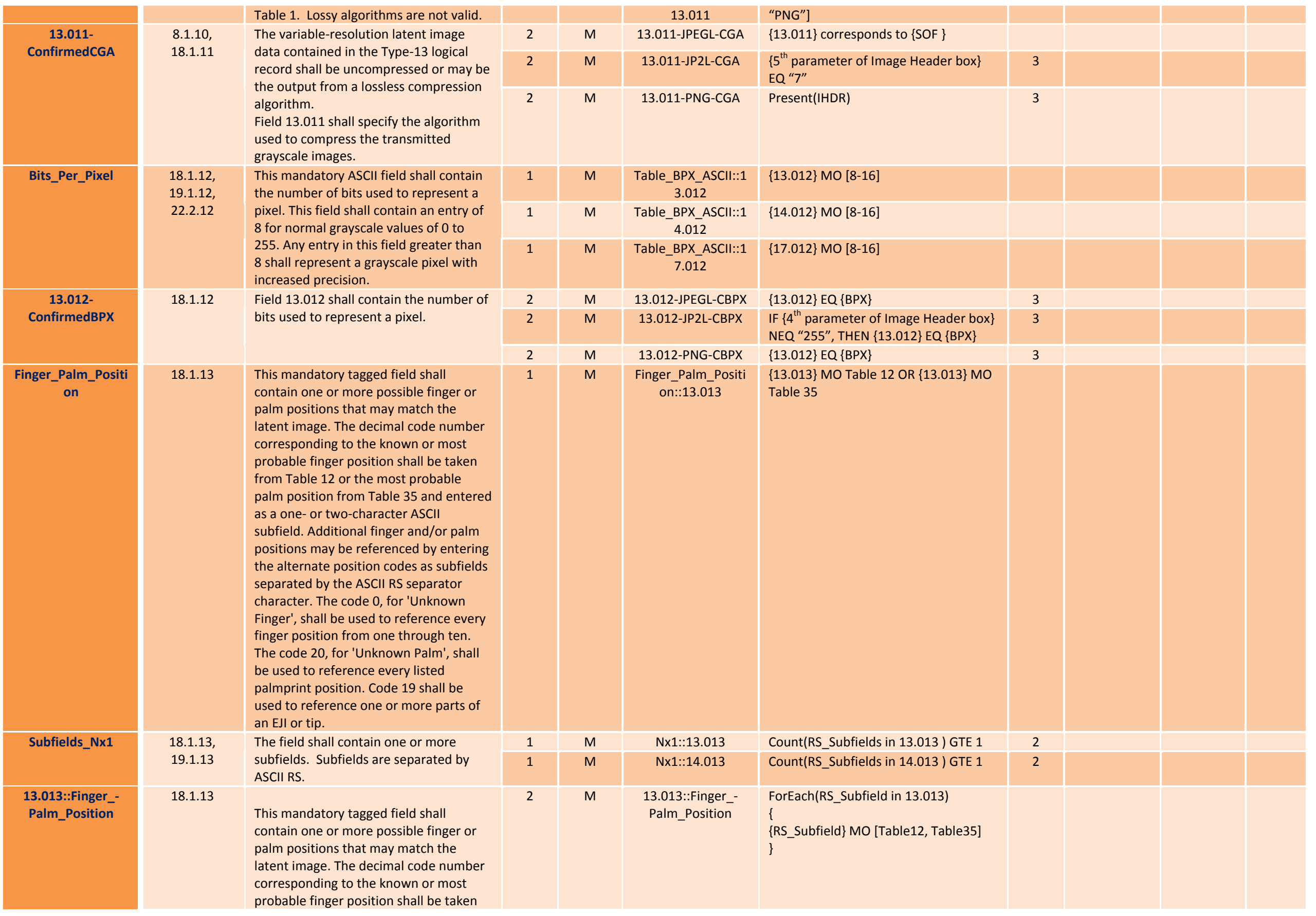




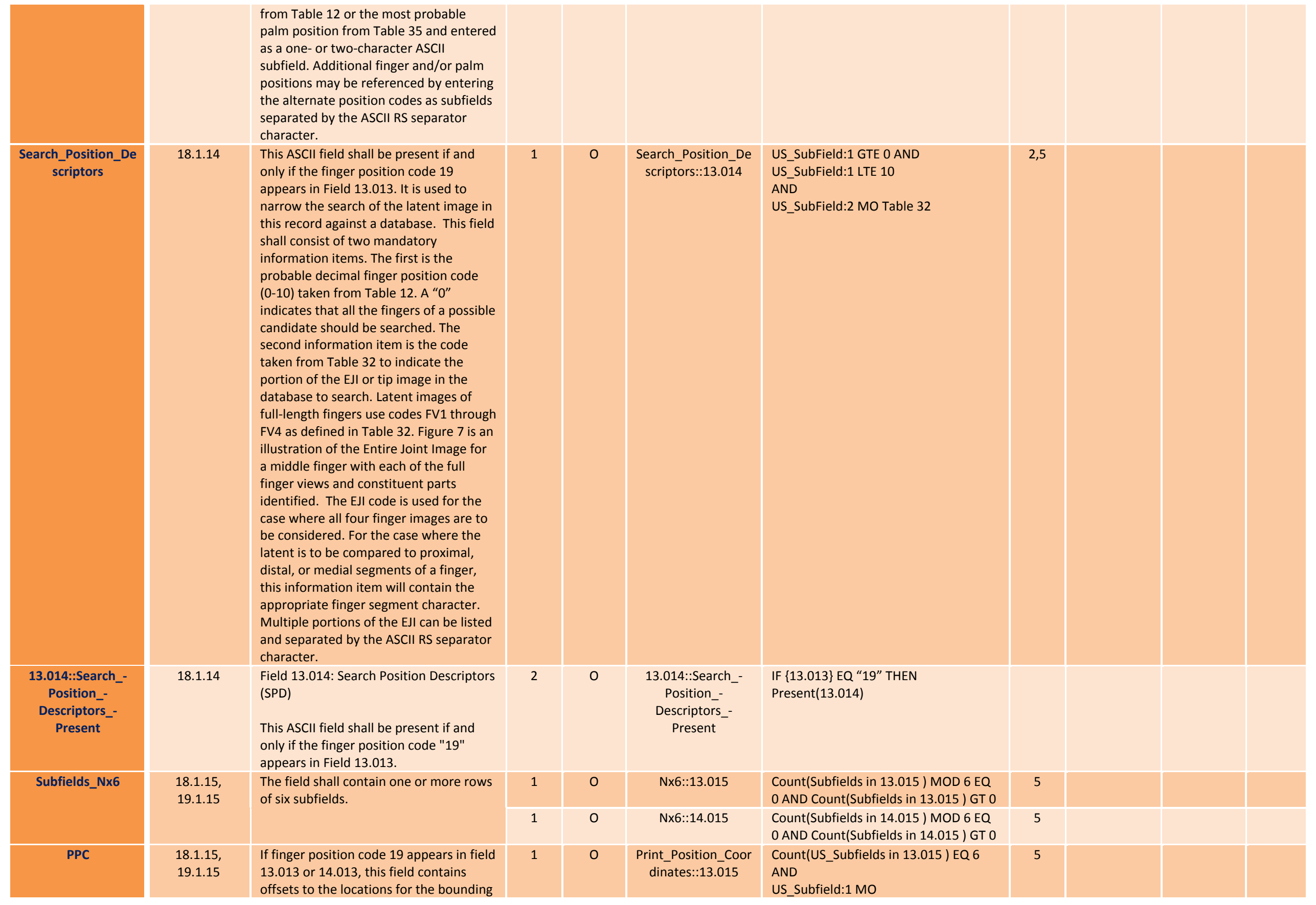




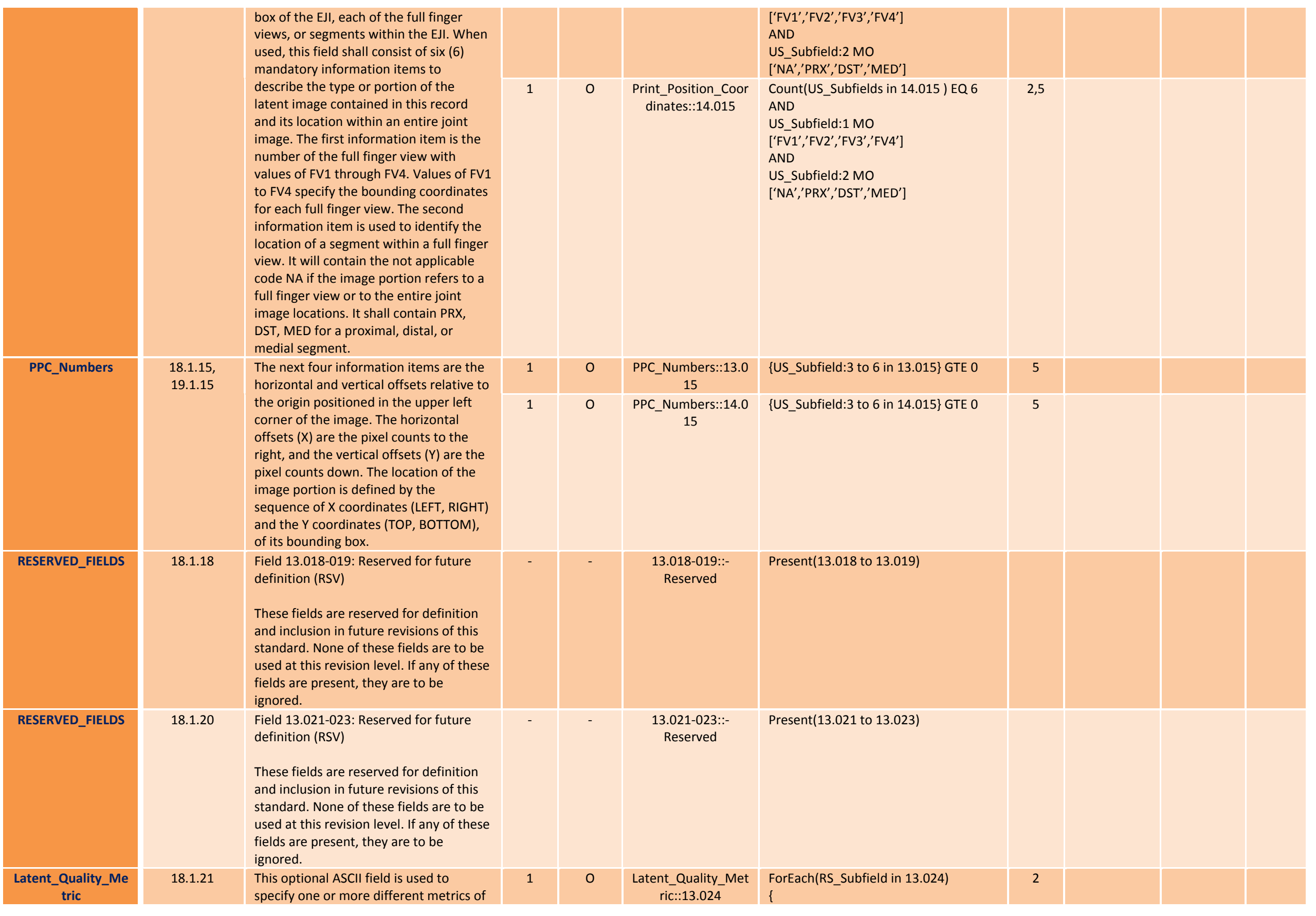


latent image quality score data for the image stored in this record. The

meaning attributed to this metric must

be defined and interpreted by the

producer of the scoring algorithm or by the person or system used to assign the metric to the latent image. The metric

may be a predictor of AFIS matcher

accuracy performance or a different

metric to indicate a value associated

with the quality of the latent image for a particular function.

This field may contain one or more subfields, each consisting of four

information items separated by the "US" separator character. The first

information item is the code as chosen from Table 12 or Table 35.

The other three items identify a quality score and the algorithm used to create the quality score. This information is useful to enable the recipient of the

quality score to differentiate between quality scores generated by different

algorithms and adjust for any

differences in processing or analysis as necessary.

The second information item shall be a quantitative expression of the predicted matching performance of the biometric sample. This item contains the ASCII representation of the integer image quality score between 0 and 100

assigned to the image data by a quality algorithm. Higher values indicate better quality. An entry of " 255 " shall indicate a failed attempt to calculate a quality score. An entry of " 254 " shall indicate that no attempt to calculate a quality score was made. The use of additional values to convey other information should be harmonized with ISO/IEC 19794 standards.

The third information item shall specify the ID of the vendor of the quality algorithm used to calculate the quality score. This 4-digit hex value is assigned by IBIA and expressed as four ASCII

\section{Count(US_Subfield) EQ 4}

AND

US_Subfield:1 MO Table12 or Table 35

AND

US Subfield:2 GTE 0 AND

US Subfield:2 LTE 100 OR

US_Subfield:2 MO [254,255]

AND

US Subfield:3 MO [IBIA Vendor Registry]

AND

US_Subfield:4 GTE 1 AND

US_Subfield:4 LTE 65535

\} 


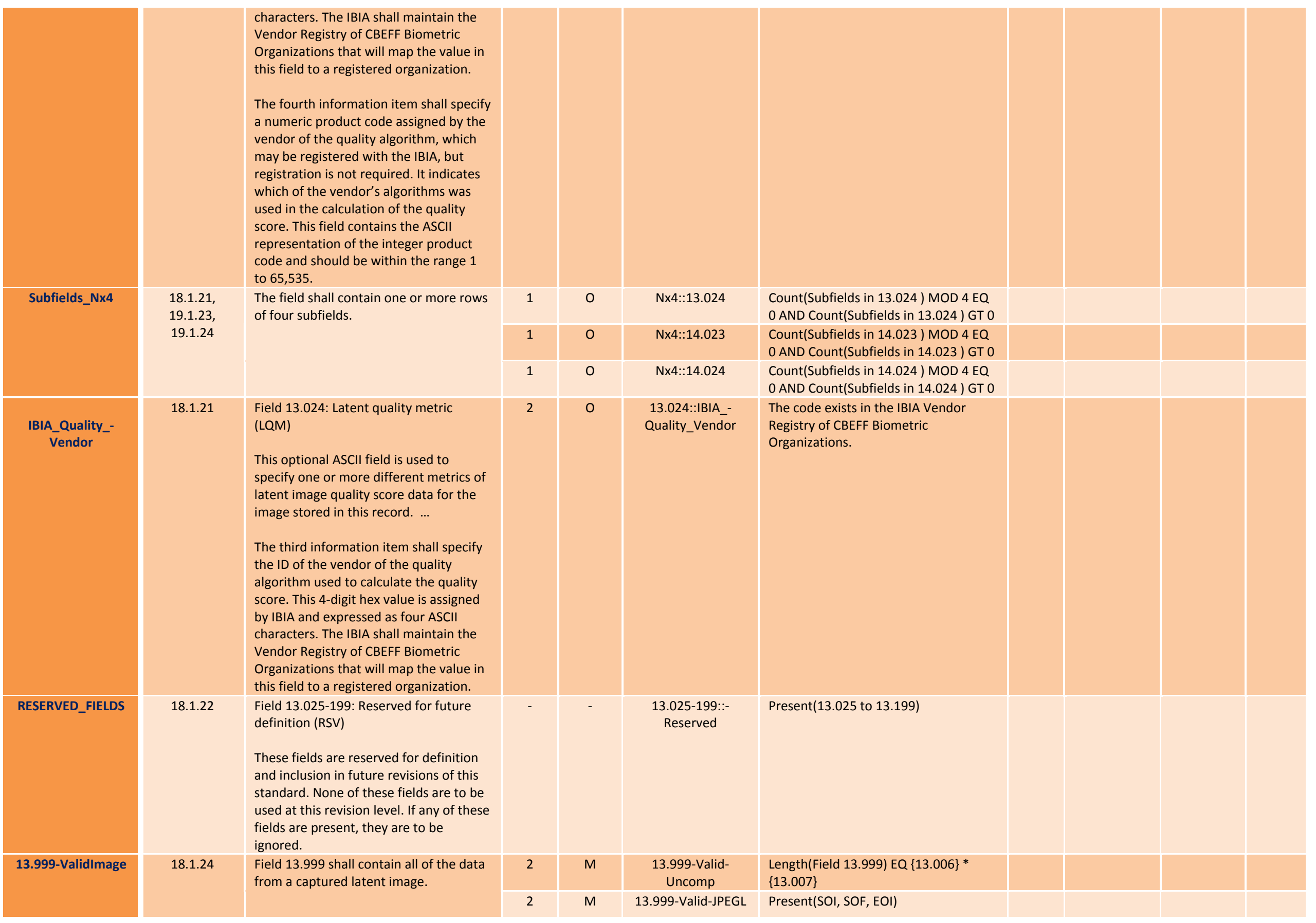




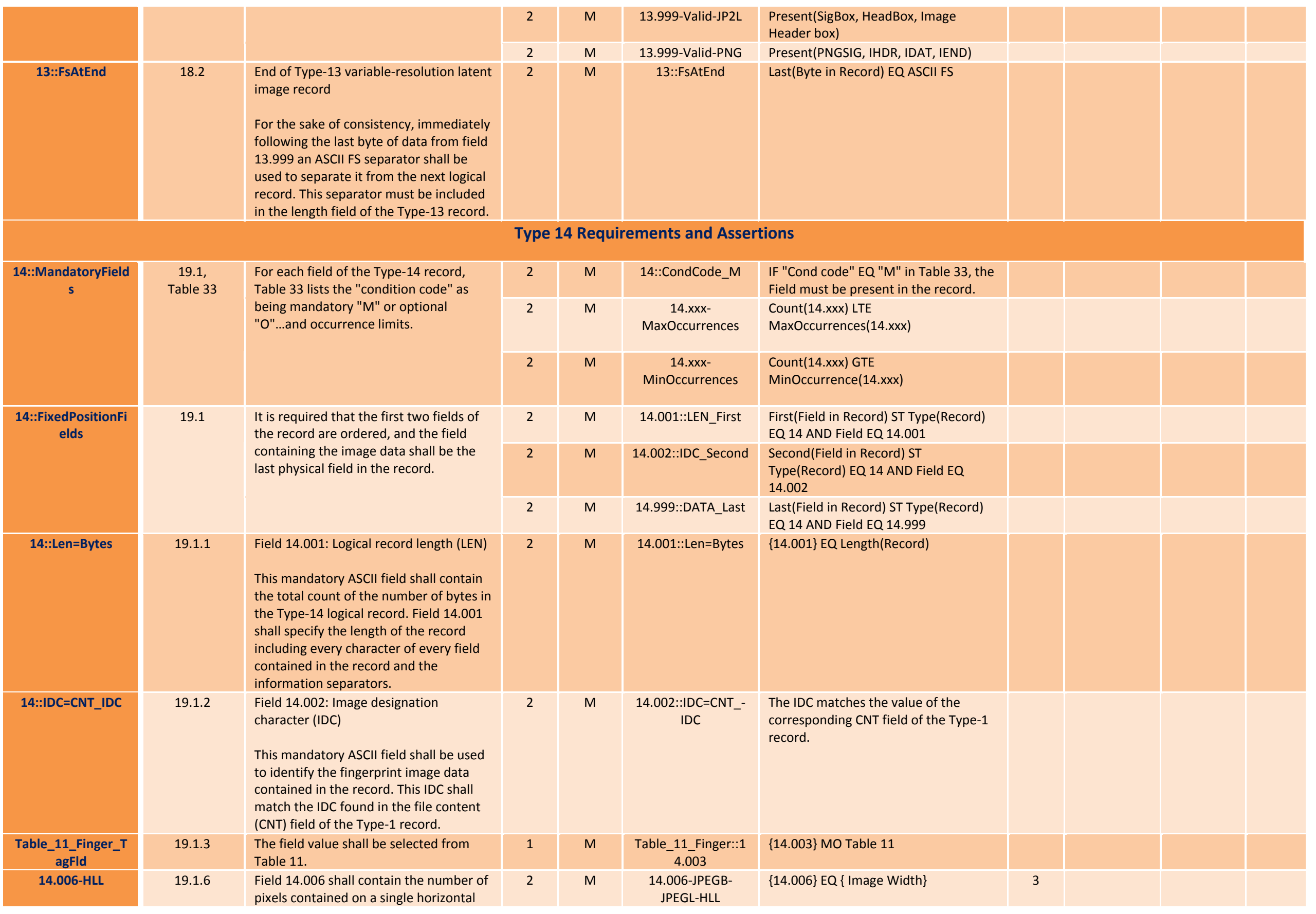




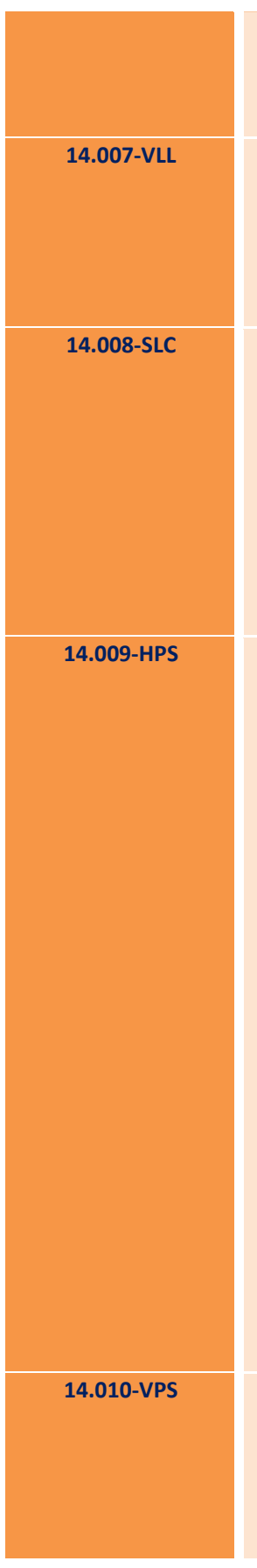

19.1.7 Field 14.007 shall contain the number of horizontal lines contained in the transmitted image.

Field 14.008 shall specify the units used to describe the image sampling frequency (pixel density).

Field 14.009 shall specify the integer pixel density used in the horizontal direction of the transmitted image if the SLC field contains a 1 or 2. Otherwise, it indicates the horizontal component of the pixel aspect ratio.

Field 14.010 shall specify the integer pixel density used in the vertical

direction of the transmitted image if the SLC field contains a 1 or 2. Otherwise, it indicates the vertical component of the pixel aspect ratio.

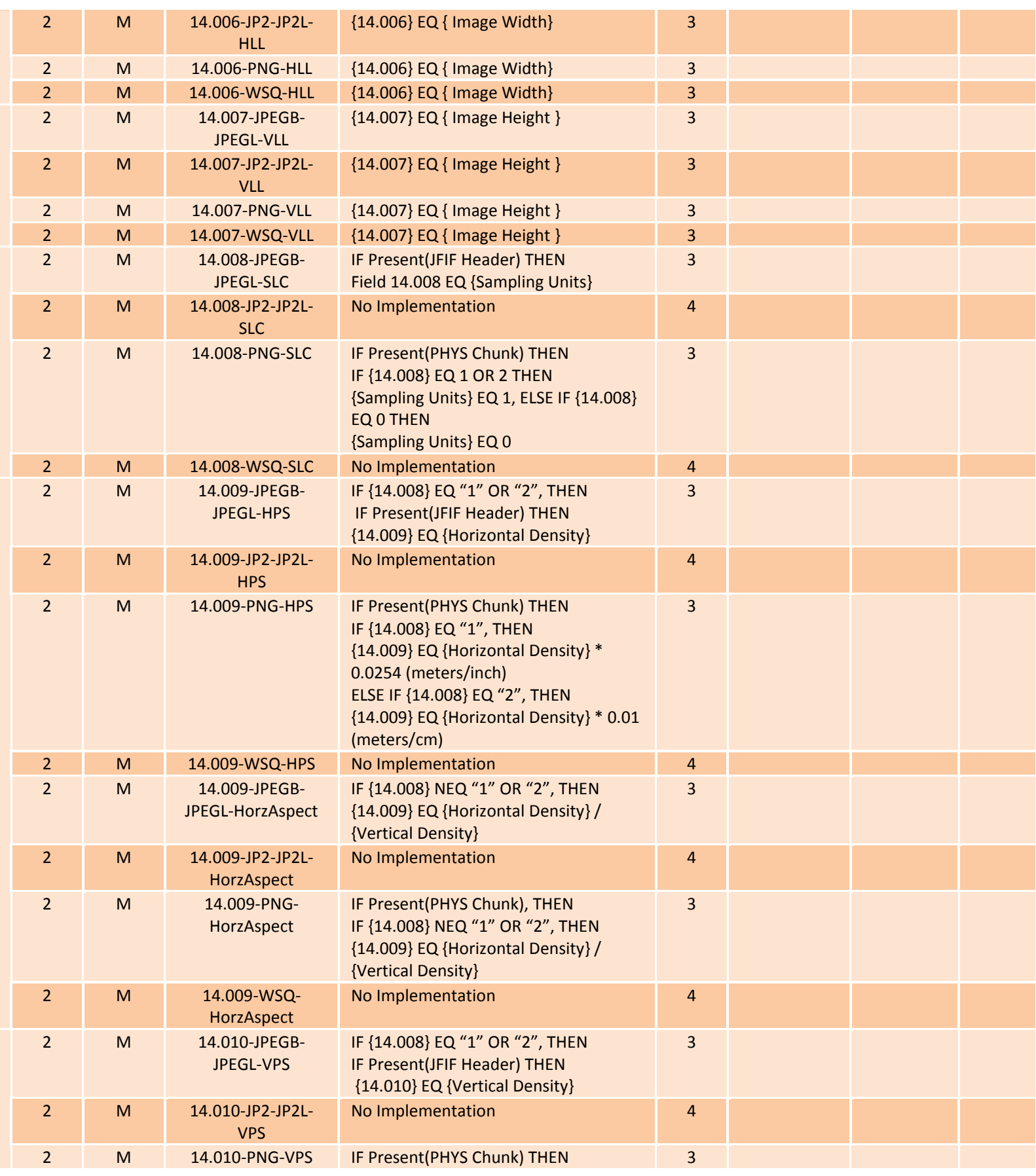




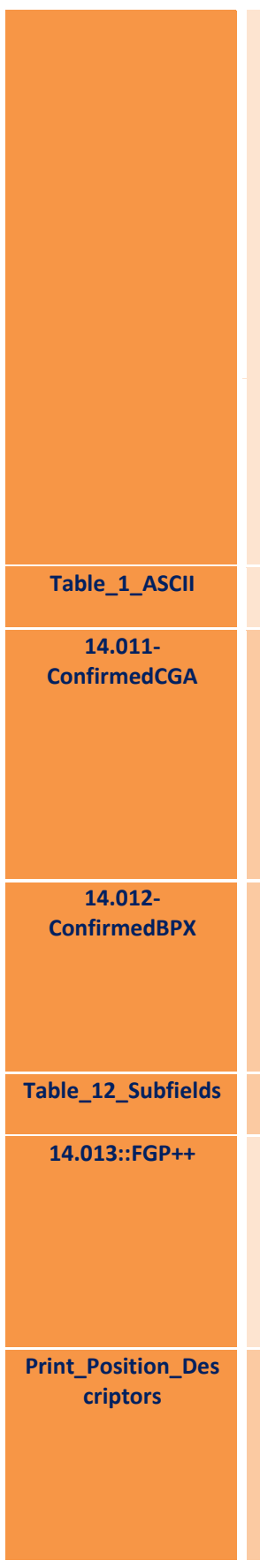

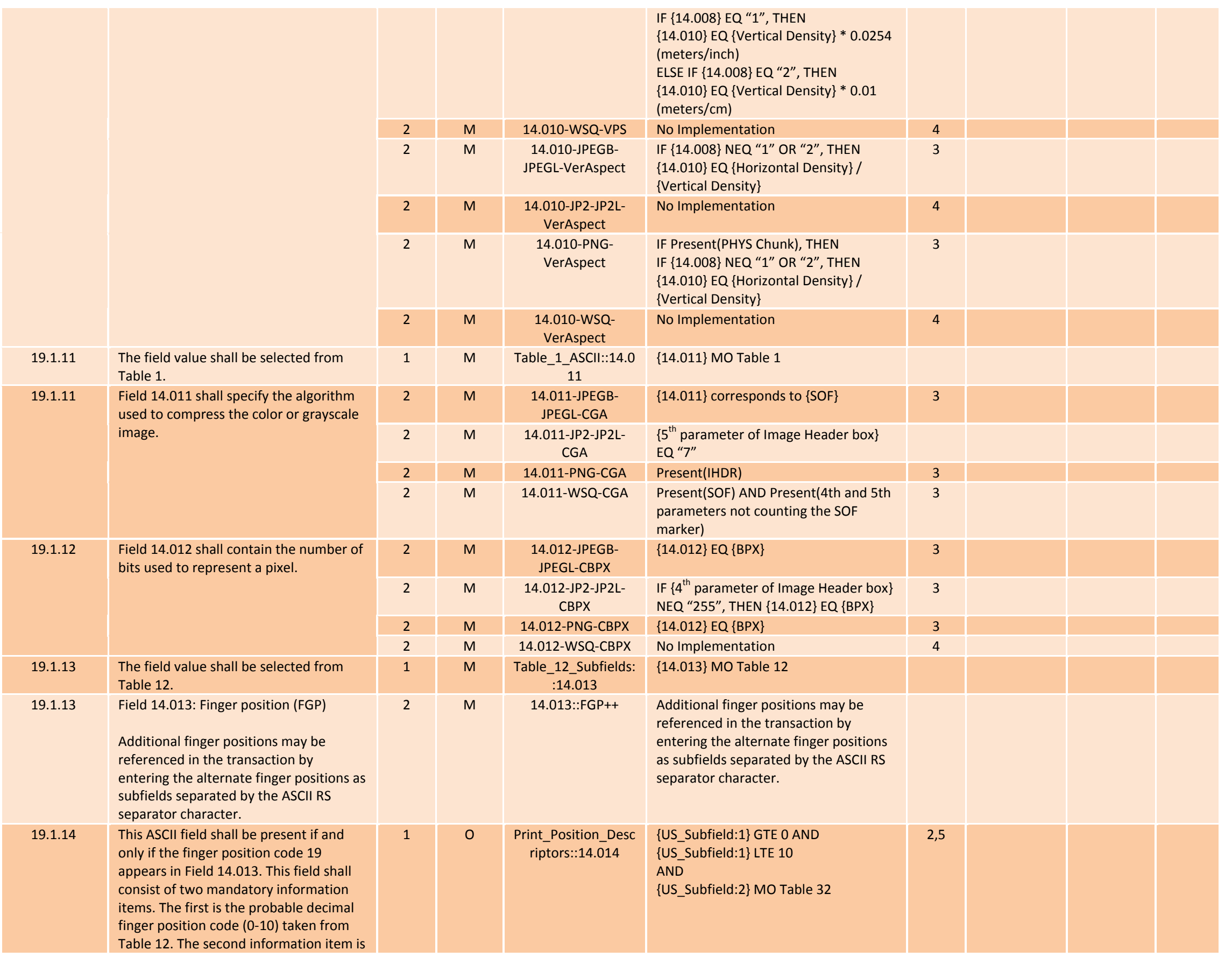




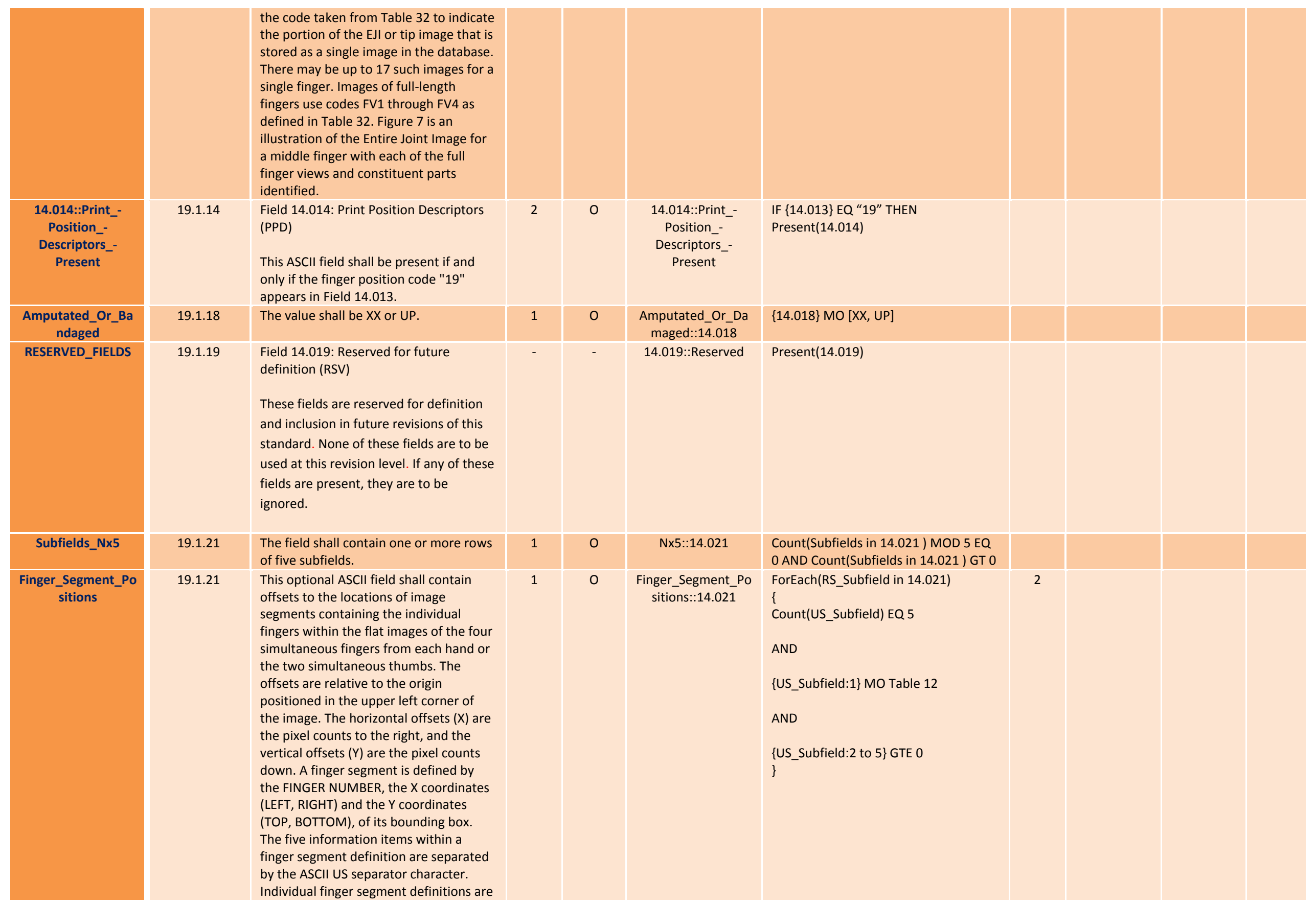






separated by the ASCII RS separator character.. If more than one algorithm is used to segment the image, successive sets finger segmentation positions shall be formatted as above and immediately follow the previous set.

19.1.22 This optional ASCII field shall contain the NIST Fingerprint Image Quality (NFIO) scores for the individual finger(s) derived from the slap impressions or individual rolled fingerprints. It consists of two information items. The first item is the finger number between one and ten as chosen from Table 12. The second item is the quality score which is a quantitative expression of the predicted AFIS matcher accuracy performance of the fingerprint image. The scores range from 1 for the best quality image, to 5 for the worst quality image. A 254 indicates that no score was ever computed while an entry of 255 shall indicate a failed attempt to calculate the image quality metric. These two information items are separated by the ASCII US separator character. Individua finger quality definitions are separated by the ASCII RS separator character. \begin{tabular}{l|l} 
19.1.23 & This optional ASCII field provides a \\
measure of estimated correctness
\end{tabular} regarding the accuracy of the location of the segmented finger within the right or left four finger or two thumbs slap image. For each segmented finger, this field shall contain four information items separated by the ASCII US separator character. The first information item is the finger number between one and ten as chosen from Table 12. The other three items identify a quality score and the algorithm used to create the quality score. This information is useful to enable the recipient of the quality score to differentiate between quality scores generated by different algorithms and adjust for any differences in processing or analysis as necessary. The second information item shall be a measure of estimated correctness regarding the accuracy of the location of the segmented finger. This item contains the ASCII representation of the integer

\begin{tabular}{|c|c|c|c|c|c|c|c|}
\hline 1 & 0 & NIST_Quality_Metri \\
c::14.022
\end{tabular}

Count(US_Subfield) EQ 4

AND

\{US_Subfield:1\} GTE 1 AND

\{US_Subfield:1\} LTE 10

AND

\{US_Subfield:2\} GTE 0 AND

\{US Subfield:2\} LTE 100 OR

\{US Subfield:2\} MO [254,255]

AND

\{US_Subfield:3\} MO [IBIA Vendor Registry]

AND

US Subfield:4\} GTE 1 AND

\{US_Subfield:4\} LTE 65535 OR 


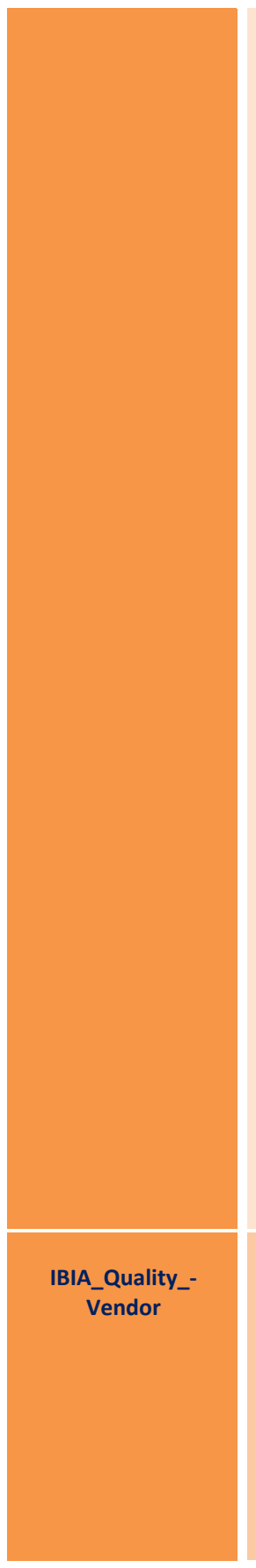

image quality score between 0 and 100 assigned to the image data by a quality algorithm. Higher values indicate better quality. An entry of 255 shall indicate a failed attempt to calculate a quality

score. An entry of 254 shall indicate that no attempt to calculate a quality score was made. The use of additional values to convey other information should be harmonized with ISO/IEC 19794

standards. The third information item shall specify the ID of the vendor of the quality algorithm used to calculate the quality score. This 4-digit hex value is assigned by IBIA and expressed as four ASCII characters. The IBIA shall maintain the Vendor Registry of CBEFF Biometric Organizations that will map the value in this field to a registered organization.

The fourth information item shall specify a numeric product code assigned by the vendor of the quality algorithm, which may be registered with the IBIA, but registration is not required. It indicates which of the vendor's algorithms was used in the calculation of the quality score. This field contains the ASCII

representation of the integer product code and should be within the range 1 to 65535 . This subfield is repeated for each segmented finger whose

coordinates appear in field 14.021. The ASCII RS separator character, separates each set of four information items. For the case where more than one

segmentation algorithm is applied to a

multi-finger plain image, the set of

segmentation information items for

each finger shall be ordered

corresponding to the entries in field

14.021.

19.1.23 Field 14.023: Segmentation quality metric (SQM)

The third information item shall specify the ID of the vendor of the quality algorithm used to calculate the quality score. This 4-digit hex value is assigned by IBIA and expressed as four ASCI characters. The IBIA shall maintain the Vendor Registry of CBEFF Biometric

Organizations that will map the value in

\begin{tabular}{|l|l|l|}
\hline 2 & 0 & $\begin{array}{l}14.023:: I B I A- \\
\text { Quality_Vendor }\end{array}$ \\
\hline
\end{tabular}




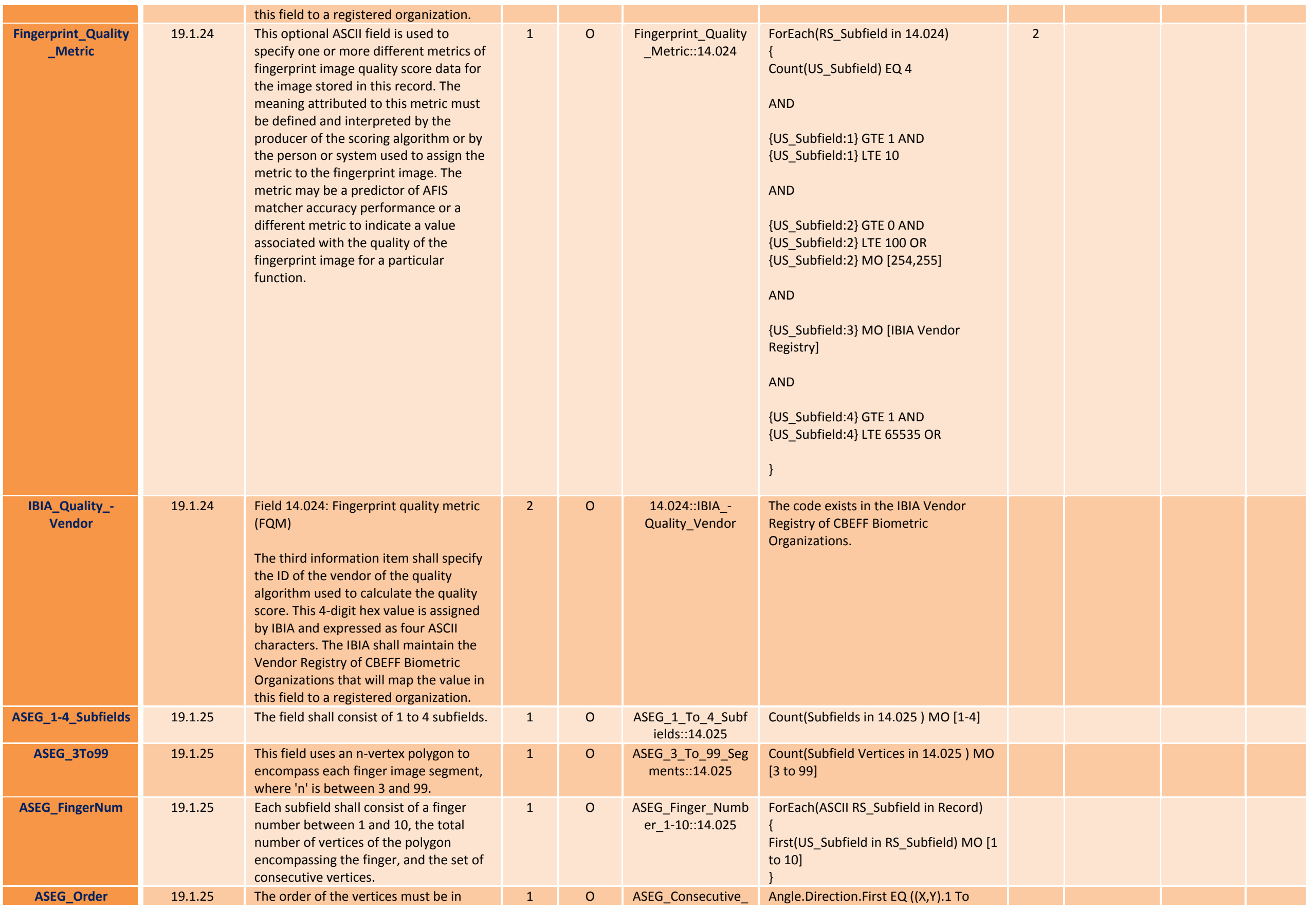




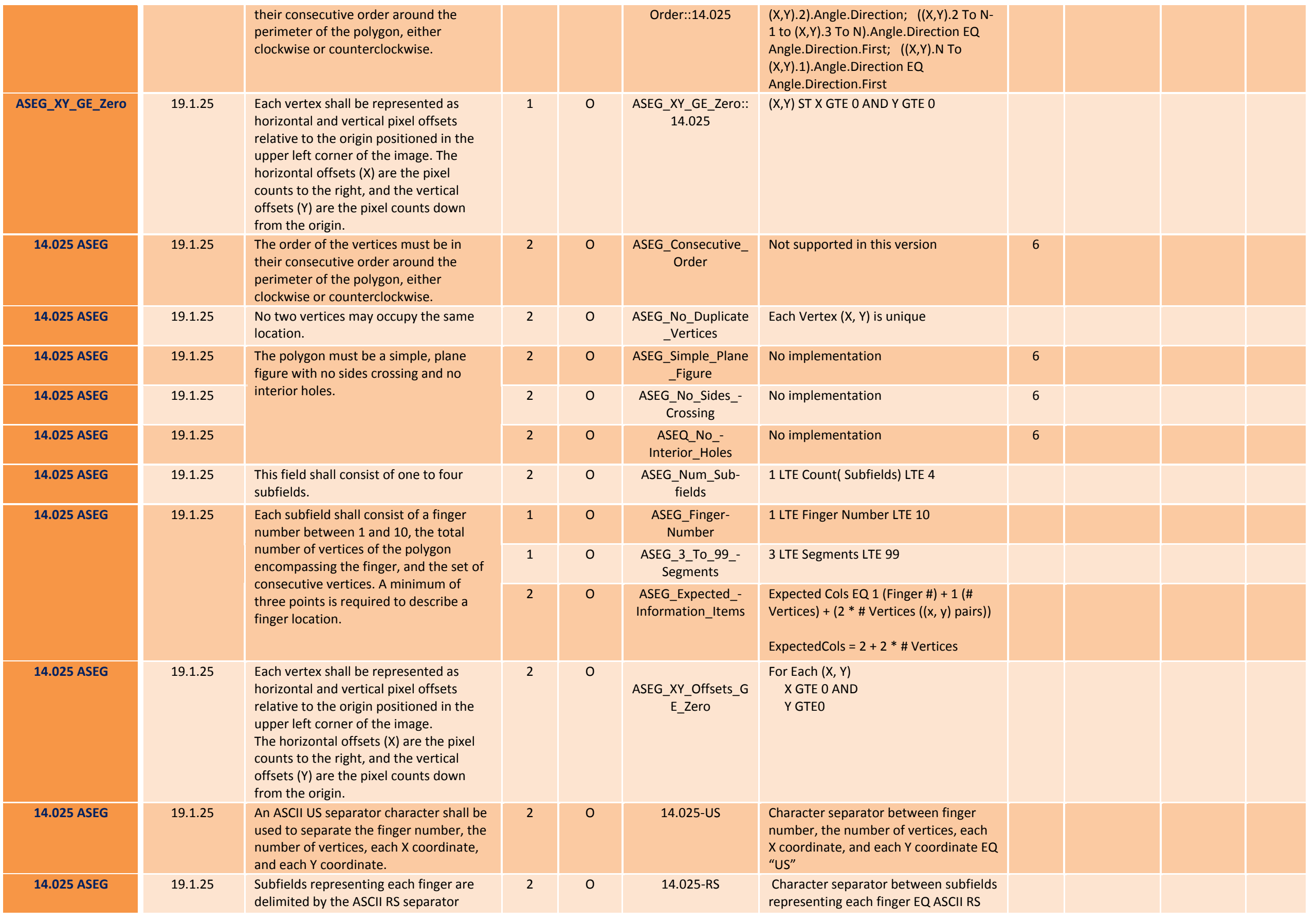




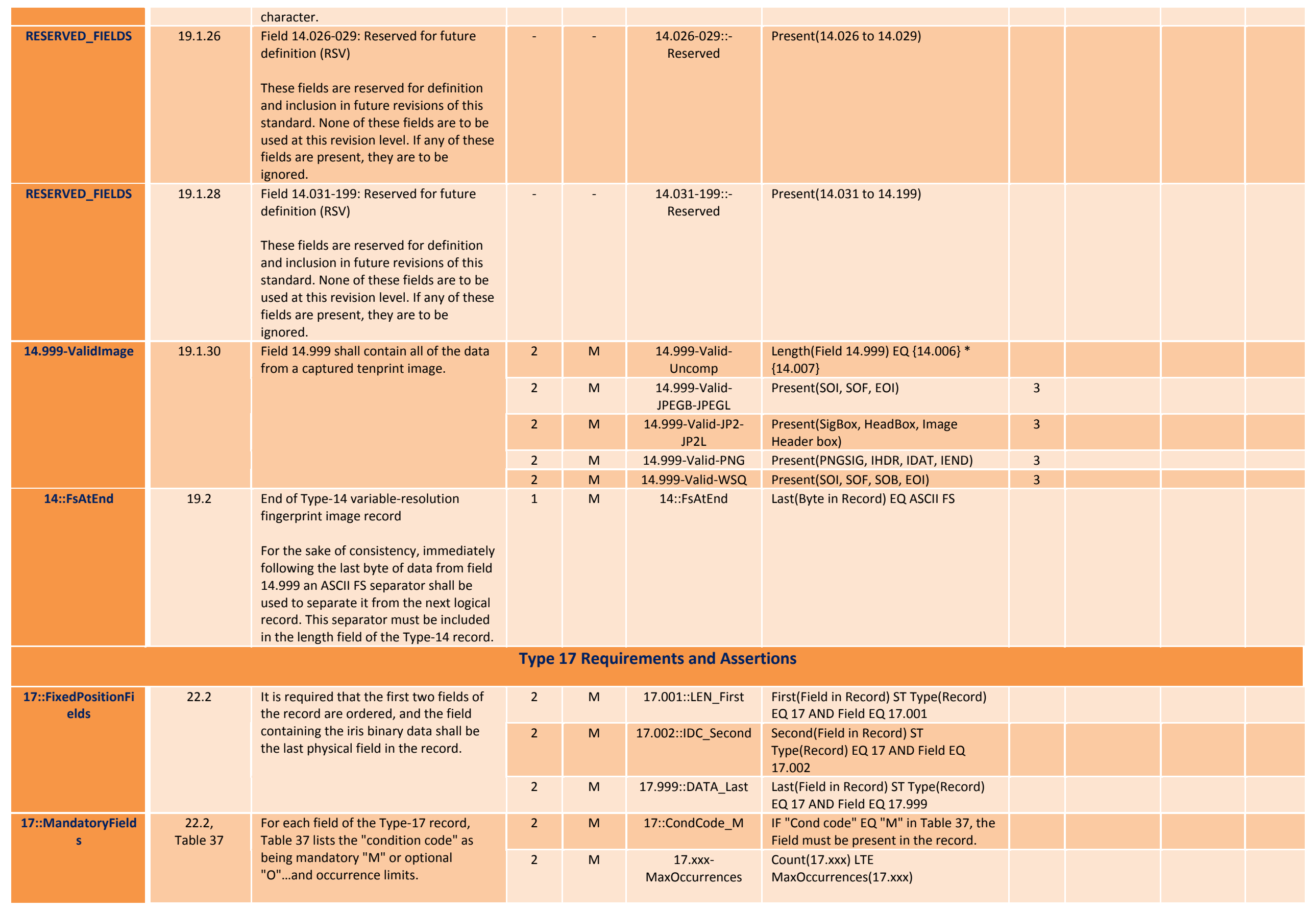




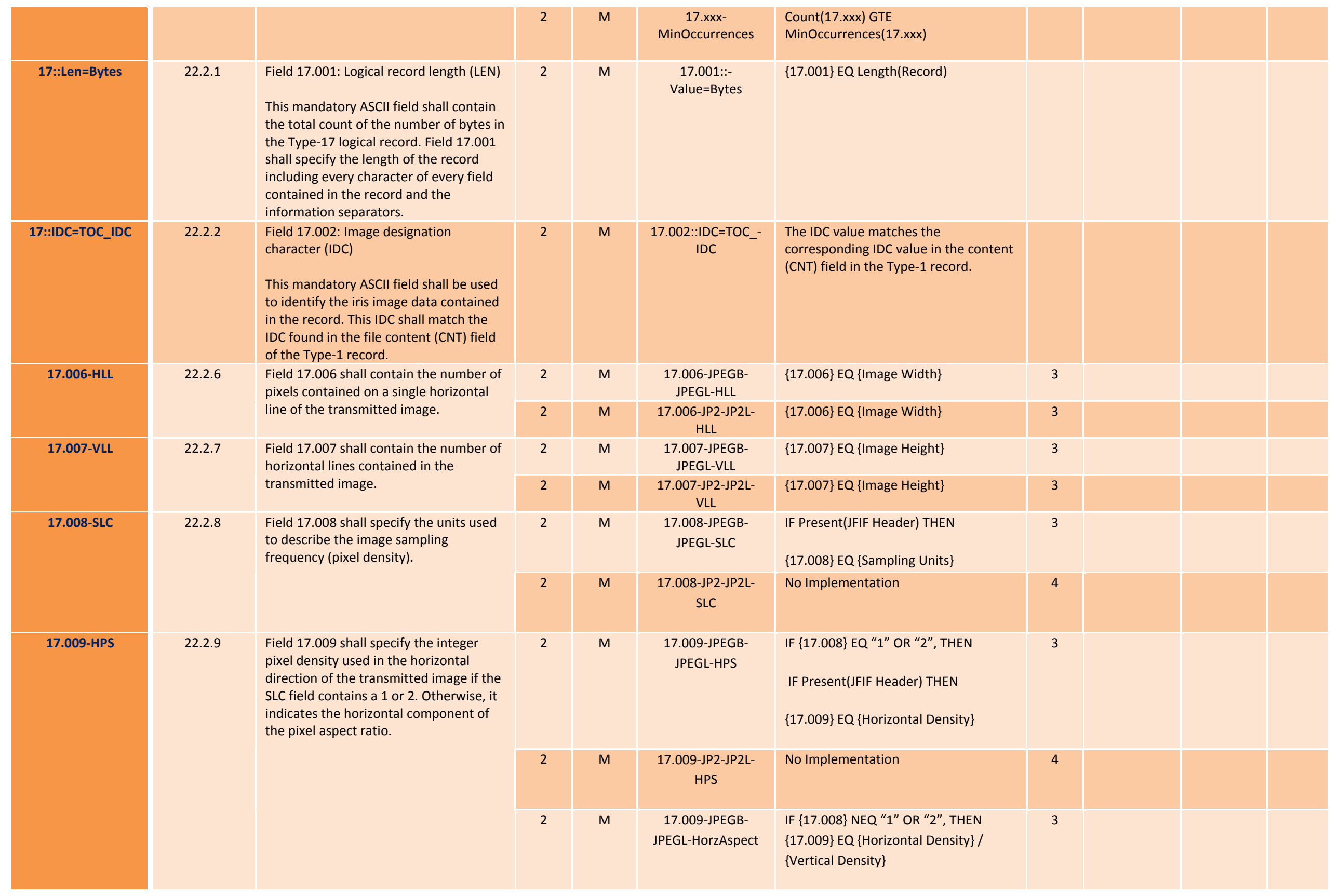




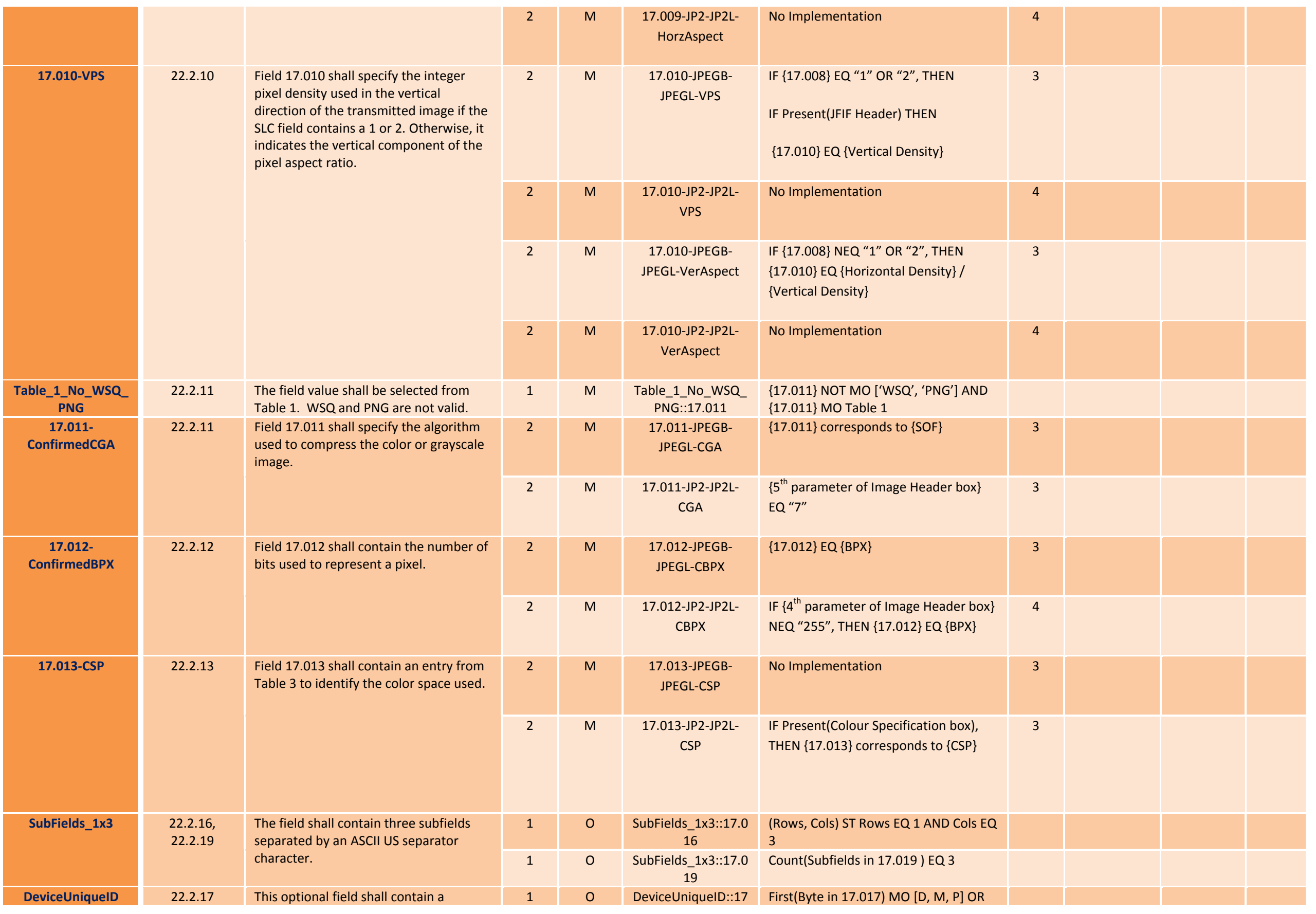




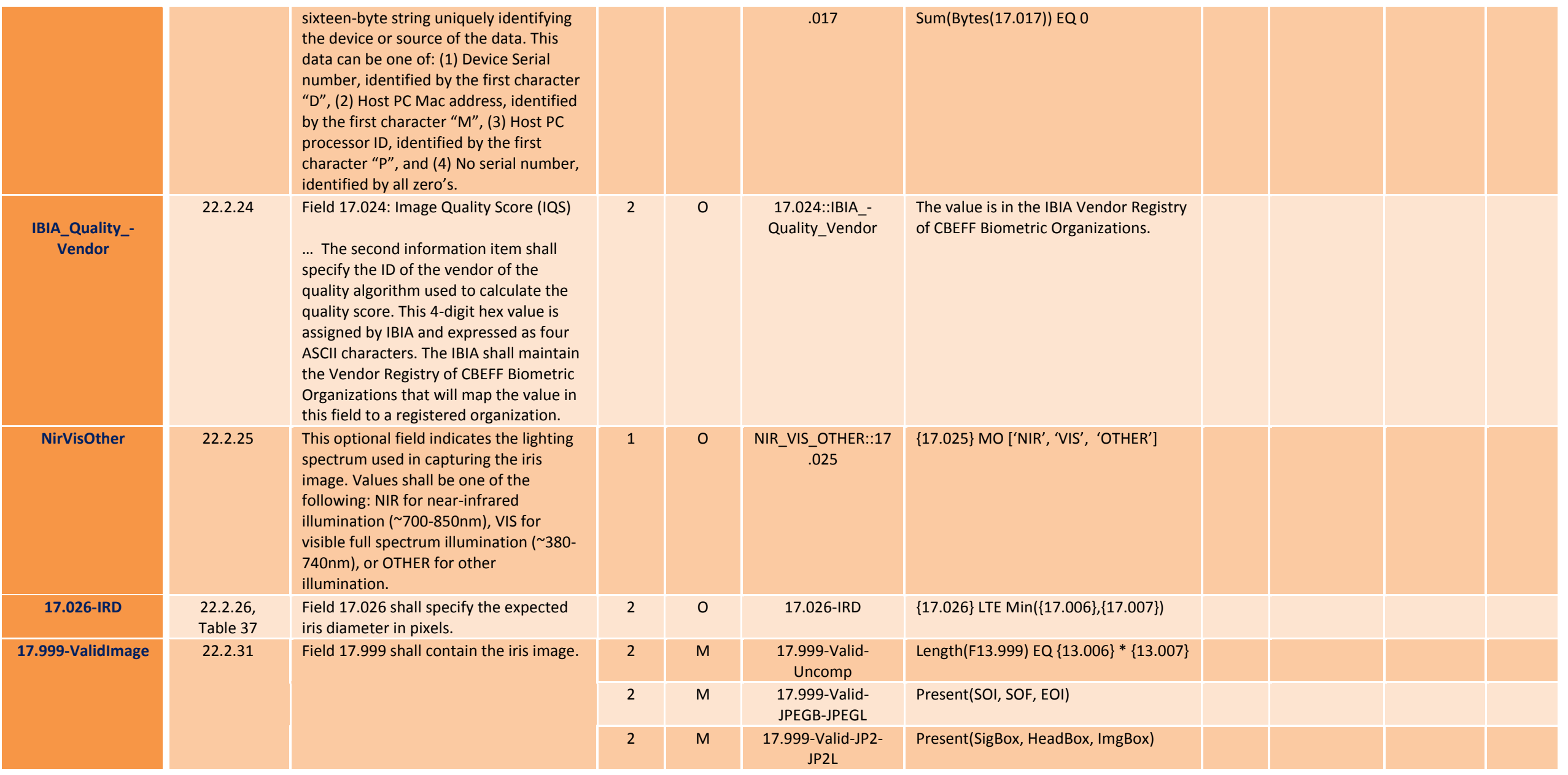

\section{Test Notes:}

1. Requirements related to International Character Sets are not tested in this version of the CTS.

2. Subfields are defined as follows:

- RS_Subfield:N= Any subfield that uses the ASCII RS separator character. If N is specified, N represents the Nth such subfield in the field

- US_Subfield:N = Any subfield that uses the ASCII US separator character. If N is specified, $N$ represents the Nth such subfield in the field

- $\quad$ Subfield(s) = If a prefix is not specified (US or RS), then the type of subfield should be clear from the context of the requirement. 
3. The table lists assertions for multiple image format types, however, based upon the compression algorithm specified in the record, specific image formats are to be tested in different ways. Features of the image metadata are defined as follows:

- Image Width

JPEG, JPEGL: $4^{\text {th }}$ parameter of the Frame Header not counting the SOF marker

JP2,JP2L: $2^{\text {nd }}$ parameter of Image Header box

○ PNG: $1^{\text {st }}$ parameter of IHDR chunk

WSQ: $5^{\text {th }}$ parameter of SOF not counting the SOF marker

- Image Height

JPEG, JPEGL: $3^{\text {rd }}$ parameter of the Frame Header not counting the SOF marker

JP2,JP2L: $1^{\text {st }}$ parameter of Image Header box

PNG: $2^{\text {nd }}$ parameter of IHDR chunk

WSQ: $4^{\text {th }}$ parameter of SOF not counting the SOF marker

- Image metadata markers

- JPEG, JPEGL: SOI - Start of Image, SOF - Start of Frame, EOI - End of Image

○ JP2, JP2L: SigBox - JP2 Signature box, HeadBox - JP2 Header box, ImgBox - Image Header box

○ PNG: PNGSig - PNG Signature, IHDR - IHDR Chunk, IDAT - IDAT Chunk, IEND - IEND Chunk

- WSQ: SOI - Start of Image, SOF - Start of Frame, SOB - Start of Block, EOI - End of Image

- Sampling Units

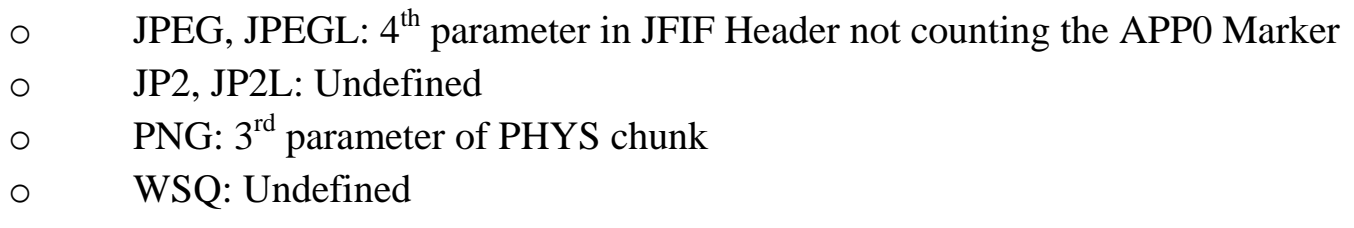

- Horizontal Density

O JPEG, JPEGL: $5^{\text {th }}$ parameter in JFIF Header not counting the APP0 Marker

J JP2, JP2L: Undefined

P PNG: $1^{\text {st }}$ parameter in PHYS Chunk

○ WSQ: Undefined

- Vertical Density
○ JPEG, JPEGL: $6^{\text {th }}$ parameter in JFIF Header not counting the APP0 Marker
$\circ \quad$ JP2, JP2L: Undefined
$\circ \quad$ PNG: $2^{\text {nd }}$ parameter in PHYS Chunk
$\circ$ WSQ: Undefined

- BPX 
JPEG, JPEGL: 2nd parameter of the Frame Header not counting the SOF marker

- JP2, JP2L: 7 LSB of $4^{\text {th }}$ parameter of Image Header box +1

- PNG: $3^{\text {rd }}$ parameter of IHDR chunk

- WSQ: Undefined

- CSP

\section{JPEG,JPEGL: Undefined}

- JP2, JP2L: $4^{\text {th }}$ parameter of Colour Specification box

- PNG: $4^{\text {th }}$ parameter of IHDR chunk

$\circ \quad$ WSQ: Undefined

4. The image metadata required to implement the assertion is not defined in the image standard documentation.

5. While the ANSI/NIST-ITL 1-2007 standard defines only the "M" and "O" statuses for each field, this field is conditional upon the content of the other field(s).

6. The required algorithm for this assertion is under research. 